\title{
Dirac Equation with External Potential AND Initial Data ON CAUChy SuRfaCES
}

\author{
D.-A. Deckert \\ Mathematical Department of the University of California Davis \\ One Shield Ave, CA 95616 Davis, USA \\ deckert@math.ucdavis.edu \\ F. Merkl \\ Mathematisches Institut der Ludwig-Maximilians-Universität München \\ Theresienstr. 39, 80333 München, Germany \\ merkl@mathematik.uni-muenchen.de
}

April 8, 2014

\begin{abstract}
With this paper we provide a mathematical review on the initial-value problem of the one-particle Dirac equation on space-like Cauchy hypersurfaces for compactly supported external potentials. We, first, discuss the physically relevant spaces of solutions and initial values in position and mass shell representation; second, review the action of the Poincaré group as well as gauge transformations on those spaces; third, introduce generalized Fourier transforms between those spaces and prove convenient Paley-Wiener- and Sobolev-type estimates. These generalized Fourier transforms immediately allow the construction of a unitary evolution operator for the free Dirac equation between the Hilbert spaces of square-integrable wave functions of two respective Cauchy surfaces. With a Picard-Lindelöf argument this evolution map is generalized to the Dirac evolution including the external potential. For the latter we introduce a convenient interaction picture on Cauchy surfaces. These tools immediately provide another proof of the well-known existence and uniqueness of classical solutions and their causal structure.
\end{abstract}

\section{Contents}

1 Introduction and Motivation 2

2 The One-Particle Dirac Equation $\quad 4$

2.1 Relevant Spaces in Space-Time Representation . . . . . . . . . . . . . . . . 5 
2.2 Relevant Spaces in Energy-Momentum Representation . . . . . . . . . . . . 7

2.3 Action of the Poincaré Group ． . . . . . . . . . . . . . . . . . . 10

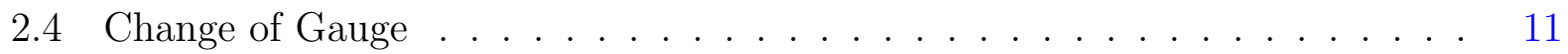

2.5 Generalized Fourier Transforms . . . . . . . . . . . . . . . . . . . . 12

2.5.1 Paley-Wiener Bounds . . . . . . . . . . . . . . . 13

2.5.2 Sobolev Norms . . . . . . . . . . . . . . . . . . . . 14

2.6 Existence, Uniqueness, and Causal Structure . . . . . . . . . . . . . 15

2.7 An Interaction Picture on Cauchy Surfaces . . . . . . . . . . . . . . . . . 16

$\begin{array}{llr} & \text { Proofs } & 18\end{array}$

3.1 Generalized Fourier Transforms . . . . . . . . . . . . . . . 18

3.1.1 Properties of the Maps $\mathcal{F}_{3 \mathcal{M}}$ and $\mathcal{F}_{\mathcal{M} 3} \ldots \ldots \ldots \ldots$

3.1.2 Properties of the Maps $\mathcal{F}_{\mathcal{M} \Sigma}, \mathcal{F}_{\Sigma 0}$ and $\mathcal{F}_{0 \mathcal{M}} \ldots \ldots \ldots$. . . . . . . . . . . . . . . . . . . .

3.1.3 Proof of Theorem $2.14 \ldots \ldots \ldots$. . . . . . . . . . . . . . 31

3.2 Existence, Uniqueness, and Causal Structure . . . . . . . . . . . . . . . 31

3.2.1 Existence and Uniqueness in the Interaction Picture . . . . . . . . . . 31

3.2.2 Regularity and Support Properties . . . . . . . . . . . . . . . 34

3.2.3 Proof of Theorem 2.23 . . . . . . . . . . . . . . . . . . . . . . . . . . . . . . . . . 35

3.2 .4 Proof of Theorem 2.20 . . . . . . . . . . . . . . . . . . . 36

3.2.5 Proof of Theorem $2.22 \ldots \ldots \ldots \ldots$

$\begin{array}{ll}\text { A Auxiliary Results } & 37\end{array}$

\section{Introduction and Motivation}

The one-particle Dirac equation plays a fundamental role in relativistic quantum theory. It was introduced by Dirac to describe the dynamics of spin $1 / 2$ fermions such as electrons. Although as a one-particle equation alone its physical interpretation is difficult due to the occurrence of negative energy states, its second-quantized form leads to the so-called external field or no-photon quantum electrodynamics. In this respect, our interest in the solution theory for initial data on space-like hypersurfaces is three-fold:

(1) While a mathematical rigorous construction of the second-quantized time evolution in a fixed fermionic Fock space has only been carried out successfully in the case of zero spacelike components of the external four-vector potential, physicists have developed powerful recipes to extract predictions in terms of formal perturbation series from external field quantum electrodynamics despite the ill-defined nature of its equations of motion; e.g., see [Dys06, Sch61] for an overview. Whether the resulting series do converge or in which regimes the corresponding corrections are small seems to be unknown. This fact becomes particular unsatisfactory in light of next generation laser experiments such as planned to be conducted, e.g., at the Extreme Light Infrastructure [ELI]. These will allow to probe quantum electrodynamics in strong-field regimes in which, first, the conventional perturbative techniques become questionable, and second, a mere scattering theoretic description of physical processes is not sufficient and a dynamical description is needed; cf. [Dun09]. 
The obstacle in the construction of a second-quantized time evolution was observed in the works [SS65, Rui77a, Rui77b]. There it is shown that the one-particle time evolution can be lifted to the Fock space if and only if the space-like components of the external four-vector potential are zero. One way out of this dilemma, as sketched in [FS79], is to implement the time evolution on time-varying Fock spaces. Two different such constructions have been carried out in [LM96, Mic98] and [DDMS10]. Both involve additional degrees of freedom (such as the charge-renormalization) which can be encoded in the choice of a phase depending on the external field; see [DDMS10] for the identification of the dependence of these degrees of freedom on the external field. The resulting second-quantized time evolution transports initial data from one equal-time hyperplane to another and gives rise to unique transition probabilities. However, quantities such as the charge-current density depend manifestly on this unidentified phase. In particular, this concerns the so-called phenomenon of vacuum polarization but also the dynamical description of pair creation processes for which so far only a few rigorous treatments are available; see [GHLS13] for vacuum polarization in the Hartree-Fock approximation for static external sources and [DP07] for adiabatic pair creation. The involved degrees of freedom can be reduced further by imposing the Bogolyubov causality condition [BS59, (17.30)] or more or less equivalently by implementing second-quantized evolution maps between Fock spaces associated to space-like Cauchy hypersurfaces, which is the content of a follow-up work. During our study of the latter approach a detailed knowledge of the one-particle Dirac equation for initial data on space-like hypersurfaces proved to be essential. Collecting this knowledge is our main motivation for writing this paper.

Another possible way out of the mentioned dilemma that deserves mentioning lies in a reformulation of quantum electrodynamics in terms of the so-called fermionic projector for which we refer the reader to [Fin06].

Apart from this we have two more general interests:

(2) As well as classical electrodynamics also quantum electrodynamics is a manifestly Lorentz covariant theory. However, its Lorentz covariance is often obscured in the presentation of the theory when its fundamental equations of motion are formulated exclusively on equal-time hyperplanes. Non-trivial Lorentz boosts, however, tilt any equal-time hyperplane in space-time and, therefore, Lorentz covariance of quantum electrodynamics is only apparent in the momentum representation of the free theory or in the asymptotic description of the corresponding scattering theory. In order to make Lorentz and gauge covariance explicit in a space-time representation we provide necessary mathematical results for the Dirac equation that allow to work exclusively with initial data on spacelike Cauchy hypersurfaces in the spirit of Tomonaga and Schwinger [Tom46, Sch48]. In this regard our efforts are intended to contribute towards a mathematically rigorous understanding of their works.

(3) Furthermore, by deforming a Cauchy surface in a small neighborhood of a point, the dynamics can be studied locally; compare the differential formulation of the equations of motion (25) in [Tom46]. Providing mathematical tools for such a study is our third motivation for this paper. One important observation is that due to the causal structure 
of solutions of the Dirac equation only local information about the external potential is needed. In consequence, the behavior of the external potential and of the Cauchy surfaces near "infinity" is irrelevant for finite times. This justifies the technical convenience of restricting our study to compactly supported external potentials.

There are several treatises of the Dirac equation in the classical literature which discuss the Dirac equation on equal-time hyperplanes, most prominently [Tha92]. The initial value problems for such hyperbolic systems of differential equations was already treated in, e.g., [Joh82, Tay11]. Wave equations on Lorentzian manifolds including the Dirac equation have been studied, e.g., in [Dim82], [BGP07], [Rin09], [FKT12], and [DG13]. In particular, their results ensure existence and uniqueness of solutions and identify their causal structure. The main contribution of our work is the introduction of general Fourier transform and corresponding Paley-Wiener techniques that can be exploited in flat space-time to study solutions to the one-particle Dirac equation on Cauchy surfaces. A byproduct of these generalized Fourier transform methods yields yet another proof for the well-posedness of the initial value problem of Dirac equation on Cauchy surfaces. Therefore we used this opportunity to compile our results in the form of a self-contained review given in Section 2 that ranges from general assertions about the initial value problem to a detailed analysis of solutions. To increase readability the more technical proofs are provided separately in Section 3.

Acknowledgment. The authors cordially thank Wojciech Dybalski and Felix Finster for their helpful and detailed suggestions on the classical and contemporary literature of the initial value problem of hyperbolic systems of differential equations.

Notation. Positive constants are denoted by $C_{1}, C_{2}, C_{3}, \ldots$ They keep their value throughout the whole article. Any fixed quantity a constant depends on is displayed at least once when the constant is introduced.

\section{The One-Particle Dirac Equation}

The one-particle Dirac equation for an electron of mass $m>0$ is given by

$$
(i \not \supset-A) \psi=m \psi
$$

where the external potential

$$
A=\left(A_{\mu}\right)_{\mu=0,1,2,3} \in \mathcal{C}_{c}^{\infty}\left(\mathbb{R}^{4}, \mathbb{R}^{4}\right)
$$

is assumed to be smooth and compactly supported. In our notation the elementary charge $e$ (having a negative sign in the case of an electron) is already included in $A$ and units are chosen such that $\hbar=1$ and $c=1$. Moreover, the elements of $\mathbb{R}^{4}$ are represented by $x=\left(x^{0}, x^{1}, x^{2}, x^{3}\right)=\left(x^{0}, \mathbf{x}\right)=x^{\mu} e_{\mu}$, where $e_{\mu}$ denotes the canonical basis vectors in $\mathbb{R}^{4}$. We endow $\mathbb{R}^{4}$ with the metric tensor $g=\left(g_{\mu \nu}\right)_{\mu, \nu=0,1,2,3}=\operatorname{diag}(1,-1,-1,-1)$. Raising and lowering indices is done w.r.t. this metric tensor. We employ Einstein's summation convention, 
Feynman's slash-notation $\not=\gamma^{\mu} \partial_{\mu}, A=\gamma^{\mu} A_{\mu}$, and use the standard representation of the Dirac matrices $\gamma^{\mu} \in \mathbb{C}^{4 \times 4}$ that fulfill $\left\{\gamma^{\mu}, \gamma^{\nu}\right\}=2 g^{\mu \nu}$.

Before touching the question about existence of solutions to the Dirac equation (1) in Section 2.6 we introduce and study the physically relevant classes of solutions and initial data in space-time and energy-momentum representation.

\subsection{Relevant Spaces in Space-Time Representation}

We now define the classes of possible solutions to the Dirac equation (1) and initial data in space-time representation considered in this work.

Definition 2.1 (Classical Solutions in Space-Time Representation). Let $\mathcal{C}_{A}$ denote the space of all smooth solutions $\psi \in C^{\infty}\left(\mathbb{R}^{4}, \mathbb{C}^{4}\right)$ of the Dirac equation (1) which have a spatially compact causal support in the following sense: There is a compact set $K \subset \mathbb{R}^{4}$ such that

$$
\operatorname{supp} \psi \subseteq K+\text { Causal, }
$$

where Causal $:=\left\{x \in \mathbb{R}^{4} \mid x_{\mu} x^{\mu} \geqslant 0\right\}$ denotes the set of all causal vectors.

One way to build a solution theory for the Dirac equation (1) is to generate solutions in $\mathcal{C}_{A}$ from initial data prescribed on Cauchy surfaces which, for our purposes, are defined as follows:

Definition 2.2 (Cauchy Surfaces). We define a Cauchy surface $\Sigma$ in $\mathbb{R}^{4}$ to be a smooth, 3-dimensional submanifold of $\mathbb{R}^{4}$ that fulfills the following three conditions:

(a) Every inextensible, two-sided, time-or light-like, continuous path in $\mathbb{R}^{4}$ intersects $\Sigma$ in a unique point.

(b) For every $x \in \Sigma$, the tangential space $T_{x} \Sigma$ is space-like.

(c) The tangential spaces to $\Sigma$ are bounded away from light-like directions in the following sense: The only light-like accumulation point of $\bigcup_{x \in \Sigma} T_{x} \Sigma$ is zero.

The differences compared to, e.g., the definition given in [Wal84, Section 8.3], are the smoothness condition as well as (b) and (c). Condition (c) is not essential, but convenient to use as we are mainly interested in the local and causal properties of the Dirac evolution.

Remark 2.3. The simplest Cauchy surface is the time-zero hyperplane

$$
\Sigma^{0}:=\left\{x \in \mathbb{R}^{4} \mid x^{0}=0\right\}
$$

Note that by definition, light-like vectors are not allowed as tangential vectors to $\Sigma$. For example

$$
\left\{\left(\arctan x^{1}, \mathbf{x}\right) \mid \mathbf{x}=\left(x^{1}, x^{2}, x^{3}\right) \in \mathbb{R}^{3}\right\}
$$

is not a Cauchy surface according to our definition, as condition (b) is violated. Moreover,

$$
\left\{\left(\sqrt{1+\mathbf{x}^{2}}, \mathbf{x}\right) \mid \mathbf{x}=\left(x^{1}, x^{2}, x^{3}\right) \in \mathbb{R}^{3}\right\}
$$


is not a Cauchy surface either, as condition (a) is violated. In coordinates, every Cauchy surface $\Sigma$ can be parametrized as

$$
\Sigma=\left\{\left(t_{\Sigma}(\mathbf{x}), \mathbf{x}\right): \mathbf{x} \in \mathbb{R}^{3}\right\}
$$

with a smooth function $t_{\Sigma}: \mathbb{R}^{3} \rightarrow \mathbb{R}$ that fulfills $\left|\nabla t_{\Sigma}(\mathbf{x})\right|<1$ for every $\mathbf{x} \in \mathbb{R}^{3}$. Note that this condition is necessary but not sufficient for $\Sigma$ satisfying conditions (a) and (b). This is illustrated by the second counterexample above. Condition (c) guarantees that even $\sup _{\mathbf{x} \in \mathbb{R}^{3}}\left|\nabla t_{\Sigma}(\mathbf{x})\right|<1$ holds.

In order to define the spaces of initial data it will be convenient to introduce the following notation. The standard volume form over $\mathbb{R}^{4}$ is denoted by $d^{4} x=d x^{0} d x^{1} d x^{2} d x^{3}$; the product of forms is understood as wedge product. The symbol $d^{3} x$ means the 3 -form $d^{3} x=$ $d x^{1} d x^{2} d x^{3}$ on $\mathbb{R}^{4}$. Contraction of a form $\omega$ with a vector $v$ is denoted by $i_{v}(\omega)$. The notation $i_{v}(\omega)$ is also used for the spinor matrix valued vector $\gamma=\left(\gamma^{0}, \gamma^{1}, \gamma^{2}, \gamma^{3}\right)=\gamma^{\mu} e_{\mu}$ :

$$
i_{\gamma}\left(d^{4} x\right)=\gamma^{\mu} i_{e_{\mu}}\left(d^{4} x\right) .
$$

Furthermore, for a 4 -spinor $\psi \in \mathbb{C}^{4}$ (viewed as column vector), $\bar{\psi}$ stands for the row vector $\psi^{*} \gamma^{0}$, where ${ }^{*}$ denotes hermitian conjugation.

Definition 2.4 (Spaces of Initial Data in Space-Time Representation). For any Cauchy surface $\Sigma$ we define the vector space

$$
\mathcal{C}_{\Sigma}:=C_{c}^{\infty}\left(\Sigma, \mathbb{C}^{4}\right) .
$$

For a given Cauchy surface $\Sigma$, let $\mathcal{H}_{\Sigma}=L^{2}\left(\Sigma, \mathbb{C}^{4}\right)$ denote the vector space of all 4 -spinor valued measurable functions $\phi: \Sigma \rightarrow \mathbb{C}^{4}$ (modulo changes on null sets) having a finite norm $\|\phi\|=\sqrt{\langle\phi, \phi\rangle}<\infty$ w.r.t. the scalar product

$$
\langle\phi, \psi\rangle=\int_{\Sigma} \overline{\phi(x)} i_{\gamma}\left(d^{4} x\right) \psi(x) .
$$

To see that the pairing in (10) is a scalar product on $\mathcal{C}_{\Sigma} \subset \mathcal{H}_{\Sigma}$, note that for all futuredirected time-like vectors $n$, the $4 \times 4$ matrix

$$
\gamma^{0} \not h=\left(\gamma^{0} \not\right)^{*}
$$

is positive definite. Furthermore, condition (c) of Definition 2.2 ensures for all Cauchy surfaces $\Sigma$

$$
\sup _{x \in \Sigma}\left\|\left(\gamma^{0} \not h(x)\right)^{-1}\right\|<\infty,
$$

where the symbol $\|\cdot\|$ denotes the appropriate matrix norm. In the special case $\Sigma^{0}=\{x \in$ $\left.\mathbb{R}^{4} \mid x^{0}=0\right\}$ the scalar product on $\mathcal{C}_{\Sigma^{0}}$ reduces to the standard one:

$$
\langle\phi, \psi\rangle=\int_{\Sigma^{0}} \phi(x)^{*} \psi(x) d^{3} x .
$$

Note that $\mathcal{C}_{\Sigma}$ is dense in $\mathcal{H}_{\Sigma}$ w.r.t. the scalar product (10). 
Remark 2.5. For $x \in \Sigma$, the restriction of the spinor matrix valued 3-form $i_{\gamma}\left(d^{4} x\right)$ to the tangential space $T_{x} \Sigma$ is given by

$$
i_{\gamma}\left(d^{4} x\right)=\not h(x) i_{n}\left(d^{4} x\right)=\left(\gamma^{0}+\sum_{\mu=1}^{3} \gamma^{\mu} \frac{\partial t_{\Sigma}(\mathbf{x})}{\partial x^{\mu}}\right) d^{3} x \text { on }\left(T_{x} \Sigma\right)^{3},
$$

where $n$ denotes the future-directed unit normal vector field to $\Sigma$ in the Minkowski sense. In physics one often uses the notation $d \sigma(x)=i_{n}\left(d^{4} x\right)$.

Finally, the space of solutions $\mathcal{C}_{A}$ can be extended to a Hilbert space:

Definition 2.6 (Hilbert Space of Solutions in Space-Time Representation). We endow $\mathcal{C}_{A}$ with the scalar product

$$
\langle\phi, \psi\rangle=\int_{\Sigma} \overline{\phi(x)} i_{\gamma}\left(d^{4} x\right) \psi(x)
$$

where $\Sigma$ denotes any Cauchy surface and define

$$
\mathcal{H}_{A}:=\operatorname{completion}\left(\mathcal{C}_{A}\right)
$$

which denotes the (abstract) completion of $\mathcal{C}_{A}$ w.r.t. the norm $\|\psi\|=\sqrt{\langle\psi, \psi\rangle}$. In case the external potential $A$ is zero we will use the notation $\mathcal{C}_{0}=\left.\mathcal{C}_{A}\right|_{A=0}$.

Note that the scalar product (15) is well-defined for $\phi, \psi \in \mathcal{C}_{A}$ because the support of the form $\overline{\phi(x)} i_{\gamma}\left(d^{4} x\right) \psi(x)$ intersects $\Sigma$ in a compact set, and because the integral does not depend on the choice of the Cauchy surface $\Sigma$. This follows from Stokes' theorem as the 3 -form $\overline{\phi(x)} i_{\gamma}\left(d^{4} x\right) \psi(x)$ is closed:

$$
\begin{aligned}
& d\left[\overline{\phi(x)} i_{\gamma}\left(d^{4} x\right) \psi(x)\right]=\partial_{\mu}\left(\overline{\phi(x)} \gamma^{\mu} \psi(x)\right) d^{4} x \\
& =\left(\partial_{\mu} \overline{\phi(x)}\right) \gamma^{\mu} \psi(x) d^{4} x+\overline{\phi(x)} \gamma^{\mu} \partial_{\mu} \psi(x) d^{4} x \\
& =\overline{\not \phi(x)} \psi(x) d^{4} x+\overline{\phi(x)} \not \psi(x) d^{4} x \\
& =\overline{(m+\not) \phi(x)} \psi(x) d^{4} x-i \overline{\phi(x)}(m+\not) \psi(x) d^{4} x=0 .
\end{aligned}
$$

\subsection{Relevant Spaces in Energy-Momentum Representation}

In the same way the momentum representation, i.e., the standard Fourier transform, provides

a fundamental tool for the study of the non-relativistic Schrödinger equation on equal-time hyperplanes, the energy-momentum representation of solutions in $\mathcal{H}_{0}=\mathcal{H}_{A=0}$ and the corresponding generalized Fourier transform will facilitate the study of the Dirac equation on Cauchy surfaces. For this purpose we introduce the mass shell

$$
\mathcal{M}=\left\{p \in \mathbb{R}^{4} \mid p_{\mu} p^{\mu}=m^{2}\right\} .
$$

The mass shell $\mathcal{M}$ has two connected components which are denoted by

$$
\mathcal{M}_{+}=\left\{p \in \mathcal{M} \mid p^{0}>0\right\}, \quad \mathcal{M}_{-}=\left\{p \in \mathcal{M} \mid p^{0}<0\right\}
$$


We endow $\mathcal{M}$ with the orientation that makes the projection $\mathcal{M} \rightarrow \mathbb{R}^{3},\left(p^{0}, \mathbf{p}\right) \mapsto \mathbf{p}$ positively oriented. Restricted to $\mathcal{M}_{ \pm}$, this projection has the inverses

$$
\mathbb{R}^{3} \ni \mathbf{p} \mapsto p_{ \pm}(\mathbf{p})=( \pm E(\mathbf{p}), \mathbf{p}) \in \mathcal{M}_{ \pm}, \quad \text { where } \quad E(\mathbf{p}):=\sqrt{\mathbf{p}^{2}+m^{2}}
$$

The free Dirac equation in momentum representation reads $\not p \psi=m \psi$ for $p \in \mathcal{M}$. The corresponding solution space is given by

$$
\mathcal{D}_{p}=\left\{\psi \in \mathbb{C}^{4} \mid \not p \psi=m \psi\right\}
$$

We therefore introduce the complex vector bundle of rank 2 over $\mathcal{M}$

$$
\mathcal{D}:=\left\{(p, \psi) \mid p \in \mathcal{M}, \psi \in \mathcal{D}_{p}\right\}
$$

which we call the Dirac bundle. For $p \in \mathcal{M}$, the orthogonal projection from $\mathbb{C}^{4}$ onto $\mathcal{D}_{p}$ w.r.t. the standard scalar product is given by the matrix

$$
P(p)=\frac{\not p+m}{2 p^{0}} \gamma^{0}
$$

For $\mathbf{p} \in \mathbb{R}^{3}$, the vector spaces $\mathcal{D}_{p_{+}(\mathbf{p})}$ and $\mathcal{D}_{p_{-}(\mathbf{p})}$ are orthogonal complements to each other so that

$$
P_{+}(\mathbf{p})+P_{-}(\mathbf{p})=1 \in \mathbb{C}^{4 \times 4},
$$

where we used the short-hand notation

$$
P_{ \pm}(\mathbf{p})=P\left(p_{ \pm}(\mathbf{p})\right)
$$

With the application of Paley-Wiener arguments in mind to study support properties of functions, we also introduce a complexified version $\mathcal{M}_{\mathbb{C}}$. We define

$$
\mathcal{M}_{\mathbb{C}}=\left\{p \in \mathbb{C}^{4} \mid p_{\mu} p^{\mu}=m^{2}\right\}
$$

Note that $\mathcal{M}_{\mathbb{C}}$ is a connected submanifold of $\mathbb{C}^{4}$ of complex dimension 3 . We use the following notations. The standard volume form over $\mathbb{R}^{3}$ is denoted by $d^{3} \mathbf{p}=d p^{1} d p^{2} d p^{3}$ and one has

$$
i_{p}\left(d^{4} p\right)=p^{0} d p^{1} d p^{2} d p^{3}-p^{1} d p^{0} d p^{2} d p^{3}+p^{2} d p^{0} d p^{1} d p^{3}-p^{3} d p^{0} d p^{1} d p^{2} .
$$

For $p \in \mathcal{M}$, the restriction of this form to the tangential space $T_{p} \mathcal{M}$ is the Lorentz invariant volume form on the mass shell

$$
i_{p}\left(d^{4} p\right)=\frac{m^{2}}{p^{0}} d p^{1} d p^{2} d p^{3}=\frac{m^{2}}{p^{0}} d^{3} p \text { on }\left(T_{p} \mathcal{M}\right)^{3} .
$$

The euclidean norm of $p \in \mathbb{C}^{d}$ for $d \in \mathbb{N}$ is denoted by $|p|$. We introduce the following spaces:

Definition 2.7 (Solutions in Energy-Momentum Representation). Let $\mathcal{H}_{\mathcal{M}}=L^{2}(\mathcal{M}, \mathcal{D})$ denote the space of all square integrable sections $\psi$ in the Dirac bundle. This means that 
$\mathcal{H}_{\mathcal{M}}$ consists of all measurable functions $\psi: \mathcal{M} \rightarrow \mathbb{C}^{4}$ (modulo changes on null sets) that fulfill almost everywhere

$$
\not p \psi(p)=m \psi(p)
$$

for $p \in \mathcal{M}$ and $\|\psi\|=\sqrt{\langle\psi, \psi\rangle}<\infty$, with the scalar product

$$
\langle\phi, \psi\rangle=\int_{\mathcal{M}} \overline{\phi(p)} \psi(p) \frac{i_{p}\left(d^{4} p\right)}{m} .
$$

Let $\mathcal{C}_{\mathcal{M}} \subseteq \mathcal{H}_{\mathcal{M}}$ denote the subspace of all functions $\psi \in \mathcal{H}_{\mathcal{M}}$ that have a holomorphic continuation $\Psi: \mathcal{M}_{\mathbb{C}} \rightarrow \mathbb{C}^{4}$ fulfilling the bound

$$
\exists \alpha>0 \forall n \in \mathbb{N}:\|\psi\|_{\mathcal{M}, \alpha, n}<\infty
$$

with

$$
\|\psi\|_{\mathcal{M}, \alpha, n}:=\sup _{p \in \mathcal{M}_{\mathbb{C}}}|p|^{n-1} e^{-\alpha|\operatorname{Im} \mathbf{p}|}|\Psi(p)| .
$$

It is shown in Corollary 3.3 below that $\mathcal{C}_{\mathcal{M}}$ is dense in $\mathcal{H}_{\mathcal{M}}$. It turns out that the norms (32) involving only the 3 -vector part $\operatorname{Im} \mathbf{p}$, and not the 4 -vector $\operatorname{Im} p$, in the exponent are more convenient to use, and regarding Lorentz invariance the particular choice makes no difference as we shall see in Section 2.3 below.

Note that the holomorphic continuation $\Psi$ is uniquely determined by $\psi \in \mathcal{C}_{\mathcal{M}}$. Even more, it is already determined by its restriction to any non-empty relatively to $\mathcal{M}$ open subset $U \subseteq \mathcal{M}$. In particular, the restriction of $\psi$ to $\mathcal{M}_{+}$already determines its values on $\mathcal{M}_{-}$and vice versa. Furthermore, the condition $\psi(p) \in \mathcal{D}_{p}$ for all $p \in \mathcal{M}$ extends analytically to

$$
\not p \Psi(p)=m \Psi(p) \quad \text { for all } p \in \mathcal{M}_{\mathbb{C}}
$$

Note further that the inner product (30) is positive definite. This can be seen as follows. For $p \in \mathcal{M}$, the facts $\phi(p) \in \mathcal{D}_{p}$ and $\gamma^{0} \not p=\not p^{*} \gamma^{0}$ imply $\overline{\phi(p)} \not p=m \overline{\phi(p)}$ and thus

$$
m \overline{\phi(p)} \gamma^{\mu} \psi(p)=\overline{\phi(p)} \gamma^{\mu} \not p \psi(p)=\overline{\phi(p)}\left(2 p^{\mu}-\not p \gamma^{\mu}\right) \psi(p)=\overline{\phi(p)}\left(2 p^{\mu}-m \gamma^{\mu}\right) \psi(p) .
$$

We conclude

$$
m \overline{\phi(p)} \gamma^{\mu} \psi(p)=p^{\mu} \overline{\phi(p)} \psi(p)
$$

Using (28), we get

$$
\langle\phi, \psi\rangle=\int_{\mathcal{M}} \overline{\phi(p)} \frac{p^{0}}{p^{0}} \psi(p) \frac{i_{p}\left(d^{4} p\right)}{m}=\int_{\mathcal{M}} \overline{\phi(p)} \gamma^{0} \psi(p) \frac{i_{p}\left(d^{4} p\right)}{p^{0}}=m^{2} \int_{\mathcal{M}} \phi(p)^{*} \psi(p) \frac{d^{3} p}{\left(p^{0}\right)^{2}},
$$

which is positive for $\phi=\psi$ unless $\psi=0$ almost everywhere.

As it turns out the causal structure of solutions to the Dirac equation can be seen to emerge from the following geometric property of $\mathcal{M}_{\mathbb{C}}$, proven in Lemma A.1 in the appendix:

$$
\left|\operatorname{Im} p^{0}\right| \leqslant|\operatorname{Im} \mathbf{p}| \quad \text { for all }\left(p^{0}, \mathbf{p}\right) \in \mathcal{M}_{\mathbb{C}}
$$


Finally, it will be convenient to introduce a Hilbert space $\mathcal{H}_{3}$ for the 3 -momentum representation as it allows to fall back on many classical results about the standard Fourier transform. In view of the Paley-Wiener theorem we define also a subspace $\mathcal{C}_{3} \subset \mathcal{H}_{3}$ consisting of certain real-analytic functions.

Definition 2.8. We endow $\mathcal{H}_{3}:=L^{2}\left(\mathbb{R}^{3}, \mathbb{C}^{4}\right)$ with the standard scalar product

$$
\langle\phi, \psi\rangle=\int_{\mathbb{R}^{3}} \phi^{*}(\mathbf{p}) \psi(\mathbf{p}) d^{3} \mathbf{p}
$$

and the corresponding norm $\|\phi\|=\sqrt{\langle\phi, \phi\rangle}$. Let $\mathcal{C}_{3}$ be the subspace of $\mathcal{H}_{3}$ consisting of all functions $\phi: \mathbb{R}^{3} \rightarrow \mathbb{C}^{4}$ that have a holomorphic continuation $\Phi: \mathbb{C}^{3} \rightarrow \mathbb{C}^{4}$ that fulfills the bound

$$
\exists \alpha>0 \forall n \in \mathbb{N}_{0}:\|\phi\|_{3, \alpha, n}<\infty,
$$

where we set (using the notation $a \vee b=\max \{a, b\}$ )

$$
\|\phi\|_{3, \alpha, n}:=\sup _{\mathbf{p} \in \mathbb{C}^{3}}(m \vee|\mathbf{p}|)^{n} e^{-\alpha|\operatorname{Im} \mathbf{p}|}|\Phi(\mathbf{p})| .
$$

This is well-defined as $\Phi$ is uniquely determined by $\phi$.

Lemma 2.9. $\mathcal{C}_{3}$ is dense in $\mathcal{H}_{3}$.

Proof. By the classical Paley-Wiener Theorem [RS81, Theorem IX.11] the Fourier transform $L^{2}\left(\mathbb{R}^{3}, \mathbb{C}^{4}\right) \rightarrow \mathcal{H}_{3}$ maps $\mathcal{C}_{c}^{\infty}\left(\mathbb{R}^{3}, \mathbb{C}^{4}\right)$ bijectively onto $\mathcal{C}_{3}$. Because the Fourier transform is unitary and $\mathcal{C}_{c}^{\infty}\left(\mathbb{R}^{3}, \mathbb{C}^{4}\right)$ is dense in $L^{2}\left(\mathbb{R}^{3}, \mathbb{C}^{4}\right)$ the claim follows.

\subsection{Action of the Poincaré Group}

In our later analysis, Poincaré transformations will prove to be very helpful in computations. First, we introduce the Lorentz transformations on 4 -spinors $\psi \in \mathbb{C}^{4}$ and on space-time points $x \in \mathbb{R}^{4}$. They are specified by a pair $(S, \Lambda)$ with a spinor matrix $S \in \mathbb{C}^{4 \times 4}$ and a matrix $\Lambda=\left(\Lambda^{\mu}{ }_{\nu}\right)_{\mu, \nu=0,1,2,3} \in \mathbb{R}^{4 \times 4}$ that fulfill

$$
\Lambda_{\sigma}^{\mu} g_{\mu \nu} \Lambda_{\tau}^{\nu}=g_{\sigma \tau}, \quad S^{*} \gamma^{0} S=\gamma^{0}
$$

and are related by

$$
\Lambda_{\nu}^{\mu} \gamma^{\nu}=S^{-1} \gamma^{\mu} S
$$

Space-time points $x \in \mathbb{R}^{4}$ and Dirac spinor fields $\psi: \mathbb{R}^{4} \rightarrow \mathbb{C}^{4}$ are transformed by

$$
\begin{aligned}
x^{\prime \mu} & =\Lambda^{\mu}{ }_{\nu} x^{\nu}, \\
\psi^{\prime}\left(x^{\prime}\right) & =S \psi(x) .
\end{aligned}
$$

Let

$$
T=\frac{1}{\sqrt{2}}\left(\begin{array}{cc}
1 & 1 \\
1 & -1
\end{array}\right)=T^{-1} \in \mathbb{C}^{4 \times 4}
$$


be the unitary transformation matrix from the standard representation of Dirac spinors to the Weyl spinor representation; see [Tha92, Appendix 1.A]. Transformations associated to the proper, orthochronous Lorentz group $\mathrm{SO}^{\uparrow}(1,3)$ are parametrized by

$$
\mathrm{SL}(2, \mathbb{C}) \ni M \mapsto(S(M), \Lambda(M)) \in \mathrm{GL}(4, \mathbb{C}) \times \mathrm{SO}^{\uparrow}(1,3),
$$

where

$$
S(M)=T^{-1}\left(\begin{array}{cc}
M & 0 \\
0 & \left(M^{*}\right)^{-1}
\end{array}\right) T \in \mathbb{C}^{4 \times 4}
$$

and $\Lambda(M) \in \mathbb{R}^{4 \times 4}$ is the unique matrix such that equation (42) holds for $S=S(M)$ and $\Lambda=\Lambda(M)$. Note that the map (46) is a group homomorphism.

Let $m_{\Lambda}: \mathbb{R}^{4} \rightarrow \mathbb{R}^{4}, x \mapsto x^{\prime}=\Lambda x$, denote multiplication with $\Lambda$. Using equation (42), we observe the following Lorentz covariance relations for pull-back w.r.t. this map:

$$
\begin{aligned}
S^{-1}\left[m_{\Lambda}^{*} i_{\gamma}\left(d^{4} x^{\prime}\right)\right] S & =S^{-1} \gamma^{\mu} S m_{\Lambda}^{*} i_{e_{\mu}}\left(d^{4} x^{\prime}\right)=\Lambda_{\nu}^{\mu} \gamma^{\nu} i_{\Lambda^{-1} e_{\mu}}\left(d^{4} x\right)=\gamma^{\nu} i_{e_{\nu}}\left(d^{4} x\right)=i_{\gamma}\left(d^{4} x\right), \\
m_{\Lambda}^{*} i_{p^{\prime}}\left(d^{4} p^{\prime}\right) & =i_{p}\left(d^{4} p\right) .
\end{aligned}
$$

Poincaré transformations act in a natural way on the spaces defined in this section:

Definition 2.10 (Translations and Lorentz Transformations). Let $\Sigma$ be a Cauchy surface and $y \in \mathbb{R}^{4}$. We define the translation maps:

$$
\begin{aligned}
& T_{\Sigma}^{-y}: \mathcal{C}_{\Sigma} \rightarrow \mathcal{C}_{\Sigma-y}, \quad T_{\Sigma}^{-y} \psi(x)=\psi(x+y) \quad \text { for } x \in \Sigma-y \text {; } \\
& T_{A}^{-y}: \mathcal{C}_{A} \rightarrow \mathcal{C}_{A(\cdot+y)}, \quad T_{A}^{-y} \psi(x)=\psi(x+y) \quad \text { for } x \in \mathbb{R}^{4} ; \\
& T_{\mathcal{M}}^{-y}: \mathcal{C}_{\mathcal{M}} \rightarrow \mathcal{C}_{\mathcal{M}}, \quad T_{\mathcal{M}}^{-y} \phi(p)=e^{-i p y} \phi(p) \quad \text { for } p \in \mathcal{M} \text {. }
\end{aligned}
$$

Furthermore, for any $M \in \mathrm{SL}(2, \mathbb{C})$ associated with a proper, orthochronous Lorentz transformation $\Lambda=\Lambda(M)$ and a spinor transformation $S=S(M)$ as in (46) we define the maps

$$
\begin{aligned}
& L_{\Sigma}^{(S, \Lambda)}: \mathcal{C}_{\Sigma} \rightarrow \mathcal{C}_{\Lambda \Sigma}, \quad L_{\Sigma}^{(S, \Lambda)} \psi(x)=S \psi\left(\Lambda^{-1} x\right) \quad \text { for } x \in \Lambda \Sigma ; \\
& L_{A}^{(S, \Lambda)}: \mathcal{C}_{A} \rightarrow \mathcal{C}_{\Lambda A\left(\Lambda^{-1} \cdot\right)}, \quad L_{A}^{(S, \Lambda)} \psi(x)=S \psi\left(\Lambda^{-1} x\right) \quad \text { for } x \in \mathbb{R}^{4} \\
& L_{\mathcal{M}}^{(S, \Lambda)}: \mathcal{C}_{\mathcal{M}} \rightarrow \mathcal{C}_{\mathcal{M}}, \quad L_{\mathcal{M}}^{(S, \Lambda)} \phi(p)=S \phi\left(\Lambda^{-1} p\right) \quad \text { for } p \in \mathcal{M}
\end{aligned}
$$

Lemma 2.11. The six maps specified in Definition 2.10 are well-defined. More precisely, they take their values in the spaces specified in formulas (50)-(55). They extend to unitary maps, also denoted by $T_{\Sigma}^{-y}: \mathcal{H}_{\Sigma} \rightarrow \mathcal{H}_{\Sigma-y}, T_{A}^{-y}: \mathcal{H}_{A} \rightarrow \mathcal{H}_{A(\cdot+y)}, T_{\mathcal{M}}^{-y}: \mathcal{H}_{\mathcal{M}} \rightarrow \mathcal{H}_{\mathcal{M}}$, $L_{\Sigma}^{(S, \Lambda)}: \mathcal{H}_{\Sigma} \rightarrow \mathcal{H}_{\Lambda \Sigma}, L_{A}^{(S, \Lambda)}: \mathcal{H}_{A} \rightarrow \mathcal{H}_{\Lambda A\left(\Lambda^{-1} \cdot\right)}$, and $L_{\mathcal{M}}^{(S, \Lambda)}: \mathcal{H}_{\mathcal{M}} \rightarrow \mathcal{H}_{\mathcal{M}}$, respectively

The proof is given in Appendix A.

\subsection{Change of Gauge}

Another physically relevant transformation is the change of gauge in the electrodynamic potential $A$. The transformation of the potential is defined as

$$
A_{\mu}^{\prime}(x) \mapsto A_{\mu}(x)+\partial_{\mu} \lambda(x)
$$

for any scalar field $\lambda \in \mathcal{C}_{c}^{\infty}\left(\mathbb{R}^{4}, \mathbb{R}\right)$. 
Definition 2.12 (Gauge Transformation). Let $\lambda \in \mathcal{C}_{c}^{\infty}\left(\mathbb{R}^{4}, \mathbb{R}\right)$. We define

$$
\Gamma_{\lambda}: \mathcal{C}_{A} \rightarrow \mathcal{C}_{A+\partial \lambda}, \quad \Gamma_{\lambda} \psi(x)=e^{-i \lambda(x)} \psi(x) \quad \text { for } x \in \mathbb{R}^{4},
$$

Lemma 2.13. The maps specified in Definition 2.12 are well-defined. More precisely, they take their values in the spaces specified in formula (57). They extend to unitary maps, also denoted by $\Gamma_{\lambda}: \mathcal{H}_{A} \rightarrow \mathcal{H}_{A+\partial \lambda}$.

The proof is given in Appendix A.

\subsection{Generalized Fourier Transforms}

Next we introduce the mentioned generalized Fourier transforms. Their properties are collected in the following main theorem. An immediate byproduct is an evolution operator for the free Dirac equation, i.e., equation (1) for $A=0$. In the following we write

$$
p x=p_{\mu} x^{\mu}=p^{0} x^{0}-p^{1} x^{1}-p^{2} x^{2}-p^{3} x^{3}=p^{0} x^{0}-\mathbf{p} \cdot \mathbf{x}, \quad x, p \in \mathbb{C}^{4} .
$$

The two different meanings of $p^{2}$ as second component of $p \in \mathbb{C}^{4}$ and $p^{2}=p_{\mu} p^{\mu}$ will be unambiguous given the context.

Theorem 2.14 (Generalized Fourier Transforms and Free Dirac Evolution).

(a) For all $I, J, K$ being placeholders for the symbols $3, \mathcal{M}, 0$ or any Cauchy surface $\Sigma$ there are unique unitary maps $\mathcal{F}_{I J}: \mathcal{H}_{J} \rightarrow \mathcal{H}_{I}$ with the following properties:

(i) $\mathcal{F}_{I I}=\operatorname{id}_{\mathcal{H}_{I}}$.

(ii) $\mathcal{F}_{I J} \mathcal{F}_{J K}=\mathcal{F}_{I K}$.

(iii) $\mathcal{F}_{I J}$ maps $\mathcal{C}_{J}$ bijectively onto $\mathcal{C}_{I}$.

(iv) The maps $\mathcal{F}_{3 \mathcal{M}}, \mathcal{F}_{\mathcal{M} 3}, \mathcal{F}_{\mathcal{M} \Sigma}, \mathcal{F}_{0 \mathcal{M}}$, and $\mathcal{F}_{\Sigma 0}$ are characterized as follows:

$$
\begin{aligned}
\left(\mathcal{F}_{3 \mathcal{M}} \psi\right)(\mathbf{p}) & =m \frac{\psi\left(p_{+}(\mathbf{p})\right)-\psi\left(p_{-}(\mathbf{p})\right)}{E(\mathbf{p})} & & \text { for } \psi \in \mathcal{H}_{\mathcal{M}}, \mathbf{p} \in \mathbb{R}^{3} ; \\
\left(\mathcal{F}_{\mathcal{M} 3} \psi\right)(p) & =\frac{\not p+m}{2 m} \gamma^{0} \psi(\mathbf{p}) & & \text { for } \psi \in \mathcal{H}_{3}, p=\left(p^{0}, \mathbf{p}\right) \in \mathcal{M} ; \\
\left(\mathcal{F}_{\mathcal{M} \Sigma} \psi\right)(p) & =\frac{\not p+m}{2 m}(2 \pi)^{-3 / 2} \int_{\Sigma} e^{i p x} i_{\gamma}\left(d^{4} x\right) \psi(x) & & \text { for } \psi \in \mathcal{C}_{\Sigma}, p \in \mathcal{M} ; \\
\left(\mathcal{F}_{0 \mathcal{M}} \psi\right)(x) & =\frac{(2 \pi)^{-3 / 2}}{m} \int_{\mathcal{M}} e^{-i p x} i_{p}\left(d^{4} p\right) \psi(p) & & \text { for } \psi \in \mathcal{C}_{\mathcal{M}}, x \in \mathbb{R}^{4} ; \\
\left(\mathcal{F}_{\Sigma 0} \psi\right)(x) & =\psi(x) & & \text { for } \psi \in \mathcal{C}_{0}, x \in \Sigma .
\end{aligned}
$$

(b) For $\psi \in \mathcal{C}_{\Sigma}$, the function $\mathcal{F}_{0 \Sigma} \psi$ is supported in $\operatorname{supp} \psi+$ Causal.

(c) For any symbol I among $3, \mathcal{M}, \Sigma, 0$ the space $\mathcal{C}_{I}$ is dense in $\mathcal{H}_{I}$. 
The free Dirac evolution between Cauchy surfaces $\Sigma$ and $\Sigma^{\prime}$ is given by the unitary map $\mathcal{F}_{\Sigma^{\prime} \Sigma}$. In physicists' notation the formal integral kernel of $\mathcal{F}_{0 \Sigma}$ is usually called the propagator of the free Dirac equation. The maps $\mathcal{F}_{\mathcal{M} \Sigma}$ and $\mathcal{F}_{0 \mathcal{M}}$ commute with Poincaré transformations in the following sense:

Theorem 2.15 (Compatibility with Poincaré Transformations). For any translation vector $y \in \mathbb{R}^{4}$ and any Lorentz transformation associated with $\Lambda=\Lambda(M), S=S(M)$ with $M \in$ $\mathrm{SL}(2, \mathbb{C})$, cf. Definition 2.10, the following compatibility relations hold true.

$$
\begin{aligned}
T_{\mathcal{M}}^{-y} \mathcal{F}_{\mathcal{M} \Sigma} & =\mathcal{F}_{\mathcal{M}, \Sigma-y} T_{\Sigma}^{-y} \\
T_{0}^{-y} \mathcal{F}_{0 \mathcal{M}} & =\mathcal{F}_{0 \mathcal{M}} T_{\mathcal{M}}^{-y} \\
L_{\mathcal{M}}^{(S, \Lambda)} \mathcal{F}_{\mathcal{M} \Sigma} & =\mathcal{F}_{\mathcal{M}, \Lambda \Sigma} L_{\Sigma}^{(S, \Lambda)} \\
L_{0}^{(S, \Lambda)} \mathcal{F}_{0 \mathcal{M}} & =\mathcal{F}_{0 \mathcal{M}} L_{\mathcal{M}}^{(S, \Lambda)}
\end{aligned}
$$

The proof of Theorem 2.14 is given in Section 3.1.3, and the proof of Theorem 2.15 is given in Appendix A. While the latter is straight-forward the former needs several technical lemmas, some of which are phrased in the following subsection; the remaining technical lemmas and proofs are given in Section 3.1. Note that beside the regularity information contained in the spaces $\mathcal{C}_{\Sigma}$ and $\mathcal{C}_{0}$ Theorem 2.14 (b) makes precise the causal structure of the support properties of solutions of the free Dirac equation. These support properties are controlled by Paley-Wiener techniques. The standard Paley-Wiener theorem treats the case in which both position and momentum spaces are flat. To apply this theorem to curved Cauchy surfaces we employ a family of projections from a particular Cauchy surface to a flat position space; see proof of Lemma 3.4. On the momentum space side, the mass-shell $\mathcal{M}$ is analytically continued to a complex 3-dimensional manifold $\mathcal{M}_{\mathbb{C}}$ which is also projected onto a complexified 3-momentum space $\mathbb{C}^{3}$. The Paley-Wiener theorem is then applied not to the holomorphic functions on $\mathcal{M}_{\mathbb{C}}$ directly but to their appropriate projections. Tools from complex analysis help to control the projected functions quantitatively, in particular close to the ramification set of the projection; see proof of Lemma 3.1. The following section provides an overview of the most important bounds, and in Section 2.5.2 we introduce appropriate Sobolev norms that are also helpful to control regularity of solutions of the Dirac equation subject to an external potential $A$; see Section 3.2.

\subsubsection{Paley-Wiener Bounds}

Let $K \subset \mathbb{R}^{4}$ be a compact set and $0 \leqslant V<1$. We define $\mathcal{S}(K, V)$ to be the set of all Cauchy surfaces $\Sigma$ with $\Sigma \cap K \neq \varnothing$ and $\sup _{\mathbf{x} \in \mathbb{R}^{3}}\left|\nabla t_{\Sigma}(\mathbf{x})\right| \leqslant V$; cf. (7). For $\Sigma \in \mathcal{S}(K, V)$ let $\mathcal{C}_{\Sigma}(K)$ denote the set of all wave functions $\psi \in \mathcal{C}_{\Sigma}$ supported in $K \cap \Sigma$. For such $\psi$ and $n \in \mathbb{N}_{0}$ we define

$$
\|\psi\|_{\Sigma, K, n}=\sup _{\mathbf{x} \in \mathbb{R}^{3}} \sum_{|\beta| \leqslant n}\left|D^{\beta} \psi\left(t_{\Sigma}(\mathbf{x}), \mathbf{x}\right)\right|,
$$

where the differential operator $D^{\beta}$ for a multi-index $\beta \in \mathbb{N}_{0}^{3}$ acts on $\mathbf{x} \in \mathbb{R}^{3}$. 
Theorem 2.16 (Paley-Wiener Bounds for Cauchy Surfaces). For any $K, V$ as above, any $\Sigma \in \mathcal{S}(K, V)$, any $\psi \in \mathcal{C}_{\Sigma}(K)$, any positive number $\alpha$ such that $\alpha>\sqrt{2} \sup _{x \in K}|x|$, and any $n \in \mathbb{N}$ one has

$$
\left\|\mathcal{F}_{\mathcal{M} \Sigma} \psi\right\|_{\mathcal{M}, \alpha, n} \leqslant C_{1}\|\psi\|_{\Sigma, K, n}
$$

with some positive constant $C_{1}=C_{1}(K, V, n, \alpha, m)$.

This theorem is proven in the proof of Lemma 3.4. Furthermore, we give the following bounds which are useful in switching between the spaces $\mathcal{C}_{\mathcal{M}}$ and $\mathcal{C}_{3}$.

Theorem 2.17 (Bounds on $\mathcal{C}_{\mathcal{M}}$ and $\mathcal{C}_{3}$ ). For any $\alpha>0, \epsilon>0$, and $n \in \mathbb{N}$ the following bounds hold

$$
\begin{aligned}
\left\|\mathcal{F}_{3 \mathcal{M}} \psi\right\|_{3, \alpha, n-1} & \leqslant C_{3}\|\psi\|_{\mathcal{M}, \alpha, n} & & \text { for } \psi \in \mathcal{C}_{\mathcal{M}}, \\
\left\|\mathcal{F}_{3 \mathcal{M}} \psi\right\|_{3, \alpha, n} & \leqslant C_{4}\|\psi\|_{\mathcal{M}, \alpha+\epsilon, n} & & \text { for } \psi \in \mathcal{C}_{\mathcal{M}}, \\
\left\|\mathcal{F}_{\mathcal{M} 3} \phi\right\|_{\mathcal{M}, \alpha, n} & \leqslant C_{5}\|\phi\|_{3, \alpha, n} & & \text { for } \phi \in \mathcal{C}_{3},
\end{aligned}
$$

with positive constants $C_{3}=C_{3}(n, \alpha, m), C_{4}=C_{4}(n, \alpha, \epsilon, m)$, and $C_{5}=C_{5}(n, m)$.

This theorem is proven in the proof of Lemma 3.1.

\subsubsection{Sobolev Norms}

On the one hand, the norms $\|\cdot\|_{\mathcal{M}, \alpha, n}$, introduced in (32) are well adapted to Paley-Wiener arguments and are therefore useful for the analysis of support properties. On the other hand, Sobolev norms turn out to be more convenient for the analysis of regularity. Now we introduce a version of Sobolev norms well suited for the analysis of the Dirac equation.

Definition 2.18. For $n \in \mathbb{N}_{0}$, let $\mathcal{H}_{\mathcal{M}, n}$ denote the vector space of all $\psi \in \mathcal{H}_{\mathcal{M}}$ such that $p^{\beta} \psi \in \mathcal{H}_{\mathcal{M}}$ for any multi-index $\beta \in \mathbb{N}_{0}^{4}$ with $|\beta| \leqslant n$. Here $p^{\beta}:=p_{0}^{\beta_{0}} p_{1}^{\beta_{1}} p_{2}^{\beta_{2}} p_{3}^{\beta_{3}}$, where $p_{j}$ stands for the multiplication operator with $p_{j}, p \in \mathcal{M}$. We endow $\mathcal{H}_{\mathcal{M}, n}$ with the norm

$$
\|\psi\|_{\mathcal{M}, n}^{2}:=\sum_{\substack{\beta \in \mathbb{N}_{0}^{4} \\|\beta| \leqslant n}}\left\|p^{\beta} \psi\right\|^{2}=\sum_{\substack{\beta \in \mathbb{N}_{0}^{4} \\|\beta| \leqslant n}} \int_{\mathcal{M}}\left|p^{\beta}\right|^{2} \overline{\psi(p)} \psi(p) \frac{i_{p}\left(d^{4} p\right)}{m} .
$$

Given a Cauchy surface $\Sigma$, for a placeholder I standing for 0 or $\Sigma$, we define the normed space

$$
\mathcal{H}_{I, n}:=\mathcal{F}_{I \mathcal{M}}\left[\mathcal{H}_{\mathcal{M}, n}\right], \quad\|\psi\|_{I, n}:=\left\|\mathcal{F}_{\mathcal{M} I} \psi\right\|_{\mathcal{M}, n}
$$

and for any $j=0,1,2,3$ the bounded operator

$$
\partial_{j}: \mathcal{H}_{I, n+1} \rightarrow \mathcal{H}_{I, n}, \quad \psi \mapsto-i \mathcal{F}_{I \mathcal{M}} p_{j} \mathcal{F}_{\mathcal{M I}} \psi
$$


We remark that for any placeholder $I$, standing for $\mathcal{M}, 0$, or $\Sigma$, the space $\left(\mathcal{H}_{I, n},\|\cdot\|_{I, n}\right)$ is a Hilbert space containing $\mathcal{C}_{I}$ as a dense subspace. Furthermore, the multiplication operator $p^{\beta}:\left(\mathcal{H}_{\mathcal{M}, n},\|\cdot\|_{\mathcal{M}, n}\right) \rightarrow\left(\mathcal{H}_{\mathcal{M}, n-|\beta|},\|\cdot\|_{\mathcal{M}, n-|\beta|}\right)$ is bounded. Note that the restriction of $\partial_{j}:$ $\mathcal{H}_{0, n+1} \rightarrow \mathcal{H}_{0, n}$ to $\mathcal{C}_{0}$ is the differential operator $\partial_{j} \psi(x)=\frac{\partial}{\partial x^{j}} \psi(x)$. For $\psi \in \mathcal{H}_{\mathcal{M}, n+1}, n \geqslant 0$, and $\widehat{\psi}=\mathcal{F}_{\mathcal{M} 0} \psi$ one has

$$
\left\|\partial_{j} \psi\right\|_{0, n}=\left\|p_{j} \widehat{\psi}\right\|_{\mathcal{M}, n}
$$

Thanks to the free Dirac equation, the restriction of $\partial_{j}: \mathcal{H}_{\Sigma, n+1} \rightarrow \mathcal{H}_{\Sigma, n}$ to $\mathcal{C}_{\Sigma}$ is also a differential operator. For $\mathbf{x}=\left(x^{1}, x^{2}, x^{3}\right) \in \mathbb{R}^{3}$ it takes the form

$$
\partial_{j} \psi(x)=-i\left(\sum_{k=1}^{3} \alpha_{j k}^{\Sigma}(x) D_{k}+\beta_{j}^{\Sigma}(x)\right) \psi(x), \quad x=\left(t_{\Sigma}(\mathbf{x}), \mathbf{x}\right),
$$

with some smooth functions $\alpha_{j k}^{\Sigma}, \beta_{j}^{\Sigma}: \Sigma \rightarrow \mathbb{C}^{4 \times 4}$ depending only on the geometry of $\Sigma$ and

$$
D_{k} \psi\left(t_{\Sigma}(\mathbf{x}), \mathbf{x}\right)=\frac{\partial}{\partial x^{k}}\left(\psi\left(t_{\Sigma}(\mathbf{x}), \mathbf{x}\right)\right)=\partial_{k} \psi\left(t_{\Sigma}(\mathbf{x}), \mathbf{x}\right)+\frac{\partial t_{\Sigma}(\mathbf{x})}{\partial x^{k}} \partial_{0} \psi\left(t_{\Sigma}(\mathbf{x}), \mathbf{x}\right)
$$

The following lemma shows that pointwise evaluation for elements of $\mathcal{H}_{0, n}$ makes sense whenever $n \geqslant 2$.

Lemma 2.19 (Pointwise Evaluation). For $n \in \mathbb{N}$ with $n \geqslant 2$ and $x \in \mathbb{R}^{4}$, the evaluation map $\delta_{x}: \mathcal{C}_{0} \rightarrow \mathbb{C}^{4}, \psi \mapsto \psi(x)$, extends to a bounded linear map $\delta_{x}:\left(\mathcal{H}_{0, n},\|\cdot\|_{0, n}\right) \rightarrow \mathbb{C}^{4}$, also denoted by $\delta_{x}: \psi \mapsto \psi(x)$.

Proof. Given $\phi \in \mathcal{C}_{\mathcal{M}}$ and $n \geqslant 2$, using the Definition (62) of $\mathcal{F}_{0 \mathcal{M}}$, the Cauchy-Schwarzinequality, and (36), one has

$$
\begin{aligned}
\left|\mathcal{F}_{0 \mathcal{M}} \phi(x)\right|^{2} & =\left|\frac{(2 \pi)^{-3 / 2}}{m} \int_{\mathcal{M}} e^{-i p x} \phi(p) i_{p}\left(d^{4} p\right)\right|^{2} \\
& \leqslant \frac{(2 \pi)^{-3}}{m^{2}} \int_{\mathcal{M}}\left|q^{0}\right|^{-2} \frac{i_{q}\left(d^{4} q\right)}{q^{0}} \int_{\mathcal{M}}\left|\left(p^{0}\right)^{2} \phi(p)\right|^{2} \frac{i_{p}\left(d^{4} p\right)}{p^{0}} \\
& \leqslant C_{2}{ }^{2}\left\|\left(p_{0}\right)^{2} \phi\right\|^{2} \leqslant C_{2}{ }^{2}\|\phi\|_{\mathcal{M}, n}^{2}
\end{aligned}
$$

with some positive constant $C_{2}=C_{2}(m)$. Setting $\phi=\mathcal{F}_{\mathcal{M} 0} \psi$ for any given $\psi \in \mathcal{C}_{0}$, it follows

$$
|\psi(x)| \leqslant C_{2}\|\phi\|_{\mathcal{M}, n}=C_{2}\|\psi\|_{0, n}
$$

The claim then follows by passing to the completion in $\mathcal{H}_{0, n}$.

\subsection{Existence, Uniqueness, and Causal Structure}

The next theorem is about the well-posedness of the initial value problem corresponding to

(1). For a given $\psi \in \mathcal{C}_{A}$ and a Cauchy surface $\Sigma$, we denote the restriction of $\psi$ to $\Sigma$ by $\left.\psi\right|_{\Sigma} \in \mathcal{C}_{c}^{\infty}\left(\Sigma, \mathbb{C}^{4}\right)$. 
Theorem 2.20 (Initial Value Problem and Support). Let $\Sigma$ be a Cauchy surface and $\chi_{\Sigma} \in$ $\mathcal{C}_{c}^{\infty}\left(\Sigma, \mathbb{C}^{4}\right)$ be given initial data. Then the following is true:

(i) There is a $\psi \in \mathcal{C}_{A}$ such that $\left.\psi\right|_{\Sigma}=\chi_{\Sigma}$ and $\operatorname{supp} \psi \subseteq \operatorname{supp} \chi_{\Sigma}+$ Causal.

(ii) Suppose $\tilde{\psi} \in \mathcal{C}^{\infty}\left(\mathbb{R}^{4}, \mathbb{C}^{4}\right)$ solves the Dirac equation (1) for initial data $\left.\widetilde{\psi}\right|_{\Sigma}=\chi_{\Sigma}$. Then $\widetilde{\psi}=\psi$.

This theorem gives rise to the following definition.

Definition 2.21 (Evolution Operator). Let $\Sigma^{\prime}$ be another Cauchy surface. Given $\chi_{\Sigma}$ with the corresponding $\psi \in \mathcal{C}_{A}$ as above, we define the Dirac evolution from $\Sigma$ to $\Sigma^{\prime}$ by

$$
\mathcal{F}_{\Sigma^{\prime} \Sigma}^{A} \chi_{\Sigma}:=\left.\psi\right|_{\Sigma^{\prime}}
$$

which yields a map $\mathcal{F}_{\Sigma^{\prime} \Sigma}^{A}: \mathcal{C}_{c}^{\infty}\left(\Sigma, \mathbb{C}^{4}\right) \rightarrow \mathcal{C}_{c}^{\infty}\left(\Sigma^{\prime}, \mathbb{C}^{4}\right)$.

As a direct consequence of Theorem 2.20 we infer:

Theorem 2.22 (Unitary Evolution). The map $\mathcal{F}_{\Sigma^{\prime} \Sigma}^{A}: \mathcal{C}_{c}^{\infty}\left(\Sigma, \mathbb{C}^{4}\right) \rightarrow \mathcal{C}_{c}^{\infty}\left(\Sigma^{\prime}, \mathbb{C}^{4}\right)$ extends uniquely to a unitary map $\mathcal{F}_{\Sigma^{\prime} \Sigma}^{A}: \mathcal{H}_{\Sigma} \rightarrow \mathcal{H}_{\Sigma^{\prime}}$.

As discussed in the introduction, there are several different strategies of proof for Theorem 2.20 and 2.22 in the literature. In this work we will give a proof with the help of the just introduced generalized Fourier transforms. We recall that the collected results about these Fourier transforms in Section 2.5 already include a proof of Theorem 2.20 and 2.22 in the case of $A=0$. With a Picard-Lindelöf argument this result can readily be extended to include an external vector potential in the Dirac evolution. We shall use this opportunity to introduce a convenient interaction picture adapted to Cauchy surfaces; see Section 2.7. The main ingredient in the switching from the Schrödinger picture to this interaction picture are again the generalized Fourier transforms. In the interaction picture, the Dirac equation is rephrased in terms of an ordinary differential equation for functions taking values in Sobolev spaces, introduced in Definition 2.18, composed of solutions of the free Dirac equation. The PicardLindelöf theorem then yields existence and uniqueness of solutions, see Lemma 3.9, while regularity of solutions is analyzed with the help of a version of Sobolev's lemma adapted to Cauchy surfaces; see Lemma 3.10. The support properties of the free Dirac evolution and the Picard-Lindelöf iteration imply the support properties of the solutions of the Dirac equation with external potential.

\subsection{An Interaction Picture on Cauchy Surfaces}

As discussed in the previous section it can be useful to switch to an interaction picture in order to treat the interaction with the external potential. For this we introduce a family of Cauchy surfaces $\left(\Sigma_{t}\right)_{t \in \mathbb{R}}$ driven by a family of normal vector fields $\left(\left.v_{t} n_{t}\right|_{\Sigma_{t}}\right)_{t \in \mathbb{R}}$, where $n: \mathbb{R}^{4} \times \mathbb{R} \rightarrow \mathbb{R}^{4}, x \mapsto n_{t}^{\mu}(x)$ and $v: \mathbb{R}^{4} \times \mathbb{R} \rightarrow \mathbb{R}, v:(x, t) \mapsto v_{t}(x)$ are smooth functions. For $x \in \Sigma_{t}$ the vector $n_{t}(x)$ denotes the future-directed unit-normal vector to $\Sigma_{t}$ and $v_{t}$ the 
corresponding normal velocity of the flow of Cauchy surfaces. In particular, given initial values $x_{0} \in \Sigma_{0}$, the solutions of the ODE

$$
\dot{x}_{t}^{\mu}=v_{t}\left(x_{t}\right) n_{t}^{\mu}\left(x_{t}\right), \quad \mu=0,1,2,3,
$$

give rise to trajectories $t \mapsto x_{t}$ with $x_{t} \in \Sigma_{t}$ for all $t \in \mathbb{R}$. Furthermore, we define the set $\Sigma=\left\{(x, t) \in \mathbb{R}^{4} \times \mathbb{R} \mid x \in \Sigma_{t}\right\}$. In case, the following conditions are satisfied:

- $v_{t}(x)>0$ for all $(x, t) \in \Sigma$;

- the projection $F: \Sigma \rightarrow \mathbb{R}^{4},(x, t) \mapsto x$ is a diffeomorphism,

we call $\Sigma$ a future-directed foliation of space-time and define $(y, \tau(y)):=F^{-1}(y)$ for $y \in \mathbb{R}^{4}$ for which

$$
\partial_{\mu} \tau(x)=\left(n_{\tau(x)}\right)_{\mu}(x) v_{\tau(x)}(x)^{-1}
$$

holds. Though defining $n$ and $v$ on $\Sigma$ would suffice, it is sometimes convenient to have them on the whole space $\mathbb{R}^{4} \times \mathbb{R}$. A simple example of a foliation of space-time is given by $\Sigma_{t}=\Sigma+t e_{0}$ for $t \in \mathbb{R}$. The following lemma describes the transition from the Dirac equation in the Schödinger picture to an interaction picture associated to the given family of hypersurfaces and vice versa. It is proven in Section 3.2.3, below.

Theorem 2.23 (Equivalence of the Schrödinger Picture and the Interaction Picture). Consider a future-directed foliation $\Sigma$, the Cauchy surface $\Sigma=\Sigma_{t=0}$, and let $\chi_{\Sigma} \in \mathcal{C}_{\Sigma}$.

(a) Assume that $\psi \in \mathcal{C}_{A}$ fulfills the initial condition $\left.\psi\right|_{\Sigma}=\chi_{\Sigma}$. Define $\phi_{t}=\left.\mathcal{F}_{0 \Sigma_{t}} \psi\right|_{\Sigma_{t}} \in \mathcal{C}_{0}$ for all $t \in \mathbb{R}$. Then the function $\phi: \mathbb{R}^{4} \times \mathbb{R} \rightarrow \mathbb{C}^{4},(x, t) \mapsto \phi(x, t)=\phi_{t}(x)$ is smooth. It fulfills the initial condition

$$
\phi_{0}=\mathcal{F}_{0 \Sigma} \chi_{\Sigma}
$$

and the following evolution equation for all $t \in \mathbb{R}$ and $x \in \mathbb{R}^{4}$ :

$$
i \frac{\partial}{\partial t} \phi_{t}(x)=L_{t} \phi_{t}(x) \quad \text { with } \quad L_{t}:=\mathcal{F}_{0 \Sigma_{t}}\left(v_{t} h_{t} A\right) \mathcal{F}_{\Sigma_{t} 0}: \mathcal{C}_{0} \supset
$$

Here, $\left(v_{t} \hbar_{t} \AA\right): \mathcal{C}_{\Sigma_{t}} \bigcirc$ is understood as a multiplication operator

$$
\left(v_{t} \hbar_{t} A\right) \xi(x)=v_{t}(x) \hbar_{t}(x) \AA(x) \xi(x), \quad \text { for } \xi \in \mathcal{C}_{\Sigma_{t}}, x \in \Sigma_{t} .
$$

Furthermore, there is a compact set $K \subset \mathbb{R}^{4}$ such that for all $t \in \mathbb{R}$ the function $\phi_{t} \in \mathcal{C}_{0}$ is supported in $K+$ Causal. Finally, one has $\psi(x)=\phi(x, \tau(x))$ for all $x \in \mathbb{R}^{4}$.

(b) Conversely, let $\phi: \mathbb{R}^{4} \times \mathbb{R} \rightarrow \mathbb{C}^{4}, \phi(x, t)=\phi_{t}(x)$ be a smooth function, supported in $(K+$ Causal $) \times \mathbb{R}$ for some compact set $K \subseteq \mathbb{R}^{4}$. Assume that $\phi_{t} \in \mathcal{C}_{0}$ for all $t \in \mathbb{R}$, and that $\phi$ fulfills the evolution equation (85) and the initial condition (84). Let

$$
\psi: \mathbb{R}^{4} \rightarrow \mathbb{C}^{4}, \quad \psi(x):=\phi(x, \tau(x))
$$

Then $\psi \in \mathcal{C}_{A}$ and $\left.\psi\right|_{\Sigma}=\chi_{\Sigma}$. Finally, one has $\phi_{t}=\left.\mathcal{F}_{0 \Sigma_{t}} \psi\right|_{\Sigma_{t}}$ for all $t \in \mathbb{R}$. 


\section{Proofs}

In this last section we provide the remaining technical proofs of the claims in Section 2. It is split in two parts. The first part, given in Section 3.1, concerns the generalized Fourier transforms. The second part, given in Section 3.2, concerns the solution theory of the Dirac equation.

\subsection{Generalized Fourier Transforms}

\subsubsection{Properties of the Maps $\mathcal{F}_{3 \mathcal{M}}$ and $\mathcal{F}_{\mathcal{M} 3}$}

The following lemma extends Therorem 2.17.

Lemma 3.1. The maps $\mathcal{F}_{3 \mathcal{M}}$ and $\mathcal{F}_{\mathcal{M} 3}$ are well-defined unitary operators. They are inverse to each other. Furthermore, one has $\mathcal{F}_{3 \mathcal{M}}\left[\mathcal{C}_{\mathcal{M}}\right]=\mathcal{C}_{3}, \mathcal{F}_{\mathcal{M} 3}\left[\mathcal{C}_{3}\right]=\mathcal{C}_{\mathcal{M}}$, and for any $\alpha>0$, $\epsilon>0$, and $n \in \mathbb{N}$ the following bounds hold

$$
\begin{aligned}
\left\|\mathcal{F}_{3 \mathcal{M}} \psi\right\|_{3, \alpha, n-1} & \leqslant C_{3}\|\psi\|_{\mathcal{M}, \alpha, n} & & \text { for } \psi \in \mathcal{C}_{\mathcal{M}}, \\
\left\|\mathcal{F}_{3 \mathcal{M}} \psi\right\|_{3, \alpha, n} & \leqslant C_{4}\|\psi\|_{\mathcal{M}, \alpha+\epsilon, n} & & \text { for } \psi \in \mathcal{C}_{\mathcal{M}}, \\
\left\|\mathcal{F}_{\mathcal{M} 3} \phi\right\|_{\mathcal{M}, \alpha, n} & \leqslant C_{5}\|\phi\|_{3, \alpha, n} & & \text { for } \phi \in \mathcal{C}_{3},
\end{aligned}
$$

with positive constants $C_{3}=C_{3}(n, \alpha, m), C_{4}=C_{4}(n, \alpha, \epsilon, m)$, and $C_{5}=C_{5}(n, m)$.

Proof of Lemma 3.1. We SHOW FIRST that $\mathcal{F}_{3 \mathcal{M}}$ IS AN ISOMETRY. We calculate for $\psi \in$ $\mathcal{H}_{\mathcal{M}}$, using that for $\mathbf{p} \in \mathbb{R}^{3}$, the vectors $\psi\left(p_{+}(\mathbf{p})\right) \in \mathcal{D}_{p_{+}(\mathbf{p})}$ and $\psi\left(p_{-}(\mathbf{p})\right) \in \mathcal{D}_{p_{-}(\mathbf{p})}$ are orthogonal:

$$
\begin{aligned}
\left\|\mathcal{F}_{3 \mathcal{M}} \psi\right\|^{2} & =m^{2} \int_{\mathbb{R}^{3}}\left|\psi\left(p_{+}(\mathbf{p})\right)-\psi\left(p_{-}(\mathbf{p})\right)\right|^{2} \frac{d^{3} \mathbf{p}}{E(\mathbf{p})^{2}}=m^{2} \int_{\mathbb{R}^{3}}\left(\left|\psi\left(p_{+}(\mathbf{p})\right)\right|^{2}+\left|\psi\left(p_{-}(\mathbf{p})\right)\right|^{2}\right) \frac{d^{3} \mathbf{p}}{E(\mathbf{p})^{2}} \\
& =m^{2} \int_{\mathcal{M}}|\psi(p)|^{2} \frac{d^{3} p}{\left(p^{0}\right)^{2}}=\|\psi\|^{2}
\end{aligned}
$$

where we have used equation (36) in the last step.

NeXt, We ShOW That $\mathcal{F}_{3 \mathcal{M}}$ AND $\mathcal{F}_{\mathcal{M} 3}$ ARE INVERSE TO EACH Other. Consider the reflection $r: \mathcal{M} \rightarrow \mathcal{M}, r\left(p^{0}, \mathbf{p}\right)=\left(-p^{0}, \mathbf{p}\right)$. For $\psi \in \mathcal{H}_{\mathcal{M}}$ and $p=\left(p^{0}, \mathbf{p}\right) \in \mathcal{M}$, we get the following, using the definition (23) of $P(p)$.

$$
\mathcal{F}_{\mathcal{M} 3} \mathcal{F}_{3 \mathcal{M}} \psi(p)=\frac{\not p+m}{2} \gamma^{0} \frac{\psi\left(p_{+}(\mathbf{p})\right)-\psi\left(p_{-}(\mathbf{p})\right)}{E(\mathbf{p})}=P(p)(\psi(p)-\psi(r(p)))=\psi(p),
$$

where we have used that $P(p)$ acts as identity on $\mathcal{D}_{p}$ and as zero on $\mathcal{D}_{r(p)}$. Conversely we get for $\phi \in \mathcal{H}_{3}$ :

$$
\mathcal{F}_{3 \mathcal{M}} \mathcal{F}_{\mathcal{M} 3} \phi(\mathbf{p})=P_{+}(\mathbf{p}) \phi(\mathbf{p})+P_{-}(\mathbf{p}) \phi(\mathbf{p})=\phi(\mathbf{p}) .
$$

Because $\mathcal{F}_{3 \mathcal{M}}$ is an isometry, we conclude that $\mathcal{F}_{3 \mathcal{M}}$ and $\mathcal{F}_{\mathcal{M} 3}$ are unitary maps. 
Now, we Show (88), (89) AND $\mathcal{F}_{3 \mathcal{M}}\left[\mathcal{C}_{\mathcal{M}}\right] \subseteq \mathcal{C}_{3}$. Let $\psi \in \mathcal{C}_{\mathcal{M}}$. By definition, $\psi$ has a holomorphic extension $\Psi: \mathcal{M}_{\mathbb{C}} \rightarrow \mathbb{C}^{4}$ that fulfills the bound (31). We extend the reflection $r: \mathcal{M} \rightarrow \mathcal{M}$ to the biholomorphic map $r: \mathcal{M}_{\mathbb{C}} \rightarrow \mathcal{M}_{\mathbb{C}}, r\left(p^{0}, \mathbf{p}\right)=\left(-p^{0}, \mathbf{p}\right)$ and consider the ramification set $Z=\left\{p \in \mathcal{M}_{\mathbb{C}}: p^{0}=0\right\}$ consisting of fixed points of $r$. $Z$ is a complex submanifold of $\mathcal{M}_{\mathbb{C}}$ of codimension 1 ; in particular it has no singular points. The holomorphic map

$$
\chi: \mathcal{M}_{\mathbb{C}} \backslash Z \rightarrow \mathbb{C}^{4}, \quad \chi(p)=m \frac{\Psi(p)-\Psi(r(p))}{p^{0}}
$$

is locally bounded near any point in $Z$, because the numerator $\Psi-\Psi \circ r$ vanishes on $Z$, and the denominator $\mathcal{M}_{\mathbb{C}} \ni p \mapsto p^{0}$ vanishes of first order on $Z$. By Riemann's extension theorem, the map $\chi$ extends to a holomorphic map on the whole set $\mathcal{M}_{\mathbb{C}}$. We denote this extension also by $\chi: \mathcal{M}_{\mathbb{C}} \rightarrow \mathbb{C}^{4}$. Now consider the projection $\pi: \mathcal{M}_{\mathbb{C}} \rightarrow \mathbb{C}^{3}, \pi\left(p^{0}, \mathbf{p}\right)=\mathbf{p}$, and its set of branching points $\pi[Z]=\left\{\mathbf{p} \in \mathbb{C}^{3}: \mathbf{p}^{2}+m^{2}=0\right\}$. Note that $\pi^{-1}[\pi(p)]=\{p, r(p)\}$ holds for any $p \in \mathcal{M}_{\mathbb{C}}$, and that $\pi[Z]$ is also a submanifold of $\mathbb{C}^{3}$ of complex codimension 1 ; in particular it has also no singular points. Since $\chi \circ r=\chi$, there is a map $\Phi: \mathbb{C}^{3} \rightarrow \mathbb{C}^{4}$ such that $\Phi \circ \pi=\chi$. Obviously $\Phi$ is holomorphic outside the branching points, i.e. on $\mathbb{C}^{3} \backslash \pi[Z]$, and it is locally bounded near any branching point $\mathbf{p} \in \pi[Z]$. Using Riemann's extension theorem again, we see that $\Phi$ is holomorphic on its whole domain $\mathbb{C}^{3}$. Comparing definitions (59) and (94), we see that $\Phi: \mathbb{C}^{3} \rightarrow \mathbb{C}^{4}$ is a holomorphic extension of $\phi:=\mathcal{F}_{3 \mathcal{M}} \psi: \mathbb{R}^{3} \rightarrow \mathbb{C}^{4}$. To finish the proof of $\mathcal{F}_{3 \mathcal{M}} \psi \in \mathcal{C}_{3}$, it remains to show that $\Phi$ fulfills the bound (89); recall the definition $(39) /(40)$. Take $\alpha>0$ such $(31) /(32)$ holds. Let $n \in \mathbb{N}$. Using the definition (94) of $\chi$, we get the following for all $p \in \mathcal{M}_{\mathbb{C}} \backslash Z$ :

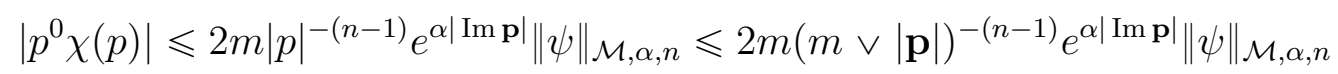

For the last step, we have used $m \leqslant|p|$ from (202) in Lemma A.1 and $|\mathbf{p}| \leqslant|p|$. We distinguish two cases:

Case 1, "locations far from the ramification set": $\left|p^{0}\right| \geqslant m / 12$. On the one hand, (95) implies in this case the following.

$$
|\chi(p)| \leqslant 24(m \vee|\mathbf{p}|)^{-(n-1)} e^{\alpha|\operatorname{Im} \mathbf{p}|\|\psi\|_{\mathcal{M}, \alpha, n} .}
$$

On the other hand, from (95) and inequality (203) from Lemma A.1, we get for any given $\epsilon>0$

$$
|\chi(p)| \leqslant \frac{2 m(m \vee \mathbf{p})^{1-n}}{\frac{m}{12} \vee\left|p^{0}\right|} e^{\alpha|\operatorname{Im} \mathbf{p}|}\|\psi\|_{\mathcal{M}, \alpha, n} \leqslant C_{6}(m \vee|\mathbf{p}|)^{-n} e^{(\alpha+\epsilon)|\operatorname{Im} \mathbf{p}|\|\psi\|_{\mathcal{M}, \alpha, n}}
$$

where $C_{6}=C_{6}(\epsilon, m):=2 m C_{16}$.

Case 2, "locations close to the ramification set": $\left|p^{0}\right|<m / 12$. The key to deal with this case is provided by the following lemma, which uses the geometric structure of $\mathcal{M}_{\mathbb{C}}$ close to the ramification set. The intuitive idea behind it relies on the fact that the three components of the differential form $d \mathbf{p}$ on $\mathcal{M}_{\mathbb{C}}$ become linearly dependent on the ramification set $Z$, while the form $d p^{0}$ on $\mathcal{M}_{\mathbb{C}}$ does not vanish there. Consequently, close to any ramification point, one can find a complex direction tangential to $\mathcal{M}_{\mathbb{C}}$ such that $p^{0}$ varies considerably in that direction, while $d \mathbf{p}$ does not vary too much in the same direction. This vague idea is made precise and quantitative in the following lemma. 
Lemma 3.2. For every $p=\left(p^{0}, \mathbf{p}\right) \in \mathcal{M}_{\mathbb{C}}$ with $\left|p^{0}\right| \leqslant m / 12$, there is a holomorphic map

$$
k=\left(k^{0}, \mathbf{k}\right): \bar{\Delta} \rightarrow \mathcal{M}_{\mathbb{C}}
$$

defined on the closed unit disc $\bar{\Delta}=\{t \in \mathbb{C}:|t| \leqslant 1\}$ with

$$
\begin{gathered}
k(0)=p, \\
|\mathbf{k}(t)-\mathbf{p}| \leqslant m / 6 \quad \text { for } t \in \bar{\Delta}, \\
\left|k^{0}(t)\right| \geqslant m / 12 \quad \text { for } t \in \partial \Delta,
\end{gathered}
$$

where $\partial \Delta=\{t \in \mathbb{C}:|t|=1\}$ denotes the unit circle.

This lemma is also proven in the appendix. In the following estimates (102) and (103), we apply the function $k$ from this lemma together with the maximum principle for holomorphic functions. Then, the inequalities (96) and (97), respectively, are used with $p$ replaced by $k(t)$ with $t \in \partial \Delta$. The hypothesis $\left|k^{0}(t)\right| \geqslant m / 12$ of these two inequalities is verified by (101). Using also (100) we get

$$
\begin{aligned}
|\Phi(\mathbf{p})|=|\chi(p)| & \leqslant \sup _{t \in \partial \Delta}|\chi(k(t))| \leqslant 24 \sup _{t \in \partial \Delta}(m \vee|\mathbf{k}(t)|)^{-(n-1)} e^{\alpha|\operatorname{Im} \mathbf{k}(t)|}\|\psi\|_{\mathcal{M}, \alpha, n} \\
& \leqslant C_{3}(m \vee|\mathbf{p}|)^{-(n-1)} e^{\alpha|\operatorname{Im} \mathbf{p}|\|\psi\|_{\mathcal{M}, \alpha, n},} \\
|\Phi(\mathbf{p})|=|\chi(p)| & \leqslant C_{6} \sup _{t \in \partial \Delta}(m \vee|\mathbf{k}(t)|)^{-n} e^{(\alpha+\epsilon)|\operatorname{Im} \mathbf{k}(t)|}\|\psi\|_{\mathcal{M}, \alpha, n} \\
& \leqslant C_{4}(m \vee|\mathbf{p}|)^{-n} e^{(\alpha+\epsilon)|\operatorname{Im} \mathbf{p}|\|\psi\|_{\mathcal{M}, \alpha, n}}
\end{aligned}
$$

with constants $C_{3}=C_{3}(n, \alpha, m)>24$ and $C_{4}=C_{4}(n, \alpha, \epsilon, m)>C_{6}$. This proves the bounds (88), (89) and thus, the claim $\mathcal{F}_{3 \mathcal{M}} \psi \in \mathcal{C}_{3}$, which yields $\mathcal{F}_{3 \mathcal{M}}\left[\mathcal{C}_{\mathcal{M}}\right] \subseteq \mathcal{C}_{3}$.

It Remains to Show the Bound (90) AND $\mathcal{F}_{\mathcal{M} 3}\left[\mathcal{C}_{3}\right] \subseteq \mathcal{C}_{\mathcal{M}}$. Given $\phi \in \mathcal{C}_{3}$, we have a holomorphic continuation $\Phi: \mathbb{C}^{3} \rightarrow \mathbb{C}^{4}$ that fulfills the bound (39)/(40). Then $\psi:=\mathcal{F}_{\mathcal{M} 3} \phi$ has the holomorphic continuation

$$
\Psi: \mathcal{M}_{\mathbb{C}} \rightarrow \mathbb{C}^{4}, \quad \Psi(p)=\frac{\not p+m}{2 m} \gamma^{0} \Phi(\mathbf{p}) \quad \text { for } p=\left(p^{0}, \mathbf{p}\right) \in \mathcal{M}_{\mathbb{C}} .
$$

For the matrix norm $\|\cdot\|$ on $\mathbb{C}^{4 \times 4}$ associated to the euclidean norm on $\mathbb{C}^{4}$, one observes $\|\not p\|=|p|$ and $\left\|\gamma^{0}\right\|=1$. The following estimate uses these two equalities in the first step, the first inequality in (202) in the second step, formula (40) in the third step, and the second inequality in (202) in the last step.

$$
\begin{aligned}
|\Psi(p)| & \leqslant \frac{|p|+m}{2 m}|\Phi(\mathbf{p})| \leqslant \frac{|p|}{m}|\Phi(\mathbf{p})| \leqslant \frac{|p|}{m} \mid(m \vee|\mathbf{p}|)^{-n} e^{\alpha|\operatorname{Im} \mathbf{p}|}\|\phi\|_{3, \alpha, n} \\
& \leqslant \frac{3^{n / 2}}{m}|p|^{-(n-1)} e^{\alpha|\operatorname{Im} \mathbf{p}|}\|\phi\|_{3, \alpha, n} .
\end{aligned}
$$

Using the definition (32) of $\|\psi\|_{\mathcal{M}, \alpha, n}$, this shows the bound (90) and thus $\mathcal{F}_{\mathcal{M} 3} \phi \in \mathcal{C}_{\mathcal{M}}$.

We summarize: We have proven $\mathcal{F}_{3 \mathcal{M}}\left[\mathcal{C}_{\mathcal{M}}\right] \subseteq \mathcal{C}_{3}$ and $\mathcal{F}_{\mathcal{M} 3}\left[\mathcal{C}_{3}\right] \subseteq \mathcal{C}_{\mathcal{M}}$. Because $\mathcal{F}_{3 \mathcal{M}}$ and $\mathcal{F}_{3 \mathcal{M}}$ are inverse to each other, this also proves the claims $\mathcal{F}_{3 \mathcal{M}}\left[\mathcal{C}_{\mathcal{M}}\right]=\mathcal{C}_{3}$ and $\mathcal{F}_{\mathcal{M} 3}\left[\mathcal{C}_{3}\right]=$ $\mathcal{C}_{\mathcal{M}}$ 
Finally, one observes the following corollary to Lemma 3.1.

Corollary 3.3. $\mathcal{C}_{\mathcal{M}}$ is dense in $\mathcal{H}_{\mathcal{M}}$.

Proof. By Lemma 2.9, $\mathcal{C}_{3}$ is dense in $\mathcal{H}_{3}$. Furthermore, by Lemma 3.1, the map $\mathcal{F}_{\mathcal{M} 3}: \mathcal{H}_{3} \rightarrow$ $\mathcal{H}_{\mathcal{M}}$ is unitary and maps $\mathcal{C}_{3}$ onto $\mathcal{C}_{\mathcal{M}}$. The claim follows.

\subsubsection{Properties of the Maps $\mathcal{F}_{\mathcal{M} \Sigma}, \mathcal{F}_{\Sigma 0}$ and $\mathcal{F}_{0 \mathcal{M}}$}

In this section, we prove three technical, but important lemmas. The first one, Lemma 3.4, deals with the generalized Fourier transformation $\mathcal{F}_{\mathcal{M} \Sigma}: \mathcal{C}_{\Sigma} \rightarrow \mathcal{C}_{\mathcal{M}}$ from wave functions on $\Sigma$ to wave functions on the mass shell. It relies on Paley-Wiener-like bounds: Support properties in physical space are translated to growth rates in imaginary directions in the complexified mass shell. The second lemma, Lemma 3.5, deals with the maps $\mathcal{F}_{0 \mathcal{M}}: \mathcal{C}_{\mathcal{M}} \rightarrow \mathcal{C}_{0}$ and $\mathcal{F}_{\Sigma 0}: \mathcal{C}_{0} \rightarrow \mathcal{C}_{\Sigma}$. Here, the point is to translate growth rates in imaginary directions in the complexified mass shell back to support properties in physical space, using the classical Paley-Wiener theorem. Finally, the third lemma, Lemma 3.6, is about compositions of these three maps. In particular, it controls the support of a solution of the free Dirac equation with given initial data on a Cauchy surface.

Recall the definitions of $\mathcal{S}(K, v), \mathcal{C}_{\Sigma}(K)$, and $\|\cdot\|_{\Sigma, K, n}$ given in the first paragraph of Section 2.5.1. The following lemma slightly extends Theorem 3.4.

Lemma 3.4 (Paley-Wiener Bounds for Cauchy Surfaces). For any Cauchy surface $\Sigma$ the map $\mathcal{F}_{\mathcal{M} \Sigma}: \mathcal{C}_{\Sigma} \rightarrow \mathcal{C}_{\mathcal{M}}$ is well-defined. More precisely, let $K \subset \mathbb{R}^{4}$ be compact, $0 \leqslant V<1$, $\Sigma \in \mathcal{S}(K, V), \psi \in \mathcal{C}_{\Sigma}(K), \alpha$ be a positive number such that $\alpha>\sqrt{2} \sup _{x \in K}|x|$, and $n \in \mathbb{N}$. Then

$$
\left\|\mathcal{F}_{\mathcal{M} \Sigma} \psi\right\|_{\mathcal{M}, \alpha, n} \leqslant C_{1}\|\psi\|_{\Sigma, K, n}
$$

holds for some some positive constant $C_{1}=C_{1}(K, V, n, \alpha, m)$.

Proof. The wave function $\psi \in \mathcal{C}_{\Sigma}(K)$ is supported on the compact set $K \cap \Sigma$. We consider the following integral:

$$
\Psi(p):=\frac{\not p+m}{2 m}(2 \pi)^{-3 / 2} \int_{K \cap \Sigma} e^{i p x} i_{\gamma}\left(d^{4} x\right) \psi(x) \quad \text { for } p \in \mathcal{M}_{\mathbb{C}},
$$

which coincides with $\left(\mathcal{F}_{\mathcal{M} \Sigma} \psi\right)(p)$ for $p \in \mathcal{M}$; see $(61)$. Because $(\not p-m)(\not p+m)=p^{2}-m^{2}=0$ holds for $p \in \mathcal{M}_{\mathbb{C}}$, one has $(\not p-m) \Psi(p)=0$ for these $p$. In particular, $\left(\mathcal{F}_{\mathcal{M} \Sigma} \psi\right)(p) \in \mathcal{D}_{p}$ holds for $p \in \mathcal{M}$.

OUR NeXt GOAL IS TO EStimate $\Psi(p)$ FOR $p \in \mathcal{M}_{\mathbb{C}}$. For this we intend to use the decay of the Fourier transform for smooth compactly supported functions in $\mathbb{R}^{3}$, at least for sufficiently large $|\operatorname{Re} p|$. Therefore, we shall employ a projection $\pi_{\hat{q}}^{\Sigma}: \Sigma \rightarrow \mathbb{R}^{3} \simeq \Sigma^{0}=$ $\{0\} \times \mathbb{R}^{3}$ in some time-like direction $\hat{q} \in \mathbb{R}^{4},|\hat{q}|=1$, which fulfills:

(a) $\hat{q}$ is transversal to $\Sigma^{0}$ and to every tangent space of $\Sigma$. 
(b) $\hat{q} \in(\operatorname{Re} p)^{\perp}:=\left\{y \in \mathbb{R}^{4} \mid \operatorname{Re} p_{\mu} y^{\mu}=0\right\}$ in order to have

$$
\exp \left(i \operatorname{Re} p_{\mu} x^{\mu}\right)=\exp \left(i \operatorname{Re} p_{\mu}\left(s \hat{q}^{\mu}+x^{\mu}\right)\right) \quad \text { for any } s \in \mathbb{R} \text {. }
$$

First, we focus on condition (a). Note that light-like vectors fulfill (a). By definition of $\mathcal{S}(K, V)$, the set

$$
N=N(K, V):=\left\{\hat{q} \in \mathbb{R}^{4}|| \hat{q} \mid=1, \hat{q} \text { is transversal to } \Sigma^{0} \text { and to all } \Sigma \in \mathcal{S}(K, V)\right\}
$$

is a neighborhood of the set of light-like vectors in the unit sphere. Therefore, we can choose $\epsilon=\epsilon(K, V)>0$ sufficiently small such that

$$
E=E(K, V):=\left\{k \in \mathbb{R}^{4}|| k|=1,| k^{2} \mid \leqslant \epsilon\right\} \subseteq N .
$$

Note that $E$ is compact. For $\Sigma \in \mathcal{S}(K, V), \hat{q} \in E$, and $x \in \Sigma$ we define the projection

$$
\left(0, \pi_{\hat{q}}^{\Sigma}(x)\right)=\left(s \hat{q}^{\mu}+x^{\mu}\right)_{\mu=0,1,2,3} \in \Sigma^{0}
$$

with $s=-x^{0} / \hat{q}^{0}$. Note that $\pi_{\hat{q}}^{\Sigma}$ is a diffeomorphism from $\Sigma$ to $\mathbb{R}^{3}$, and $\pi_{\hat{q}}^{\Sigma}$ and all its derivatives depend continuously on $\hat{q} \in E$.

Second, we focus on condition (b). To fulfill this condition the direction $\hat{q}$ must be chosen to depend on $p \in \mathcal{M}_{\mathbb{C}}$, i.e., $\hat{q}=\hat{q}(p)$. Therefore, for $p \in \mathbb{C}^{4}$ with $\operatorname{Re} \mathbf{p} \neq 0$ we define

$$
\hat{q}^{\mu}(p):=\frac{q^{\mu}(p)}{|q(p)|} \in(\operatorname{Re} p)^{\perp} \quad \text { with } \quad q(p):=\left(|\operatorname{Re} \mathbf{p}|,\left(\operatorname{Re} p^{0}\right) \frac{\operatorname{Re} \mathbf{p}}{|\operatorname{Re} \mathbf{p}|}\right) .
$$

However, for this choice of $\hat{q}(p)$ it may occur that condition (a) is violated. Therefore we restrict $p$ to the following set

$$
I=I(K, V):=\left\{\left.p \in \mathcal{M}_{\mathbb{C}}|| \operatorname{Im} p\right|^{2}+m^{2} \leqslant \epsilon|\operatorname{Re} p|^{2}, \operatorname{Re} \mathbf{p} \neq 0\right\} .
$$

Indeed, we have $\hat{q}[I] \subseteq E$ as the following argument shows. Let $p \in I$. We have $p^{2}-m^{2}=0$, and thus

$$
(\operatorname{Re} p)^{2}=(\operatorname{Im} p)^{2}+m^{2} \leqslant|\operatorname{Im} p|^{2}+m^{2} \leqslant \epsilon|\operatorname{Re} p|^{2}
$$

In other words

$$
\frac{\operatorname{Re} p}{|\operatorname{Re} p|} \in E
$$

Furthermore, we have $q(p)^{2}=-(\operatorname{Re} p)^{2}$ and $|q(p)|=|\operatorname{Re} p|$, which imply

$$
\frac{\operatorname{Re} p}{|\operatorname{Re} p|} \in E \Leftrightarrow \hat{q}(p) \in E
$$

Together with (115) this shows $\hat{q}(p) \in E$. In consequence, $\hat{q}(p)$ fulfills conditions (a) and (b) for all $p \in I$. 
IN THE NEXT STEP, WE PROVIDE A BOUND ON $\Psi(p)$ DEFINED IN (107) IN THE CASE OF $p \in I$. Using the transformation $\mathbf{y}=\pi_{\hat{q}(p)}^{\Sigma}(x)$, which fulfills $\operatorname{Re} p x=-\operatorname{Re} \mathbf{p} \cdot \mathbf{y}$ by construction, we get

$$
\int_{x \in K \cap \Sigma} e^{i p x} i_{\gamma}\left(d^{4} x\right) \psi(x)=\int_{\mathbf{y} \in \pi_{\hat{q}(p)}^{\Sigma}[K \cap \Sigma]} e^{-i \operatorname{Re} \mathbf{p} \cdot \mathbf{y}} f_{p}(\mathbf{y}) d^{3} \mathbf{y}
$$

for

$$
f_{p}(\mathbf{y}):=\exp \left(-\operatorname{Im} p_{\mu}\left[\left(\pi_{\hat{q}(p)}^{\Sigma}\right)^{-1}(\mathbf{y})\right]^{\mu}\right) g_{\hat{q}(p)}(\mathbf{y})
$$

where

$$
g_{\hat{q}}(\mathbf{y}) d^{3} \mathbf{y}:=\left(\left(\left(\pi_{\hat{q}}^{\Sigma}\right)^{-1}\right)^{*}\left(i_{\gamma}\left(d^{4} x\right) \psi(x)\right)\right.
$$

denotes the pull-back of $i_{\gamma}\left(d^{4} x\right) \psi(x)$ w.r.t. $\left(\pi_{\hat{q}}^{\Sigma}\right)^{-1}$.

Thanks to compactness of $E$ and $K$ and continuity in $\hat{q} \in E$, for all multi-indices $\beta \in \mathbb{N}_{0}^{3}$ with $|\beta| \leqslant n$, the following holds, with the differentiation operators $D^{\beta}$ acting on the variable y:

$$
\sup _{\Sigma \in \mathcal{S}(K, V)} \sup _{\hat{q} \in E} \sup _{\mathbf{y} \in \pi_{\hat{q}}[K \cap \Sigma]}\left|\left(D^{\beta}\left(\pi_{\hat{q}}^{\Sigma}\right)^{-1}\right)(\mathbf{y})\right|<\infty
$$

and hence,

$$
\sup _{\Sigma \in \mathcal{S}(K, V)} \sup _{\hat{q} \in E} \sup _{\mathbf{y} \in \pi_{\hat{q}}^{\Sigma}[K \cap \Sigma]}\left|D^{\beta} g_{\hat{q}}(\mathbf{y})\right|<C_{7}\|\psi\|_{\Sigma, K, n},
$$

with a constant $C_{7}=C_{7}(K, V, n)$. For any given $\delta>0$, taking

$$
\widetilde{\alpha}=\widetilde{\alpha}(\delta, K):=2 \delta+\sup _{x \in K}|x|
$$

we know

$$
\begin{aligned}
& \sup _{\Sigma \in \mathcal{S}(K, V)} \sup _{p \in I} \sup _{\mathbf{y} \in \pi_{\hat{q}(p)}^{\Sigma}[K \cap \Sigma]} e^{(2 \delta-\widetilde{\alpha})|\operatorname{Im} p|} \exp \left(-\operatorname{Im} p_{\mu}\left[\left(\pi_{\hat{q}(p)}\right)^{-1}(\mathbf{y})\right]^{\mu}\right) \\
& =\sup _{\Sigma \in \mathcal{S}(K, V)} \sup _{p \in I} \sup _{x \in K \cap \Sigma} e^{(2 \delta-\widetilde{\alpha})|\operatorname{Im} p|-x \operatorname{Im} p} \leqslant 1
\end{aligned}
$$

We obtain:

$\max _{|\beta| \leqslant n} \sup _{\Sigma \in \mathcal{S}(K, V)} \sup _{p \in I} \sup _{\mathbf{y} \in \pi_{\tilde{q}(p)}^{\Sigma}[K \cap \Sigma]}(m+|\operatorname{Im} p|)^{-n} e^{(2 \delta-\widetilde{\alpha})|\operatorname{Im} p|}\left|D^{\beta} \exp \left(-\operatorname{Im} p_{\mu}\left[\left(\pi_{\hat{q}(p)}^{\Sigma}\right)^{-1}(\mathbf{y})\right]^{\mu}\right)\right|<\infty$.

To see this, one expresses the iterated derivatives $D^{\beta} \ldots$ with the chain rule and uses the bound (120) for the inner derivatives and compactness of the set $\left\{\left(\hat{q}, \pi_{\hat{q}}^{\Sigma}(x)\right) \mid \hat{q} \in E, x \in\right.$ $K \cap \Sigma, \Sigma \in \mathcal{S}(K, V)\}$. Combining the bound (125) with the bound (121) yields

$$
\max _{|\beta| \leqslant n} \sup _{\Sigma \in \mathcal{S}(K, V)} \sup _{p \in I} \sup _{\mathbf{y} \in \pi_{\hat{q}(p)}^{\Sigma}[K \cap \Sigma]}(m+|\operatorname{Im} p|)^{-n} e^{(2 \delta-\widetilde{\alpha})|\operatorname{Im} p|}\left|D^{\beta} f_{p}(\mathbf{y})\right| \leqslant C_{8}\|\psi\|_{\Sigma, K, n},
$$


and, a little weaker,

$$
\max _{|\beta| \leqslant n} \sup _{\Sigma \in \mathcal{S}(K, V)} \sup _{p \in I} \sup _{\mathbf{y} \in \pi_{\tilde{q}(p)}^{\Sigma}[K \cap \Sigma]} e^{(\delta-\widetilde{\alpha})|\operatorname{Im} p|}\left|D^{\beta} f_{p}(\mathbf{y})\right| \leqslant C_{9}\|\psi\|_{\Sigma, K, n}
$$

for some constants $C_{8}=C_{8}(K, V, n, \delta, m)$ and $C_{9}=C_{9}(K, V, n, \delta, m)$.

Using compactness again, there is $R>0$ such that

$$
\bigcup_{p \in I} \operatorname{supp} f_{p} \subseteq \bigcup_{\substack{q \in E \\ \Sigma \in \mathcal{S}(K, V)}} \pi_{\hat{q}}^{\Sigma}[K \cap \Sigma] \subseteq \overline{B_{R}^{3}(0)},
$$

where $\overline{B_{R}^{3}(0)}$ denotes the closed 3-dimensional ball with radius $R$ around 0 .

The bound (127) of the derivatives together with the boundedness (128) yield the following bound for the Fourier transform for some constant $C_{10}=C_{10}(K, V, n, \delta, m)$.

$$
\left|\widehat{f}_{p}(\mathbf{k})\right| \leqslant C_{10} \frac{e^{(\widetilde{\alpha}-\delta)|\operatorname{Im} p|}}{(m+|\mathbf{k}|)^{n}}\|\psi\|_{\Sigma, K, n}, \quad \forall \mathbf{k} \in \mathbb{R}^{3}, p \in I .
$$

In the special case $\mathbf{k}=\operatorname{Re} \mathbf{p}$ it tells us the following for all $p \in I$ and $n \in \mathbb{N}_{0}$ :

$$
\begin{aligned}
\left|\int_{\mathbf{y} \in \pi_{\tilde{q}(p)}^{\Sigma}[K \cap \Sigma]} e^{-i \operatorname{Re} \mathbf{p} \cdot \mathbf{y}} f_{p}(\mathbf{y}) d^{3} \mathbf{y}\right| & \leqslant C_{10} \frac{e^{(\widetilde{\alpha}-\delta)|\operatorname{Im} p|}}{(m+|\operatorname{Re} \mathbf{p}|)^{n}}\|\psi\|_{\Sigma, K, n} \\
& \leqslant C_{11} \frac{e^{\widetilde{\alpha}|\operatorname{Im} p|}}{(m+|\mathbf{p}|)^{n}}\|\psi\|_{\Sigma, K, n} \leqslant C_{12}|p|^{-n} e^{\widetilde{\alpha}|\operatorname{Im} p|}\|\psi\|_{\Sigma, K, n}
\end{aligned}
$$

with some constants $C_{11}=C_{11}(K, V, n, \delta, m)$ and $C_{12}=C_{12}(K, V, n, m)$, where in the last step we have used bound (202) given in Lemma A.1. Combining this with (117) and using $\|p+m\| \leqslant|p|+m \leqslant 2|p|$ yields

$$
|\Psi(p)|=\left|\frac{p+m}{2 m}(2 \pi)^{-3 / 2} \int_{K \cap \Sigma} e^{i p x} i_{\gamma}\left(d^{4} x\right) \psi(x)\right| \leqslant C_{13}|p|^{-(n-1)} e^{\widetilde{\alpha}|\operatorname{Im} p|}\|\psi\|_{\Sigma, K, n} \quad \text { for } p \in I
$$

with some sufficiently large constant $C_{13}=C_{13}(K, V, n, \delta, m)$ and $\Psi$ being defined in (107).

NeXt, We EXAMine the EASIER CASE $p \in \mathcal{M}_{\mathbb{C}} \backslash I$. By definition (113) of $I$, we have $|\operatorname{Im} p|^{2}+m^{2}>\epsilon|\operatorname{Re} p|^{2}$, called "case A", or Re $\mathbf{p}=0$, called "case B". In case A, $|p| \leqslant$ $C_{14}(m+|\operatorname{Im} p|)$ holds with some constant $C_{14}=C_{14}(\epsilon)$. In case B, the same bound holds when $C_{14}$ is chosen sufficiently large. Indeed: $\operatorname{Re} \mathbf{p}=0$ and $p^{2}=m^{2}$ imply $|\operatorname{Re} p|^{2}=$ $\left(\operatorname{Re} p^{0}\right)^{2}=(\operatorname{Re} p)^{2}=(\operatorname{Im} p)^{2}+m^{2} \leqslant|\operatorname{Im} p|^{2}+m^{2}$, and hence, $|p|^{2} \leqslant 2|\operatorname{Im} p|^{2}+m^{2}$. Taking $\widetilde{\alpha}=2 \delta+\sup _{x \in K}|x|$ as in (122) and a sufficiently large constant $C_{15}=C_{15}(K, V, n, \delta, m)$, we conclude the following for $p \in \mathcal{M}_{\mathbb{C}} \backslash I, n \in \mathbb{N}$ :

$$
\begin{aligned}
|\Psi(p)| & \leqslant C_{15}|p| e^{(\widetilde{\alpha}-2 \delta)|\operatorname{Im} p|}\|\psi\|_{\Sigma, K, n} \leqslant C_{15}|p| e^{2 \delta\left(m-|p| / C_{14}\right)} e^{\widetilde{\alpha}|\operatorname{Im} p|}\|\psi\|_{\Sigma, K, n} \\
& \leqslant C_{13}|p|^{-(n-1)} e^{\widetilde{\alpha}|\operatorname{Im} p|}\|\psi\|_{\Sigma, K, n}
\end{aligned}
$$


where the constant $C_{13}(K, V, n, \delta, m)$, which was also used in (132) in a different way, needs to be taken large enough. In the first inequality in (134), we used the definition (107) of $\Psi(p)$ and again the bound $\|\not p+m\| \leqslant 2|p|$ together with the estimate $\left|e^{i p x}\right| \leqslant e^{(\tilde{\alpha}-2 \delta)|\operatorname{Im} p|}$ for $p \in \mathcal{M}_{\mathbb{C}} \backslash I$ and $x \in K$. Combining (134) and (132) we have shown

$$
|\Psi(p)| \leqslant C_{13}|p|^{-(n-1)} e^{\widetilde{\alpha}|\operatorname{Im} p|}\|\psi\|_{\Sigma, K, n} \leqslant C_{13}|p|^{-(n-1)} e^{\sqrt{2} \widetilde{\alpha}|\operatorname{Im} \mathbf{p}|}\|\psi\|_{\Sigma, K, n} \quad \text { for all } p \in \mathcal{M}_{\mathbb{C}}
$$

where we have used (201) from Lemma A.1 in the last step. Because $\Psi: \mathcal{M}_{\mathbb{C}} \rightarrow \mathbb{C}^{4}$ is holomorphic, we can rewrite this as

$$
\left\|\mathcal{F}_{\mathcal{M} \Sigma} \psi\right\|_{\mathcal{M}, \sqrt{2} \widetilde{\alpha}, n} \leqslant C_{13}\|\Psi\|_{\Sigma, K, n}
$$

with the norm $\|\cdot\|_{\mathcal{M}, \sqrt{2} \widetilde{\alpha}, n}$ being defined in (32). We now take a specific $\delta>0$ depending on the given $\alpha>\sqrt{2} \sup _{x \in K}\|x\|$ such that the equation

$$
\alpha=\sqrt{2} \widetilde{\alpha}(\delta, K)
$$

holds. This concludes the proof of (106). Hence, $\mathcal{F}_{\mathcal{M} \Sigma}: \mathcal{C}_{\Sigma} \rightarrow \mathcal{C}_{\mathcal{M}}$ is well-defined which proves the claim.

Lemma 3.5. The maps $\mathcal{F}_{0 \mathcal{M}}: \mathcal{C}_{\mathcal{M}} \rightarrow \mathcal{C}_{0}$ and $\mathcal{F}_{\Sigma 0}: \mathcal{C}_{0} \rightarrow \mathcal{C}_{\Sigma}$ are well-defined. For any $\psi \in \mathcal{C}_{\mathcal{M}}$ and $\alpha>0$ such that $\|\psi\|_{\mathcal{M}, \alpha, n}<\infty$ holds for all $n \in \mathbb{N}$, the function $\mathcal{F}_{0 \mathcal{M}} \psi$ is supported in $\{0\} \times \overline{B_{\alpha}^{3}(0)}+$ Causal.

Proof. First, we Show that $\mathcal{F}_{0 \mathcal{M}}: \mathcal{C}_{\mathcal{M}} \rightarrow \mathcal{C}_{0}$ Is Well-Defined. Let $\psi \in \mathcal{C}_{\mathcal{M}}, \Psi: \mathcal{M}_{\mathbb{C}} \rightarrow$ $\mathbb{C}^{4}$ be the holomorphic extension of $\psi$ to $\mathcal{M}_{\mathbb{C}}$, and take $\alpha>0$ such that for all $n \in \mathbb{N}$ the bound $\|\psi\|_{\mathcal{M}, \alpha, n}<\infty$ holds. Because $|\psi(p)|$ tends to 0 as $|p| \rightarrow \infty, p \in \mathcal{M}$, faster than any power of $|p|$,

$$
\mathcal{F}_{0 \mathcal{M}} \psi(x)=\frac{(2 \pi)^{-3 / 2}}{m} \int_{\mathcal{M}} e^{-i p x} \psi(p) i_{p}\left(d^{4} p\right)
$$

depends smoothly on $x \in \mathbb{R}^{4}$. Furthermore, $\mathcal{F}_{0 \mathcal{M}} \psi$ solves the free Dirac equation because the fact $\psi(p) \in \mathcal{D}_{p}$ for any $p \in \mathcal{M}$ implies

$$
(i \not \supset-m) \mathcal{F}_{0 \mathcal{M}} \psi(x)=\frac{(2 \pi)^{-3 / 2}}{m} \int_{\mathcal{M}} e^{-i p x}(\not p-m) \psi(p) i_{p}\left(d^{4} p\right)=0 \quad \text { for } x \in \mathbb{R}^{4} .
$$

Given $t \in \mathbb{R}$, we introduce the time-shifted version

$$
\Psi_{t}(p):=e^{-i p_{0} t} \Psi(p), \quad p \in \mathcal{M}_{\mathbb{C}}
$$

The restriction of this holomorphic map to $\mathcal{M}$ is denoted by $\psi_{t}$. We observe the following for $p=\left(p^{0}, \mathbf{p}\right) \in \mathcal{M}_{\mathbb{C}}$, using the bound $\left|\operatorname{Im} p^{0}\right| \leqslant|\operatorname{Im} \mathbf{p}|$ from $(200)$ :

$$
\left|\Psi_{t}(p)\right| \leqslant e^{\left|\operatorname{Im} p_{0}\right||t|}|\Psi(p)| \leqslant e^{|\operatorname{Im} \mathbf{p}||t|}|\Psi(p)|
$$

We get

$$
\left\|\psi_{t}\right\|_{\mathcal{M}, \alpha+|t|, n} \leqslant\|\psi\|_{\mathcal{M}, \alpha, n}, \quad(n \in \mathbb{N})
$$


recall the definition of $\|\cdot\|_{\mathcal{M}, \alpha, n}$ from (32). Then the bound (88) implies

$$
\left\|\mathcal{F}_{3 \mathcal{M}} \psi_{t}\right\|_{3, \alpha+|t|, n-1} \leqslant C_{3}\left\|\psi_{t}\right\|_{\mathcal{M}, \alpha+|t|, n} \leqslant C_{3}\|\psi\|_{\mathcal{M}, \alpha, n}<\infty, \quad(n \in \mathbb{N}) .
$$

Using this, the classical Paley-Wiener Theorem [RS81, Theorem IX.11] implies that the inverse Fourier transform

$$
\mathbb{R}^{3} \ni \mathbf{x} \mapsto(2 \pi)^{-3 / 2} \int_{\mathbb{R}^{3}} e^{i \mathbf{p x}} \mathcal{F}_{3 \mathcal{M}} \psi_{t}(\mathbf{p}) d^{3} \mathbf{p}
$$

is supported in the ball $\overline{B_{\alpha+|t|}^{3}(0)}$. Taking $x=(t, \mathbf{x})$ we compute

$$
\begin{aligned}
& (2 \pi)^{-3 / 2} \int_{\mathbb{R}^{3}} e^{i \mathbf{p x}} \mathcal{F}_{3 \mathcal{M}} \psi_{t}(\mathbf{p}) d^{3} \mathbf{p}=\frac{(2 \pi)^{-3 / 2}}{m} \int_{\mathbb{R}^{3}} e^{i \mathbf{p x}}\left[\psi_{t}\left(p_{+}(\mathbf{p})\right)-\psi_{t}\left(p_{-}(\mathbf{p})\right)\right] \frac{m^{2} d^{3} \mathbf{p}}{E(\mathbf{p})} \\
& =\frac{(2 \pi)^{-3 / 2}}{m} \int_{\mathbb{R}^{3}}\left[e^{-i p_{+}(\mathbf{p}) x} \psi\left(p_{+}(\mathbf{p})\right)-e^{-i p_{-}(\mathbf{p}) x} \psi\left(p_{-}(\mathbf{p})\right)\right] \frac{m^{2} d^{3} \mathbf{p}}{E(\mathbf{p})} \\
& =\frac{(2 \pi)^{-3 / 2}}{m} \int_{\mathcal{M}} e^{-i p x} \psi(p) i_{p}\left(d^{4} p\right) \quad \text { by }(28), \\
& =\mathcal{F}_{0 \mathcal{M}} \psi(x) .
\end{aligned}
$$

This shows that $\mathcal{F}_{0 \mathcal{M}} \psi$ is supported in $\{0\} \times \overline{B_{\alpha}^{3}(0)}+$ Causal. Consequently, $\mathcal{F}_{0 \mathcal{M}}$ maps $\mathcal{C}_{\mathcal{M}}$ to $\mathcal{C}_{0}$.

Finally, We CONSIDER $\mathcal{F}_{\Sigma 0}$. Let $\psi \in \mathcal{C}_{0}$ be supported in $K+$ Causal with some compact set $K \subset \mathbb{R}^{4}$. Because $\psi$ is smooth, its restriction to $\Sigma$ is also smooth. Moreover, $(K+$ Causal $) \cap \Sigma$ is compact. This shows that $\mathcal{F}_{\Sigma 0} \psi \in \mathcal{C}_{\Sigma}$.

Lemma 3.6. For $\psi \in \mathcal{C}_{\Sigma}$, the function $\mathcal{F}_{0 \mathcal{M}} \mathcal{F}_{\mathcal{M} \Sigma} \psi$ is supported in $\operatorname{supp} \psi+$ Causal. Furthermore, the following identities hold:

$$
\begin{aligned}
& \mathcal{F}_{\Sigma 0} \mathcal{F}_{0 \mathcal{M}} \mathcal{F}_{\mathcal{M} \Sigma}=\operatorname{id}_{\mathcal{C}_{\Sigma}}, \\
& \mathcal{F}_{\mathcal{M} \Sigma} \mathcal{F}_{\Sigma 0} \mathcal{F}_{0 \mathcal{M}}=\operatorname{id}_{\mathcal{C}_{\mathcal{M}}}, \\
& \mathcal{F}_{0 \mathcal{M}} \mathcal{F}_{\mathcal{M} \Sigma} \mathcal{F}_{\Sigma 0}=\operatorname{id}_{\mathcal{C}_{0}} .
\end{aligned}
$$

Finally, the maps $\mathcal{F}_{\mathcal{M} \Sigma}: \mathcal{C}_{\Sigma} \rightarrow \mathcal{C}_{\mathcal{M}}, \mathcal{F}_{\Sigma 0}: \mathcal{C}_{0} \rightarrow \mathcal{C}_{\Sigma}$, and $\mathcal{F}_{0 \mathcal{M}}: \mathcal{C}_{\mathcal{M}} \rightarrow \mathcal{C}_{0}$ are isometric isomorphisms.

Proof. We abbreviate

$$
\mathcal{F}_{0 \Sigma}:=\mathcal{F}_{0 \mathcal{M}} \mathcal{F}_{\mathcal{M} \Sigma}
$$

We EXAmine First the SUPport of $\mathcal{F}_{0 \Sigma}$ AND of $\mathcal{F}_{\Sigma 0} \mathcal{F}_{0 \Sigma} \psi$ FOR $\psi \in \mathcal{C}_{\Sigma}$. We claim: If the support of $\psi$ is contained in $\overline{B_{r}^{4}(y)}$ for some $y \in \Sigma$, then the support of $\mathcal{F}_{0 \Sigma} \psi$ is contained in $y+\{0\} \times \overline{B_{\sqrt{2} r}^{3}(0)}+$ Causal. We prove this first for the special case $y=0 \in \Sigma$. Under the above assumptions, Lemma 3.4 yields $\left\|\mathcal{F}_{\mathcal{M} \Sigma} \psi\right\|_{\mathcal{M}, \sqrt{2} \alpha, n}<\infty$ for all $\alpha>r$ and all $n \in \mathbb{N}$. Using Lemma 3.5, it follows that the support of $\mathcal{F}_{0 \Sigma}(\psi)$ is contained in $\{0\} \times \overline{B_{\sqrt{2} \alpha}^{3}(y)}+$ Causal. 
Because $\alpha>r$ is arbitrary, this implies that $\operatorname{supp}\left(\mathcal{F}_{0 \Sigma} \psi\right) \subseteq\{0\} \times \overline{B_{\sqrt{2} r}^{3}(y)}+$ Causal. Next, we reduce the general case $y \in \Sigma$ to the special case $y=0$, using the translation maps $T_{\Sigma}^{-y}: \mathcal{C}_{\Sigma} \rightarrow \mathcal{C}_{\Sigma-y}$ and $T_{0}^{-y}: \mathcal{C}_{0} \rightarrow \mathcal{C}_{0}$ from Definition 2.10. By equations (64) and (65) in Lemma 2.15, these maps fulfill

$$
T_{0}^{-y} \mathcal{F}_{0 \Sigma}=\mathcal{F}_{0, \Sigma-y} T_{\Sigma}^{-y}
$$

Given that $\psi$ is supported in a subset of $\overline{B_{r}^{4}(y)}$, it follows that $T_{\Sigma}^{-y} \psi$ is supported in a subset of $\overline{B_{r}^{4}(0)}$. Using the special case from above, it follows that $T_{0}^{-y} \mathcal{F}_{0 \Sigma} \psi=\mathcal{F}_{0, \Sigma-y} T_{\Sigma}^{-y} \psi$ is supported in $\{0\} \times \overline{B_{\sqrt{2} r}^{3}(0)}+$ Causal. But then $\mathcal{F}_{0 \Sigma} \psi$ is supported in $y+\{0\} \times \overline{B_{\sqrt{2} r}^{3}(0)}+$ Causal.

We PROVE NOW THE FIRST CLAIM OF THE LEMMA. Let $r>0$. Using a compactness argument and a partition of unity, we can take finitely many points $y_{1}, \ldots, y_{k} \in \operatorname{supp} \psi$ and $\psi_{1}, \ldots, \psi_{k} \in \mathcal{C}_{\Sigma}$ with $\sum_{j=1}^{k} \psi_{j}=\psi$, such that for $j=1, \ldots, k$, we have $\operatorname{supp} \psi_{j} \subseteq B_{r}^{4}\left(y_{j}\right)$. But then

$$
\operatorname{supp}\left(\mathcal{F}_{0 \Sigma} \psi_{j}\right) \subseteq y_{j}+\{0\} \times \overline{B_{\sqrt{2} r}^{3}(0)}+\text { Causal, } \quad j=1, \ldots, k
$$

We conclude

$$
\operatorname{supp}\left(\mathcal{F}_{0 \Sigma} \psi\right) \subseteq \bigcup_{j=1}^{k}\left(y_{j}+\{0\} \times \overline{B_{\sqrt{2} r}^{3}(0)}+\text { Causal }\right) \subseteq \operatorname{supp} \psi+\{0\} \times \overline{B_{\sqrt{2} r}^{3}(0)}+\text { Causal }
$$

Because $r>0$ is arbitrary, this proves the claim

$$
\operatorname{supp}\left(\mathcal{F}_{0 \Sigma} \psi\right) \subseteq \bigcap_{r>0}\left(\operatorname{supp} \psi+\{0\} \times \overline{B_{\sqrt{2} r}^{3}(0)}+\text { Causal }\right)=\operatorname{supp} \psi+\text { Causal }
$$

We get for any $\psi \in \mathcal{C}_{\Sigma}$, using that $\operatorname{supp} \psi \subseteq \Sigma$ is space-like:

$$
\operatorname{supp}\left(\mathcal{F}_{\Sigma 0} \mathcal{F}_{0 \Sigma} \psi\right) \subseteq \Sigma \cap(\operatorname{supp} \psi+\text { Causal })=\operatorname{supp} \psi \text {. }
$$

Next, We Prove Equation (149): Given $\psi \in \mathcal{C}_{\Sigma}$ and $y \in \Sigma$, we need to show

$$
\mathcal{F}_{\Sigma 0} \mathcal{F}_{0 \Sigma} \psi(y)=\psi(y) \text {. }
$$

We prove this first in the special case that $y=0 \in \Sigma$ and that the tangent space of $\Sigma$ in 0 equals $T_{0} \Sigma=\{0\} \times \mathbb{R}^{3}$, and then reduce the general case to the special case, using a translation and a Lorentz transformation.

Let $\mathbb{R}^{3} \ni \mathbf{x} \mapsto\left(t_{\Sigma}(\mathbf{x}), \mathbf{x}\right) \in \Sigma$ be the representation of $\Sigma$ as a graph as in (7). In particular our assumption means $t_{\Sigma}(0)=0$ and $\nabla t_{\Sigma}(0)=0$. We set $\eta=\sup _{\mathbf{x} \in \mathbb{R}^{3}}\left|\nabla t_{\Sigma}(\mathbf{x})\right|<1$; recall condition (c) in the definition of Cauchy surfaces (Def. 2.2). Then for every $x=\left(x^{0}, \mathbf{x}\right) \in$ $\Sigma \backslash\{0\}$ and every $y=(0, \mathbf{y}) \in\{0\} \times \mathbb{R}^{3}$ with $|\mathbf{y}|<(1-\eta)|\mathbf{x}|$, the vector $x-y$ is space-like. Indeed, $\left|x^{0}-y^{0}\right|=\left|x^{0}\right| \leqslant \eta|\mathbf{x}|<|\mathbf{x}|-|\mathbf{y}| \leqslant|\mathbf{x}-\mathbf{y}|$. Let $\phi: \mathbb{R}^{3} \rightarrow \mathbb{R}_{0}^{+}$be a smooth function supported in the open ball $B_{1-\eta}^{3}(0)$ with

$$
\int_{\mathbb{R}^{3}} \phi(\mathbf{x}) d^{3} \mathbf{x}=1
$$


and let $\chi: \mathbb{R}^{3} \rightarrow[0,1]$ be another smooth, compactly supported function which equals 1 in $\overline{B_{1}^{3}(0)}$. For every $\epsilon>0$, we introduce $\chi_{\epsilon}: \Sigma \rightarrow[0,1], \chi_{\epsilon}\left(x^{0}, \mathbf{x}\right)=\chi(\mathbf{x} / \epsilon)$ and $\phi_{\epsilon}: \mathbb{R}^{3} \rightarrow \mathbb{R}_{0}^{+}$, $\phi_{\epsilon}(\mathbf{x})=\epsilon^{-3} \phi(\mathbf{x} / \epsilon)$. Note that $\phi_{\epsilon}$ fulfills

$$
\int_{\mathbb{R}^{3}} \phi_{\epsilon}(\mathbf{x}) d^{3} \mathbf{x}=1 .
$$

Furthermore, for every $x=\left(x^{0}, \mathbf{x}\right) \in \operatorname{supp}\left(\left(1-\chi_{\epsilon}\right) \psi\right)$, we have $|\mathbf{x}| \geqslant \epsilon$, and every $y=$ $(0, \mathbf{y}) \in\{0\} \times \operatorname{supp} \phi_{\epsilon}$ fulfills $|\mathbf{y}|<(1-\eta) \epsilon$. Hence $x-y$ is space-like. It follows that the sets $\operatorname{supp}\left(\left(1-\chi_{\epsilon}\right) \psi\right)+$ Causal and $\{0\} \times \operatorname{supp} \phi_{\epsilon}$ are disjoint. Using

$$
\operatorname{supp}\left(\mathcal{F}_{0 \mathcal{M}} \mathcal{F}_{\mathcal{M} \Sigma}\left(\left(1-\chi_{\epsilon}\right) \psi\right) \subseteq \operatorname{supp}\left(\left(1-\chi_{\epsilon}\right) \psi\right)+\right.\text { Causal }
$$

we conclude for all $\mathbf{x} \in \mathbb{R}^{3}, x=(0, \mathbf{x})$ and $\epsilon>0$ that $\phi_{\epsilon}(\mathbf{x})=0$ or $\mathcal{F}_{0 \Sigma}\left(\left(1-\chi_{\epsilon}\right) \psi\right)(x)=0$ holds, i.e.,

$$
\phi_{\epsilon}(\mathbf{x}) \mathcal{F}_{0 \Sigma} \psi(x)=\phi_{\epsilon}(\mathbf{x}) \mathcal{F}_{0 \Sigma}\left(\chi_{\epsilon} \psi\right)(x) .
$$

Integrating the left hand side over $\mathbf{x}$ and taking the limit as $\epsilon \downarrow 0$ we get on the one hand, using continuity of the function $\mathcal{F}_{0 \Sigma} \psi$ :

$$
\lim _{\epsilon \downarrow 0} \int_{\mathbb{R}^{3}} \phi_{\epsilon}(\mathbf{x}) \cdot \mathcal{F}_{0 \Sigma} \psi(0, \mathbf{x}) d^{3} \mathbf{x}=\mathcal{F}_{0 \Sigma} \psi(0) .
$$

On the other hand, we integrate also the right hand side of (162) and rewrite it as

$$
\begin{aligned}
& \int_{\mathbb{R}^{3}} \phi_{\epsilon}(\mathbf{x})\left[\mathcal{F}_{0 \Sigma}\left(\chi_{\epsilon} \psi\right)\right](0, \mathbf{x}) d^{3} \mathbf{x} \\
& =\frac{(2 \pi)^{-3 / 2}}{m} \epsilon^{-3} \int_{\mathbf{x} \in \mathbb{R}^{3}} \phi(\mathbf{x} / \epsilon) \int_{p=\left(p^{0}, \mathbf{p}\right) \in \mathcal{M}} e^{i \mathbf{p x}} \mathcal{F}_{\mathcal{M} \Sigma}\left(\chi_{\epsilon} \psi\right)(p) i_{p}\left(d^{4} p\right) d^{3} \mathbf{x} \\
& =\frac{(2 \pi)^{-3 / 2}}{m} \epsilon^{-3} \int_{\mathcal{M}} \int_{\mathbb{R}^{3}} \phi(\mathbf{x} / \epsilon) e^{i \mathbf{p x}} d^{3} \mathbf{x} \mathcal{F}_{\mathcal{M} \Sigma}\left(\chi_{\epsilon} \psi\right)(p) i_{p}\left(d^{4} p\right) \\
& =m^{-1} \int_{\mathcal{M}} \hat{\phi}(\epsilon \mathbf{p}) \mathcal{F}_{\mathcal{M} \Sigma}\left(\chi_{\epsilon} \psi\right)(p) i_{p}\left(d^{4} p\right)
\end{aligned}
$$

with the Fourier integral being

$$
\hat{\phi}(\mathbf{q}):=(2 \pi)^{-3 / 2} \int_{\mathbb{R}^{3}} \phi(\mathbf{x}) e^{i \mathbf{q x}} d^{3} \mathbf{x}, \quad \mathbf{q} \in \mathbb{R}^{3} .
$$

Note that changing the order of integration in (164) is justified because $\phi$ is compactly supported and because $\mathcal{F}_{\mathcal{M} \Sigma}\left(\chi_{\epsilon} \psi\right)(p)$ decays faster than any power of $|p|$ as $|p| \rightarrow \infty, p \in \mathcal{M}$; 
here we use that $\mathcal{F}_{\mathcal{M} \Sigma}\left(\chi_{\epsilon} \psi\right) \in \mathcal{C}_{\mathcal{M}}$ by Lemma 3.4. Using the definition (61) of $\mathcal{F}_{\mathcal{M} \Sigma}$, formulas (23)-(24) and (28), and the representation (14) of $i_{\gamma}\left(d^{4} x\right)$, the quantity in (164) equals

$$
\begin{aligned}
& \frac{(2 \pi)^{-3 / 2}}{m} \int_{p \in \mathcal{M}} \hat{\phi}(\epsilon \mathbf{p}) \frac{\not p+m}{2 m} \int_{x \in \Sigma} e^{i p x} i_{\gamma}\left(d^{4} x\right) \chi_{\epsilon}(x) \psi(x) i_{p}\left(d^{4} p\right) \\
= & (2 \pi)^{-3 / 2} \int_{p \in \mathcal{M}} \hat{\phi}(\epsilon \mathbf{p}) P(p) \int_{x \in \Sigma} e^{i p x} \gamma^{0} i_{\gamma}\left(d^{4} x\right) \chi_{\epsilon}(x) \psi(x) d^{3} p \\
= & (2 \pi)^{-3 / 2} \int_{\mathbf{p} \in \mathbb{R}^{3}} \hat{\phi}(\epsilon \mathbf{p}) \int_{\mathbf{x} \in \mathbb{R}^{3}}\left(P_{+}(\mathbf{p}) e^{i E(\mathbf{p}) t_{\Sigma}(\mathbf{x})}+P_{-}(\mathbf{p}) e^{-i E(\mathbf{p}) t_{\Sigma}(\mathbf{x})}\right) e^{-i \mathbf{p x}} \times \\
& \times\left(1+\sum_{\mu=1}^{3} \gamma^{0} \gamma^{\mu} \partial_{\mu} t_{\Sigma}(\mathbf{x})\right) \chi(\mathbf{x} / \epsilon) \psi\left(t_{\Sigma}(\mathbf{x}), \mathbf{x}\right) d^{3} \mathbf{x} d^{3} \mathbf{p} \\
= & (2 \pi)^{-3 / 2} \int_{\mathbf{q} \in \mathbb{R}^{3}} \hat{\phi}(\mathbf{q}) \int_{\mathbf{y} \in \mathbb{R}^{3}}\left(P_{+}(\mathbf{q} / \epsilon) e^{i E(\mathbf{q} / \epsilon) t_{\Sigma}(\epsilon \mathbf{y})}+P_{-}(\mathbf{q} / \epsilon) e^{-i E(\mathbf{q} / \epsilon) t_{\Sigma}(\epsilon \mathbf{y})}\right) e^{-i \mathbf{q} \mathbf{y}} \times \\
& \times\left(1+\sum_{\mu=1}^{3} \gamma^{0} \gamma^{\mu} \partial_{\mu} t_{\Sigma}(\epsilon \mathbf{y})\right) \chi(\mathbf{y}) \psi\left(t_{\Sigma}(\epsilon \mathbf{y}), \epsilon \mathbf{y}\right) d^{3} \mathbf{y} d^{3} \mathbf{q}
\end{aligned}
$$

We now take the limit as $\epsilon$ tends to zero using dominated convergence and exploit the following ingredients:

(a) To find a dominating function for the integrand we employ:

(i) $P_{+}$and $P_{-}$take values in the set of orthogonal projectors and therefore are bounded;

(ii) $\nabla t_{\Sigma}$ and $\psi$ are bounded;

(iii) $\hat{\phi}(\mathbf{q})$ is bounded and decays faster than any power of $|\mathbf{q}|$ for $|\mathbf{q}| \rightarrow \infty$;

(iv) $\chi$ is bounded and compactly supported;

(b) For the point-wise convergence as of the integrand $\epsilon \rightarrow 0$ we use for any $\mathbf{q}, \mathbf{x}$ :

(i) $P_{ \pm}(\mathbf{q} / \epsilon)$ converge to orthogonal projectors and $P_{+}(\mathbf{q} / \epsilon)+P_{-}(\mathbf{q} / \epsilon)$ equals the identity;

(ii) $t_{\Sigma}(\epsilon \mathbf{y}) / \epsilon \rightarrow 0$ and $E(\mathbf{q} / \epsilon) \epsilon$ is bounded for sufficiently small $\epsilon$;

(iii) $\nabla t_{\Sigma}(\epsilon \mathbf{y}) \rightarrow 0$;

(iv) $\psi\left(t_{\Sigma}(\epsilon \mathbf{y}), \epsilon \mathbf{y}\right) \rightarrow \psi(0)$.

This implies that the limit of (166) as $\epsilon \rightarrow 0$ can be expressed as

$$
\begin{aligned}
& \psi(0)(2 \pi)^{-3 / 2} \int_{\mathbf{q} \in \mathbb{R}^{3}} \hat{\phi}(\mathbf{q}) \int_{\mathbf{y} \in \mathbb{R}^{3}} e^{-i \mathbf{q y}} \chi(\mathbf{y}) d^{3} \mathbf{y} d^{3} \mathbf{q} \\
= & \psi(0) \int_{\mathbf{y} \in \mathbb{R}^{3}} \phi(\mathbf{y}) \chi(\mathbf{y}) d^{3} \mathbf{y}=\psi(0) \int_{\mathbf{y} \in \mathbb{R}^{3}} \phi(\mathbf{y}) d^{3} \mathbf{y} \\
= & \psi(0)
\end{aligned}
$$


because by the choice of $\phi$ and $\chi$ we have $\phi \chi=\phi$; recall also formula (159). Let us summarize. Together with (162), (163) we have shown that

$$
\mathcal{F}_{0 \Sigma} \psi(0)=\psi(0)
$$

which implies

$$
\mathcal{F}_{\Sigma 0} \mathcal{F}_{0 \Sigma} \psi(0)=\psi(0)
$$

Next, we treat the case of general $y \in \Sigma$ with a general tangent space $T_{y} \Sigma$, using a translation by $-y$ and a Lorentz transformation encoded by some $(S, \Lambda)$ that maps the space-like hyperplane $T_{0}(\Sigma-y)$ to the time-0-hyperplane $\{0\} \times \mathbb{R}^{3}$. Using Theorem 2.15 together with the special case just considered, we get

$$
\begin{aligned}
& \mathcal{F}_{\Sigma 0} \mathcal{F}_{0 \Sigma} \psi(y)=\mathcal{F}_{0 \Sigma} \psi(y)=T_{0}^{-y} \mathcal{F}_{0 \Sigma} \psi(0)=\mathcal{F}_{0, \Sigma-y} T_{\Sigma}^{-y} \psi(0) \\
& =\left(L_{0}^{(S, \Lambda)}\right)^{-1} \mathcal{F}_{0, \Lambda(\Sigma-y)} L_{\Sigma-y}^{(S, \Lambda)} T_{\Sigma}^{-y} \psi(0)=S^{-1} \cdot L_{\Sigma-y}^{(S, \Lambda)} T_{\Sigma}^{-y} \psi(0)=T_{\Sigma}^{-y} \psi(0)=\psi(y) .
\end{aligned}
$$

This proves equation (149).

By Definition (15) of the scalar product in $\mathcal{C}_{0}$, the map $\mathcal{F}_{\Sigma 0}: \mathcal{C}_{0} \rightarrow \mathcal{C}_{\Sigma}$ is an isometry. Using equation (149), i.e., $\mathcal{F}_{\Sigma 0} \mathcal{F}_{0 \Sigma}=\mathrm{id}_{\mathcal{C}_{\Sigma}}$, it follows that $\mathcal{F}_{0 \Sigma}$ is also an isometry.

Now We PROVE THAT $\mathcal{F}_{0 \mathcal{M}}: \mathcal{C}_{\mathcal{M}} \rightarrow \mathcal{C}_{0}$ IS AN ISOMETRY. We consider the time-zerohyperplane $\Sigma^{0}=\{0\} \times \mathbb{R}^{3}$ and set $\mathcal{F}_{\Sigma^{0} 3}:=\mathcal{F}_{\Sigma^{0} 0} \mathcal{F}_{0 \mathcal{M}} \mathcal{F}_{\mathcal{M} 3}$.It is just the standard inverse Fourier transform, as the following calculation shows. For $\psi \in \mathcal{C}_{3}$ and $\mathbf{x} \in \mathbb{R}^{3}$, combining (63), (62), and (60) with (23), (25), (24), and (28), we obtain

$$
\left(\mathcal{F}_{\Sigma^{0} 3} \psi\right)(0, \mathbf{x})=(2 \pi)^{-3 / 2} \int_{\mathbb{R}^{3}} e^{i \mathbf{p x}}\left(P_{+}(\mathbf{p})+P_{-}(\mathbf{p})\right) \psi(\mathbf{p}) d^{3} \mathbf{p}=(2 \pi)^{-3 / 2} \int_{\mathbb{R}^{3}} e^{i \mathbf{p x}} \psi(\mathbf{p}) d^{3} \mathbf{p}
$$

As a consequence, $\mathcal{F}_{\Sigma^{0} 3} \psi: \mathcal{C}_{3} \rightarrow \mathcal{C}_{\Sigma^{0}}$ is isometric. Since $\mathcal{F}_{\Sigma^{0} 0}$ and $\mathcal{F}_{\mathcal{M} 3}$ are isometries and $\mathcal{F}_{\mathcal{M} 3}\left[\mathcal{C}_{3}\right]=\mathcal{C}_{\mathcal{M}}$ by Lemma 3.1, it follows that $\mathcal{F}_{0 \mathcal{M}}: \mathcal{C}_{\mathcal{M}} \rightarrow \mathcal{C}_{0}$ is also an isometry.

As $\mathcal{F}_{\Sigma 0}: \mathcal{C}_{0} \rightarrow \mathcal{C}_{\Sigma}$ and $\mathcal{F}_{0 \mathcal{M}}: \mathcal{C}_{\mathcal{M}} \rightarrow \mathcal{C}_{0}$ are isometric, formula (149) implies that $\mathcal{F}_{\mathcal{M} \Sigma}: \mathcal{C}_{\Sigma} \rightarrow \mathcal{C}_{\mathcal{M}}$ is also isometric.

Finally, We PROve Equations (150) And (151). We use the following well-known fact. Assume that isometries $f: C \rightarrow C^{\prime}$ and $g: C^{\prime} \rightarrow C$ between pre-Hilbert spaces $C, C^{\prime}$ are given. Further assume that $g \circ f=\operatorname{id}_{C}$ holds. Then $f$ and $g$ are isometric isomorphisms and inverse to each other. We apply this fact to $g=\mathcal{F}_{\Sigma 0} \mathcal{F}_{0 \mathcal{M}}$ and $f=\mathcal{F}_{\mathcal{M} \Sigma}$ on the one hand to get equation (150) from equation (149), and to $g=\mathcal{F}_{\Sigma 0}$ and $f=\mathcal{F}_{0 \mathcal{M}} \mathcal{F}_{\mathcal{M} \Sigma}$ on the other hand to get equation (151) also from equation (149). The three equations (149)-(151) show also that the three maps $\mathcal{F}_{\mathcal{M} \Sigma}: \mathcal{C}_{\Sigma} \rightarrow \mathcal{C}_{\mathcal{M}}, \mathcal{F}_{\Sigma 0}: \mathcal{C}_{0} \rightarrow \mathcal{C}_{\Sigma}$, and $\mathcal{F}_{0 \mathcal{M}}: \mathcal{C}_{\mathcal{M}} \rightarrow \mathcal{C}_{0}$ are isomorphisms.

As a consequence we get the following corollary. 
Corollary 3.7. The maps $\mathcal{F}_{\mathcal{M} \Sigma}: \mathcal{C}_{\Sigma} \rightarrow \mathcal{C}_{\mathcal{M}}, \mathcal{F}_{\Sigma 0}: \mathcal{C}_{0} \rightarrow \mathcal{C}_{\Sigma}$, and $\mathcal{F}_{0 \mathcal{M}}: \mathcal{C}_{\mathcal{M}} \rightarrow \mathcal{C}_{0}$ extend to unitary maps

$$
\mathcal{F}_{\mathcal{M} \Sigma}: \mathcal{H}_{\Sigma} \rightarrow \mathcal{H}_{\mathcal{M}}, \quad \mathcal{F}_{\Sigma 0}: \mathcal{H}_{0} \rightarrow \mathcal{H}_{\Sigma}, \quad \mathcal{F}_{0 \mathcal{M}}: \mathcal{H}_{\mathcal{M}} \rightarrow \mathcal{H}_{0}
$$

Furthermore, they fulfill

$$
\begin{aligned}
& \mathcal{F}_{\Sigma 0} \mathcal{F}_{0 \mathcal{M}} \mathcal{F}_{\mathcal{M} \Sigma}=\operatorname{id}_{\mathcal{H}_{\Sigma}} \\
& \mathcal{F}_{\mathcal{M} \Sigma} \mathcal{F}_{\Sigma 0} \mathcal{F}_{0 \mathcal{M}}=\operatorname{id}_{\mathcal{H}_{\mathcal{M}}}, \\
& \mathcal{F}_{0 \mathcal{M}} \mathcal{F}_{\mathcal{M} \Sigma} \mathcal{F}_{\Sigma 0}=\operatorname{id}_{\mathcal{H}_{0}}
\end{aligned}
$$

Proof. This follows immediately from Lemma 3.6, because $\mathcal{C}_{\Sigma}, \mathcal{C}_{0}$, and $\mathcal{C}_{\mathcal{M}}$ are dense in $\mathcal{H}_{\Sigma}$, $\mathcal{H}_{0}$, and $\mathcal{H}_{\mathcal{M}}$, respectively.

\subsubsection{Proof of Theorem 2.14}

Proof of Theorem 2.14. (a) For any placeholders $I, J, K$ among the symbols $3, \mathcal{M}, 0$ or any Cauchy surface $\Sigma$ such that $\mathcal{F}_{I J}$ and $\mathcal{F}_{J K}$ are already defined, but $\mathcal{F}_{I K}$ is not yet defined, we define $\mathcal{F}_{I K}:=\mathcal{F}_{I J} \mathcal{F}_{J K}$. This is repeated recursively until all maps $\mathcal{F}_{I K}$ are defined. As a consequence of Lemmas 3.1 and 3.6 and Corollary 3.7, these recursive definitions do not contradict each other. All claims of Theorem 2.14 (a) follow now immediately. Claim (b) is already proven in Lemma 3.6, and claim (c) is just composed of Lemma 2.9, Corollary 3.3, Definition 2.4, and equation (16).

\subsection{Existence, Uniqueness, and Causal Structure}

In this section the Theorems $2.20,2.22$, and 2.23 are proven. The strategy of proof is the following:

(1) Proof of existence and uniqueness of solutions in the interaction picture introduced in Section 2.7; see Lemma 3.9.

(2) Proof of regularity and support properties of solutions in the interaction picture; see Theorem 2.23.

(3) Use (2) to prove the equivalence of the Schrödinger and the interaction picture in Section 3.2.2.

(4) Use (1), (2), and (3) to prove existence, uniqueness, regularity, and causal structure of solutions for the Dirac equation in Sections 3.2.4 and 3.2.5.

\subsubsection{Existence and Uniqueness in the Interaction Picture}

Preliminarily we check general properties of the operator $L_{t}$ defined in (85). 
Lemma 3.8. For any $t \in \mathbb{R}$ and $n \in \mathbb{N}_{0}$, the operator $L_{t}: \mathcal{C}_{0} \supset$ introduced in (85) extends to a bounded linear map $L_{t}:\left(\mathcal{H}_{0, n},\|\cdot\|_{0, n}\right) \subseteq$, denoted by the same symbol $L_{t}$. For any $n, l \in \mathbb{N}_{0}$ with $n \geqslant l$ the function

$$
L_{(\cdot)}: \mathbb{R} \rightarrow \mathcal{B}\left(\mathcal{H}_{0, n},\|\cdot\|_{0, n}\right) \subseteq \mathcal{B}\left(\left(\mathcal{H}_{0, n},\|\cdot\|_{0, n}\right),\left(\mathcal{H}_{0, n-l},\|\cdot\|_{0, n-l}\right)\right), \quad t \mapsto L_{t}
$$

is $l$ times continuously differentiable with respect to the strong operator topology on $\mathcal{B}\left(\left(\mathcal{H}_{0, n},\|\cdot\|_{0, n}\right),\left(\mathcal{H}_{0, n-l},\|\cdot\|_{0, n-l}\right)\right)$, where "0 times continuously differentiable" means "continuous". In particular, for all $n \in \mathbb{N}_{0}$ the operator norm of $L_{t}$ is locally bounded in $t \in \mathbb{R}$, i.e., $\sup _{t \in\left[t_{0}, t_{1}\right]}\left\|L_{t}\right\|_{\mathcal{H}_{0, n} \rightarrow \mathcal{H}_{0, n}}<\infty$ for all reals $t_{0}<t_{1}$.

Proof. Let $t \in \mathbb{R}$ and $n \in \mathbb{N}_{0}$ (not to be confused with the normal vector field $n=n(x)$ having the same name). By applying Lemma A.2 from the appendix to the function $Z(\mathbf{x})=\left(v_{t} h_{t} A\right)\left(t_{\Sigma_{t}}(\mathbf{x}), \mathbf{x}\right), \mathbf{x} \in \mathbb{R}^{3}$, it follows that the multiplication operator $v_{t} h_{t} A$ : $\left(\mathcal{H}_{\Sigma_{t}, n},\|\cdot\|_{\Sigma_{t}, n}\right) \supset$ is bounded. Consequently, $L_{t}=\mathcal{F}_{0 \Sigma_{t}}\left(v_{t} \eta_{t} \mathcal{A}\right) \mathcal{F}_{\Sigma_{t} 0}:\left(\mathcal{H}_{0, n},\|\cdot\|_{0, n}\right) \supset$ is bounded as well.

Let $n, l \in \mathbb{N}_{0}$ such that $n \geqslant l$. It suffices to check that the maps $t \mapsto L_{t} \psi \in \mathcal{H}_{0, n-l}$ have the regularity $\mathcal{C}^{l}$ w.r.t. the norm $\|\cdot\|_{0, n-l}$ for all $\psi$ in the dense subset $\mathcal{C}_{0}$ of $\mathcal{H}_{0, n}$. Furthermore, it even suffices to check the $\mathcal{C}^{l}$ regularity of

$$
t \mapsto \mathcal{F}_{\mathcal{M} 0} L_{t} \psi=\mathcal{F}_{\mathcal{M} \Sigma_{t}}\left(v_{t} h_{t} A\right) \mathcal{F}_{\Sigma_{t}, 0} \psi \in\left(\mathcal{H}_{\mathcal{M}, n-l},\|\cdot\|_{\mathcal{M}, n-l}\right) .
$$

Using Theorem 2.14 we find for $p \in \mathcal{M}$

$$
\mathcal{F}_{\mathcal{M} 0} L_{t} \psi(p)=\frac{\not p+m}{2 m}(2 \pi)^{-3 / 2} \int_{\Sigma_{t}} e^{i p x} i_{\gamma}\left(d^{4} x\right) v_{t}(x) h_{t}(x) \mathcal{A}(x) \psi(x)
$$

We recall that the motion of $\Sigma_{t}$ can be seen as driven by the vector field $v(x) n(x)$, that $A$ is compactly supported, and that $v(x), n(x), A(x), \psi(x)$ are smooth. Therefore, the following derivatives w.r.t. $t$, point-wise in $p \in \mathcal{M}$, exist and are given by

$$
\frac{\partial^{l}}{\partial t^{l}} \mathcal{F}_{\mathcal{M} 0} L_{t} \psi(p)=\frac{\not p+m}{2 m}(2 \pi)^{-3 / 2} \int_{\Sigma_{t}} \mathcal{L}_{v_{t} n_{t}}^{l}\left(e^{i p x} i_{\gamma}\left(d^{4} x\right) v_{t}(x) h_{t}(x) A(x) \psi(x)\right),
$$

where $\mathcal{L}_{v_{t} n_{t}}=i_{v_{t} n_{t}} \circ d+d \circ i_{v_{t} n_{t}}$ denotes the Lie derivative. Expanding the iterated Lie derivative, the integrand takes the form

$$
\mathcal{L}_{v_{t} n_{t}}^{l}\left(e^{i p x} i_{\gamma}\left(d^{4} x\right) v_{t}(x) h_{t}(x) \mathcal{A}(x) \psi(x)\right)=\sum_{\substack{\alpha, \beta \in \mathbb{N}_{0}^{4} \\|\alpha+\beta| \leqslant l}} p^{\beta} e^{i p x} i_{\gamma}\left(d^{4} x\right) \zeta_{l, \alpha, \beta}(x) \partial^{\alpha} \psi(x)
$$

for appropriate $\zeta_{l, \alpha, \beta} \in \mathcal{C}_{c}^{\infty}\left(\mathbb{R}^{4}, \mathbb{C}^{4 \times 4}\right)$ being independent of $p$ and $\psi$. It follows

$$
\frac{\partial^{l}}{\partial t^{l}} \mathcal{F}_{\mathcal{M} 0} L_{t} \psi(p)=\left.\sum_{\substack{\alpha, \beta \in \mathbb{N}_{0}^{4} \\|\alpha+\beta| \leqslant l}} p^{\beta} \mathcal{F}_{\mathcal{M} \Sigma_{t}}\left(\zeta_{l, \alpha, \beta} \partial^{\alpha} \psi\right)\right|_{\Sigma_{t}}(p), \quad \text { for } p \in \mathcal{M}
$$


Using Lemma 3.4 we observe for our given $\psi \in \mathcal{C}_{0}$ that for all bounded intervals $\left[t_{0}, t_{1}\right]$ there exists $\gamma>0$ such that for all $k \in \mathbb{N}$

$$
\sup _{\substack{t \in\left[t_{0}, t_{1}\right] \\ \alpha, \beta \in \mathbb{N}_{0}^{4},|\alpha+\beta| \leqslant l}}\left\|\left.\mathcal{F}_{\mathcal{M} \Sigma_{t}}\left(\zeta_{l, \alpha, \beta} \partial^{\alpha} \psi\right)\right|_{\Sigma_{t}}\right\|_{\mathcal{M}, \gamma, k}<\infty
$$

holds. Using dominated convergence we infer that

$$
\left.\left[t_{0}, t_{1}\right] \ni t \mapsto \sum_{\substack{\alpha, \beta \in \mathbb{N}_{0}^{4} \\|\alpha+\beta| \leqslant l}} p^{\beta} \mathcal{F}_{\mathcal{M} \Sigma_{t}}\left(\zeta_{l, \alpha, \beta} \partial^{\alpha} \psi\right)\right|_{\Sigma_{t}} \in\left(\mathcal{H}_{\mathcal{M}, j},\|\cdot\|_{\mathcal{M}, j}\right)
$$

is continuous for all $j \in \mathbb{N}_{0}$ and equals $t \mapsto \frac{\partial^{l}}{\partial t} \mathcal{F}_{\mathcal{M} 0} L_{t} \psi$, where the derivatives are taken in $\left(\mathcal{H}_{\mathcal{M}, j},\|\cdot\|_{\mathcal{M}, j}\right)$. By Lemma A.2, Definition 2.18, and the fact that $\mathcal{C}_{0}$ is dense in $\mathcal{H}_{0, n}$, we conclude that the maps

$$
\left.\left(\mathcal{H}_{0, n},\|\cdot\|_{0, n}\right) \ni \phi \mapsto \sum_{\substack{\alpha, \beta \in \mathbb{N}_{0}^{4} \\|\alpha+\beta| \leqslant l}} p^{\beta} \mathcal{F}_{\mathcal{M} \Sigma_{t}}\left(\zeta_{l, \alpha, \beta} \partial^{\alpha} \phi\right)\right|_{\Sigma_{t}} \in\left(\mathcal{H}_{\mathcal{M}, n-l},\|\cdot\|_{\mathcal{M}, n-l}\right)
$$

are bounded uniformly in $t \in\left[t_{0}, t_{1}\right]$. Using this, the continuity of the map in (184), and again the denseness argument, we note that the continuity claimed in (184) holds also for any $\psi \in \mathcal{H}_{0, n}$ and $j=n-l$. By induction in $l=0,1, \ldots, n$ we find that for any $\phi \in \mathcal{H}_{0, n}$

$$
\frac{\partial^{l}}{\partial t^{l}} \mathcal{F}_{\mathcal{M} 0} L_{t} \phi=\left.\sum_{\substack{\alpha, \beta \in \mathbb{N}_{0}^{4} \\|\alpha+\beta| \leqslant l}} p^{\beta} \mathcal{F}_{\mathcal{M} \Sigma_{t}}\left(\zeta_{l, \alpha, \beta} \partial^{\alpha} \phi\right)\right|_{\Sigma_{t}}
$$

where the derivative in the induction step is taken in $\left(\mathcal{H}_{\mathcal{M}, n-l},\|\cdot\|_{\mathcal{M}, n-l}\right)$.

Lemma 3.9 (Existence and Uniqueness in the Interaction Picture). Let $n \in \mathbb{N}_{0}$. For any $\chi \in \mathcal{H}_{0, n}$, the initial value problem over $\left(\mathcal{H}_{0, n},\|\cdot\|_{0, n}\right)$

$$
i \frac{d}{d t} \phi_{t}=L_{t} \phi_{t}, \quad \phi_{0}=\chi
$$

has a unique solution $\phi_{(\cdot)}: \mathbb{R} \rightarrow \mathcal{H}_{0, n}$ which is continuously differentiable w.r.t. the norm $\|\cdot\|_{0, n}$.

Note that any solution of the initial value problem $(187)$ over $\left(\mathcal{H}_{0, n},\|\cdot\|_{0, n}\right)$ is also a solution over $\left(\mathcal{H}_{0, n^{\prime}},\|\cdot\|_{0, n^{\prime}}\right)$ for any $n^{\prime} \in \mathbb{N}_{0}$ with $n^{\prime} \leqslant n$. In particular, for initial data $\chi \in \bigcap_{n \in \mathbb{N}_{0}} \mathcal{H}_{0, n}$ the corresponding solution $\phi_{t}$ lies in the same intersection of spaces.

Proof. Lemma 3.8 ensures that the map $\mathbb{R} \ni t \mapsto L_{t} \in \mathcal{B}\left(\mathcal{H}_{0, n},\|\cdot\|_{0, n}\right)$ is continuous with respect to the strong operator topology. Consequently, by the Picard-Lindelöf theorem, it follows that the Volterra integral equation associated to (187)

$$
\phi_{t}=\chi-i \int_{0}^{t} L_{s} \phi_{s} d s
$$

has a unique continuous solution $\phi_{(\cdot)}: \mathbb{R} \rightarrow\left(\mathcal{H}_{0, n},\|\cdot\|_{0, n}\right)$ for any initial value $\chi \in \mathcal{H}_{0, n}$ and any $n \in \mathbb{N}_{0}$. Furthermore, the fundamental theorem of calculus guarantees that it is continuously differentiable with respect to the norm $\|\cdot\|_{0, n}$ with the derivative given in (187). 


\subsubsection{Regularity and Support Properties}

Lemma 3.10 (Regularity of Solutions). Let $n, l \in \mathbb{N}_{0}$ such that $n \geqslant l$.

(a) For any initial value $\chi \in \mathcal{H}_{0, n}$, the solution $\mathbb{R} \ni t \mapsto \phi_{t}$ of the initial value problem (187) is l times continuously differentiable w.r.t. the norm $\|\cdot\|_{0, n-l}$.

(b) If in addition $n \geqslant l+2$, then the map

$$
\phi: \mathbb{R}^{4} \times \mathbb{R} \rightarrow \mathbb{C}^{4}, \quad \phi(x, t)=\phi_{t}(x)
$$

is well-defined and $l$ times continuously differentiable. In particular, the function $\phi$ is smooth for initial values $\chi \in \mathcal{C}_{0}$ and solves the initial value problem (84), (85) in the classical sense.

In the proof of Lemma 3.10 we rely on the following lemma, which we prove first.

Lemma 3.11 (Derivatives of Translation Maps and Pointwise Evaluation).

(a) For any $n, l \in \mathbb{N}_{0}$ such that $n \geqslant l$, the family of translation maps

$$
T_{0}^{-\cdot}: \mathbb{R}^{4} \rightarrow \mathcal{B}\left(\left(\mathcal{H}_{0, n},\|\cdot\|_{0, n}\right),\left(\mathcal{H}_{0, n-l},\|\cdot\|_{0, n-l}\right)\right), \quad y \mapsto T_{0}^{-y}
$$

is l times continuously differentiable w.r.t. the strong operator topology with the derivatives

$$
\partial_{y}^{\alpha} T_{0}^{-y}=T_{0}^{-y} \partial^{\alpha} \in \mathcal{B}\left(\left(\mathcal{H}_{0, n},\|\cdot\|_{0, n}\right),\left(\mathcal{H}_{0, n-l},\|\cdot\|_{0, n-l}\right)\right), \quad\left(\partial_{y}^{\alpha} T_{0}^{-y}\right) \psi=\partial^{\alpha}\left(T_{0}^{-y} \psi\right)
$$

for every multi-index $\alpha \in \mathbb{N}_{0}^{4}$ with $|\alpha| \leqslant l$ and $\psi \in \mathcal{H}_{0, n}$.

(b) Given $k, n \in \mathbb{N}$ with $n \geqslant k+2$, let $\phi_{(\cdot)}: \mathbb{R} \rightarrow\left(\mathcal{H}_{0, n},\|\cdot\|_{0, n}\right), t \mapsto \phi_{t}$, be a $k$ times continuously differentiable map. Then the function $\phi: \mathbb{R}^{4} \times \mathbb{R} \rightarrow \mathbb{C}^{4}, \phi(x, t)=\phi_{t}(x)$, which is well-defined by Lemma 2.19, is $k$ times continuously differentiable. In particular, if $\phi(\cdot): \mathbb{R} \rightarrow \bigcap_{n \in \mathbb{N}_{0}} \mathcal{H}_{0, n}$ is smooth w.r.t. all norms $\|\cdot\|_{0, n}, n \in \mathbb{N}_{0}$, then the function $\phi$ is also smooth.

Proof. (a) Using (52), we write $T_{0}^{-y}=\mathcal{F}_{0 \mathcal{M}} T_{\mathcal{M}}^{-y} \mathcal{F}_{\mathcal{M} 0}=\mathcal{F}_{0 \mathcal{M}} e^{-i p y} \mathcal{F}_{\mathcal{M} 0}$ and $\partial^{\alpha}=(-i)^{|\alpha|} \mathcal{F}_{0 \mathcal{M}} p^{\alpha} \mathcal{F}_{\mathcal{M} 0}$. Because the operators $\mathcal{F}_{0 \mathcal{M}}:\left(\mathcal{H}_{\mathcal{M}, n},\|\cdot\|_{\mathcal{M}, n}\right) \rightarrow\left(\mathcal{H}_{0, n},\|\cdot\|_{0, n}\right)$ and $\mathcal{F}_{0 \mathcal{M}}:\left(\mathcal{H}_{0, n-l},\|\cdot\|_{0, n-l}\right) \rightarrow$ $\left(\mathcal{H}_{\mathcal{M}, n-l},\|\cdot\|_{\mathcal{M}, n-l}\right)$ are unitary, the claim is equivalent to showing that

$$
T_{\mathcal{M}}^{-}: \mathbb{R}^{4} \rightarrow \mathcal{B}\left(\left(\mathcal{H}_{\mathcal{M}, n},\|\cdot\|_{\mathcal{M}, n}\right),\left(\mathcal{H}_{\mathcal{M}, n-l},\|\cdot\|_{\mathcal{M}, n-l}\right)\right), \quad y \mapsto T_{\mathcal{M}}^{-y}
$$

is $l$ times continuously differentiable w.r.t. the strong operator topology with the derivatives

$$
\partial_{y}^{\alpha} T_{\mathcal{M}}^{-y}=(-i)^{|\alpha|} T_{\mathcal{M}}^{-y} p^{\alpha} .
$$

Convergence of the corresponding difference quotients in the strong operator topology is a consequence of the dominated convergence theorem, and the claim follows.

(b) The map $\Phi: \mathbb{R}^{4} \times \mathbb{R} \rightarrow\left(\mathcal{H}_{0, k+2},\|\cdot\|_{0, k+2}\right), \Phi(x, t)=T_{0}^{-x} \phi_{t}$ is $k$ times continuously partially differentiable w.r.t. the argument $t$. Furthermore, viewing $\Phi$ as a map $\Phi: \mathbb{R}^{4} \times \mathbb{R} \rightarrow$ $\left(\mathcal{H}_{0,2},\|\cdot\|_{0,2}\right)$, part (a) implies that all partial derivatives $\frac{\partial^{l}}{\partial t} \Phi(x, t), l=0, \ldots, k$, are $k$ times continuously differentiable w.r.t. to the argument $x$. Finally, using Lemma 2.19 and the fact $\phi=\delta_{0} \circ \Phi$ concludes the proof. 
Proof of Lemma 3.10. Claim (a) follows by induction over $l$, using Lemma 3.8 and taking derivatives w.r.t. $t$ of the right hand side of the differential equation (187). Claim (b) follows directly from part (a) using Lemma 3.11 (b).

\subsubsection{Proof of Theorem 2.23}

Proof of Theorem 2.23. The key to the claimed equivalence between the Schrödinger picture and the interaction picture is contained in the following calculation: Let $\phi: \mathbb{R}^{4} \times \mathbb{R} \rightarrow \mathbb{C}^{4}$ be a smooth function that solves the free Dirac equation in the first argument: $i \not_{x} \phi(x, t)=$ $m \phi(x, t)$. Let $\psi: \mathbb{R}^{4} \rightarrow \mathbb{C}^{4}$ be the smooth function given by $\psi(x)=\phi(x, \tau(x))$. Then we have the following equivalences for any $x \in \mathbb{R}^{4}$ :

$$
\begin{aligned}
& i \partial_{t} \phi(x, t)=v_{t}(x) h_{t}(x) A(x) \phi(x, t) \text { at } t=\tau(x) \\
& \Leftrightarrow \quad i h_{t}(x) v_{t}(x)^{-1} \partial_{t} \phi(x, t)=A(x) \phi(x, t) \text { at } t=\tau(x) \\
& \Leftrightarrow \quad i \phi_{y} \phi(x, \tau(y))=i \not_{y} \tau(y) \partial_{t} \phi(x, t)=A(y) \phi(x, \tau(y)) \text { at } y=x, t=\tau(y) \\
& \Leftrightarrow \quad\left(i \not \partial_{y}-A(y)-m\right) \phi(x, \tau(y))=-m \phi(x, \tau(y)) \text { at } y=x, t=\tau(y) \\
& \Leftrightarrow \quad\left(i \not \phi_{x}+i \not \phi_{y}-A(y)-m\right) \phi(x, \tau(y))=\left(i \not \partial_{x}-m\right) \phi(x, \tau(y))=0 \text { at } y=x, t=\tau(y) \\
& \Leftrightarrow \quad\left(i \not x_{x}-\not A(x)-m\right) \psi(x)=\left(i \not \phi_{x}-\not A(x)-m\right) \phi(x, \tau(x))=0 \\
& \Leftrightarrow \quad \psi \quad \psi \text { solves the Dirac equation (1) at } x \text { with potential } A \text {. }
\end{aligned}
$$

Here we used $\not^{2}=1$, Definition (83), and $\left(i \not \not_{x}-m\right) \phi(x, t)=0$ because $\phi_{t} \in \mathcal{C}_{0}$.

First, We prove part (a) of the theorem. Let $\psi \in \mathcal{C}_{A}$ and $\phi: \mathbb{R}^{4} \times \mathbb{R}$ be as in the hypothesis of the theorem. In particular, using smoothness and the support property of $\psi$, the function $\mathbb{R} \ni t \mapsto T_{\Sigma_{t}}^{-t e_{0}}\left(\left.\psi\right|_{\Sigma_{t}}\right)$ takes values in $\mathcal{C}_{\Sigma} \subseteq \mathcal{H}_{\Sigma, n}$ for any $n \in \mathbb{N}_{0}$ and is smooth with respect to the norm $\|\cdot\|_{\Sigma, n}$. Because $\mathcal{F}_{0 \Sigma}:\left(\mathcal{H}_{\Sigma, n},\|\cdot\|_{\Sigma, n}\right) \rightarrow\left(\mathcal{H}_{0, n},\|\cdot\|_{0, n}\right)$ is unitary, the function $\left.\mathbb{R} \ni t \mapsto \mathcal{F}_{0 \Sigma} T_{\Sigma_{t}}^{-t e_{0}} \psi\right|_{\Sigma_{t}}=\left.T_{0}^{-t e_{0}} \mathcal{F}_{0 \Sigma_{t}} \psi\right|_{\Sigma_{t}}=T_{0}^{-t e_{0}} \phi_{t} \in \mathcal{C}_{0} \subseteq \mathcal{H}_{0, n}$ is smooth w.r.t. the norm $\|\cdot\|_{0, n}$ as well. As this holds for all $n \in \mathbb{N}_{0}$, Lemma 3.11 (a) implies that $t \mapsto \phi_{t}=T_{0}^{t e_{0}} T_{0}^{-t e_{0}} \phi_{t} \in \mathcal{C}_{0} \subseteq \mathcal{H}_{0, n}$ is also smooth w.r.t. $\|\cdot\|_{0, n}$ for any $n \in \mathbb{N}_{0}$. Now Lemma 3.11 (b) shows that $\phi: \mathbb{R}^{4} \times \mathbb{R} \rightarrow \mathbb{C}^{4}$ is smooth. By definition, $\phi(x, t)$ solves the free Dirac equation in $x$-argument, fulfills the initial condition (84), and $\phi(x, \tau(x))=\psi(x)$ holds for all $x \in \mathbb{R}^{4}$. The direction " $\Leftarrow$ " in the sequence (194) of equivalences and the assumption $\psi \in \mathcal{C}_{A}$ implies

$$
\left.i\left(\partial_{t} \phi_{t}\right)\right|_{\Sigma_{t}}=v_{t} h_{t} A \mathcal{A} \mathcal{F}_{\Sigma_{t} 0} \phi_{t}
$$

Next we examine the support properties of all $\phi_{t}$ for $t$ in any given compact interval $I$. The set $K_{I}:=\operatorname{supp} \psi \cap \bigcup_{t \in I} \Sigma_{t}$ is compact, and for any $t \in I$, the function $\phi_{t}=\left.\mathcal{F}_{0 \Sigma_{t}} \psi\right|_{\Sigma_{t}}$ is supported in $\operatorname{supp}\left(\left.\psi\right|_{\Sigma_{t}}\right)+$ Causal $\subseteq K_{I}+$ Causal by Lemma 3.6. Because of $\phi_{t} \in \mathcal{C}_{0}$ for any $t$ and because the Dirac operator $i \not \phi_{x}-m$ in the $x$-argument commutes with the derivative $\partial_{t}$, this implies $\partial_{t} \phi(\cdot, t) \in \mathcal{C}_{0}$ for any $t \in \mathbb{R}$. Thus, we can rewrite (195) in the form $i \mathcal{F}_{\Sigma_{t} 0} \partial_{t} \phi_{t}=v_{t} h_{t} A \mathcal{F}_{\Sigma_{t} 0} \phi_{t}$. Because $\mathcal{F}_{\Sigma_{t} 0}: \mathcal{C}_{0} \rightarrow \mathcal{C}_{\Sigma_{t}}$ and $\mathcal{F}_{0 \Sigma_{t}}: \mathcal{C}_{\Sigma_{t}} \rightarrow \mathcal{C}_{0}$ are inverse to each other by Theorem 2.14, we conclude that formula (85) holds:

$$
i \partial_{t} \phi_{t}=\mathcal{F}_{0 \Sigma_{t}} v_{t} h_{t} A \mathcal{F}_{\Sigma_{t} 0} \phi_{t}=L_{t} \phi_{t}
$$


To finish the proof of part (a) of the theorem, we examine the support of $\psi_{t}$ uniformly in $t \in \mathbb{R}$. First, because the vector potential $A$ is compactly supported, we have $L_{t}=0$ for all $t \in \mathbb{R}$ with $|t|$ large enough. This shows that $\phi_{t}$ does not depend on $t$ for $t \geqslant t_{0}$ for some large enough $t_{1}>0$. The same holds for $t \leqslant t_{1}$ for some $t_{0}<0$ small enough. Using the compact interval $I=\left[t_{0}, t_{1}\right]$ we reconsider the compact set $K:=K_{I}$ from above. It follows $\operatorname{supp} \phi_{t} \subseteq K+$ Causal for all $t \in \mathbb{R}$, not only for $t \in I$. Thus, part (a) of the lemma is proven.

Next, We PROve PART (b). By the assumption supp $\phi \subseteq(K+$ Causal $) \times \mathbb{R}$, it follows $\operatorname{supp} \psi \subseteq K+$ Causal. Because $\phi$ fulfills the evolution equation (85), the direction " $\Rightarrow$ " in the sequence (194) of equivalences implies $\psi \in \mathcal{C}_{A}$. The remaining claims $\left.\psi\right|_{\Sigma}=\chi_{\Sigma}$ and $\phi_{t}=\left.\mathcal{F}_{0 \Sigma_{t}} \psi\right|_{\Sigma_{t}}$ follow immediately from the definitions.

\subsubsection{Proof of Theorem 2.20}

Proof of Theorem 2.20. We take a fixed future-directed foliation of space-time $\boldsymbol{\Sigma}$. Define $\chi:=\mathcal{F}_{0 \Sigma} \chi_{\Sigma}$. By Lemma 3.9 there is a solution $\phi_{(\cdot)}$ of the initial value problem (187).

First, WE PROVE THAT $\operatorname{supp} \phi_{t} \cap \Sigma_{t} \subseteq \operatorname{supp} \chi_{\Sigma}+$ Causal FOR ALL $t \in \mathbb{R}$. Let us assume $t \geqslant 0$. We define Causal $+:=\left\{x \in\right.$ Causal $\left.\mid x^{0} \geqslant 0\right\}$ as well as $\mathcal{C}_{0}\left(\chi_{\Sigma}, t\right):=\{\eta \in$ $\mathcal{C}_{0} \mid \operatorname{supp} \mathcal{F}_{\Sigma_{t} 0} \eta \subseteq \operatorname{supp} \chi_{\Sigma}+$ Causal $\left._{+}\right\}$and its closure $\mathcal{H}_{0, n}\left(\chi_{\Sigma}, t\right)$ in $\left(\mathcal{H}_{0, n},\|\cdot\|_{0, n}\right), n \in \mathbb{N}_{0}$. Furthermore, for all $T \geqslant 0$ we define

$$
X_{T, n}:=\left\{\varphi_{(\cdot)} \in \mathcal{C}\left([0, T],\left(\mathcal{H}_{0, n},\|\cdot\|_{0, n}\right)\right) \mid \forall 0 \leqslant s \leqslant T: \varphi_{s} \in \mathcal{H}_{0, n}\left(\chi_{\Sigma}, s\right)\right\}
$$

which is a Banach space w.r.t. the norm $\left\|\varphi_{(\cdot)}\right\|_{X_{T, n}}:=\sup _{t \in[0, T]}\left\|\varphi_{t}\right\|_{0, n}$. As seen in the proof of Lemma 3.9, for any given $T \geqslant 0$ and $n \in \mathbb{N}_{0}$ the Volterra integral equation (188) gives rise to a self-map $S: \mathcal{C}\left([0, T],\left(\mathcal{H}_{0, n},\|\cdot\|_{0, n}\right)\right) \smile$

$$
S: \varphi_{(\cdot)} \mapsto\left(t \mapsto S_{t}\left(\varphi_{(\cdot)}\right):=\mathcal{F}_{0 \Sigma} \chi_{\Sigma}-i \int_{0}^{t} L_{s} \varphi_{s} d s\right), \quad \text { for } \varphi_{(\cdot)} \in \mathcal{C}\left([0, T],\left(\mathcal{H}_{0, n},\|\cdot\|_{0, n}\right)\right)
$$

We further claim that $S: X_{T, n} \oslash$. To see this we need to show $S_{t}\left(\varphi_{(\cdot)}\right) \in \mathcal{H}_{0, n}\left(\chi_{\Sigma}, t\right)$ for all $\varphi_{(\cdot)} \in X_{T, n}$ and $0 \leqslant t \leqslant T$. First, thanks to Theorem 2.14 (b), the fact that $\Sigma$ is future oriented, and $\mathrm{Causal}_{+}+\mathrm{Causal}_{+}=\mathrm{Causal}_{+}$, one has $\mathcal{C}_{0}\left(\chi_{\Sigma}, s\right) \subseteq \mathcal{C}_{0}\left(\chi_{\Sigma}, t\right)$ for all $0 \leqslant s \leqslant t$. This implies that $\mathcal{F}_{0 \Sigma} \chi_{\Sigma} \in \mathcal{C}_{0}\left(\chi_{\Sigma}, t\right)$ for all $0 \leqslant t \leqslant T$. Second, with the help of the representation of $L_{s}$ given in (85) and using Theorem 2.14 (a), we observe that $L_{s} \eta \in \mathcal{C}_{0}\left(\chi_{\Sigma}, s\right)$ for any $\eta \in \mathcal{C}_{0}\left(\chi_{\Sigma}, s\right)$ and $0 \leqslant s \leqslant T$. Using that $L_{t}:\left(\mathcal{H}_{0, n},\|\cdot\|_{0, n}\right) \wp$ is bounded, which was proven in Lemma 3.8, and that $\mathcal{C}_{0}\left(\chi_{\Sigma}, s\right)$ is dense in $\mathcal{H}_{0, n}\left(\chi_{\Sigma}, s\right)$ w.r.t. $\|\cdot\|_{0, n}$, we conclude $L_{s} \varphi_{s} \in \mathcal{H}_{0, n}\left(\chi_{\Sigma}, s\right) \subseteq \mathcal{H}_{0, n}\left(\chi_{\Sigma}, t\right)$ for any $\varphi_{(\cdot)} \in \mathcal{H}_{0, n}\left(\chi_{\Sigma}, s\right)$ and $0 \leqslant s \leqslant t \leqslant T$. This proves $S: X_{T, n} \bigcirc$.

In consequence, the corresponding unique fixed-point $\phi_{(\cdot)}$ found in Lemma 3.9 fulfills $\phi_{(\cdot)} \in X_{T, n}$. In particular, all $\left.\phi_{t}\right|_{\Sigma_{t}}, t \geqslant 0$, are supported in $\left(\operatorname{supp} \chi_{\Sigma}+\right.$ Causal $\left._{+}\right) \cap \Sigma_{t}$. This together with an analogous argument for $t \leqslant 0$ implies $\left.\operatorname{supp} \phi_{t}\right|_{\Sigma_{t}} \subseteq \operatorname{supp} \chi_{\Sigma}+$ Causal for $t \in \mathbb{R}$. 
PART (i). From part (b) of Lemma 3.10 we know that $\phi_{(\cdot)}$ is smooth. Hence, part (b) of Theorem 2.23 implies that the function $\psi$ given by $\psi(x):=\phi(x, \tau(x))$ is in $\mathcal{C}_{A}$ with $\left.\psi\right|_{\Sigma}=\chi_{\Sigma}$. Furthermore, we have supp $\psi \subseteq \operatorname{supp} \chi_{\Sigma}+$ Causal.

PART (ii). Let $\tilde{\psi} \in \mathcal{C}^{\infty}\left(\mathbb{R}^{4}, \mathbb{C}^{4}\right)$ solve the Dirac equation (1) for $\left.\tilde{\psi}\right|_{\Sigma}=\chi_{\Sigma}$. Suppose $\widetilde{\psi} \in \mathcal{C}_{A}$. Then, by part (a) of Theorem 2.23 together with the uniqueness statement of Lemma 3.9 we get $\widetilde{\psi}=\psi$. Finally, we show that $\tilde{\psi} \in \mathcal{C}_{A}$. For this we use a duality argument. For any Cauchy surface $\Sigma^{\prime}$ and $\phi \in \mathcal{C}_{A}$ the pairing $\langle\phi, \widetilde{\psi}\rangle_{\Sigma^{\prime}}:=\int_{\Sigma^{\prime}} \overline{\phi(x)} i_{\gamma}\left(d^{4} x\right) \widetilde{\psi}(x)$, cf. (15), is well-defined because the integrand is smooth and has compact support, just as $\left.\phi\right|_{\Sigma^{\prime}}$. Recalling (17), we find $d\left[\overline{\phi(x)} i_{\gamma}\left(d^{4} x\right) \widetilde{\psi}(x)\right]=0$ so that for all Cauchy surfaces $\Sigma^{\prime}$ we have $\langle\phi, \widetilde{\psi}\rangle_{\Sigma}=\langle\phi, \widetilde{\psi}\rangle_{\Sigma^{\prime}}$. For any $\varphi_{\Sigma^{\prime}} \in \mathcal{C}_{\Sigma^{\prime}}$ with $\operatorname{supp} \varphi_{\Sigma^{\prime}} \cap\left(\operatorname{supp} \chi_{\Sigma}+\right.$ Causal $)=\varnothing$ one has $\left(\operatorname{supp} \varphi_{\Sigma^{\prime}}+\right.$ Causal $) \cap \operatorname{supp} \chi_{\Sigma}=\varnothing$. Consider $\phi \in \mathcal{C}_{A}$ with $\left.\phi\right|_{\Sigma^{\prime}}=\varphi_{\Sigma^{\prime}}$ the existence of which is ensured by part (i). Then by part (i) we know that $\operatorname{supp} \phi \subseteq\left(\operatorname{supp} \varphi_{\Sigma^{\prime}}+\right.$ Causal), and hence, $\operatorname{supp} \phi \cap \operatorname{supp} \chi_{\Sigma}=\varnothing$. We conclude

$$
\int_{\Sigma^{\prime}} \overline{\varphi_{\Sigma^{\prime}}(x)} i_{\gamma}\left(d^{4} x\right) \tilde{\psi}(x)=\langle\phi, \widetilde{\psi}\rangle_{\Sigma^{\prime}}=\langle\phi, \widetilde{\psi}\rangle_{\Sigma}=\int_{\Sigma} \overline{\phi(x)} i_{\gamma}\left(d^{4} x\right) \chi_{\Sigma}(x)=0
$$

as $\left.\tilde{\psi}\right|_{\Sigma}=\chi_{\Sigma}$. Since $\tilde{\psi}$ is continuous we conclude that supp $\tilde{\psi} \subseteq \operatorname{supp} \chi_{\Sigma}+$ Causal. Therefore, $\tilde{\psi} \in \mathcal{C}_{A}$.

\subsubsection{Proof of Theorem 2.22}

Proof of Theorem 2.22. Theorem 2.20 implies: For any Cauchy surface $\Sigma$ and any vector potential $A \in C_{c}^{\infty}\left(\mathbb{R}^{4}, \mathbb{R}^{4}\right)$, the restriction map $\mathcal{F}_{\Sigma A}: \mathcal{C}_{A} \rightarrow \mathcal{C}_{\Sigma}, \mathcal{F}_{\Sigma A} \psi=\left.\psi\right|_{\Sigma}$ is a bijection. Let $\mathcal{F}_{A \Sigma}: \mathcal{C}_{\Sigma} \rightarrow \mathcal{C}_{A}$ denote its inverse. Moreover, by the definition of the scalar product on $\mathcal{C}_{A}$ given in (15), see also the argument (17), the restriction map $\mathcal{F}_{\Sigma A}: \mathcal{C}_{A} \rightarrow \mathcal{C}_{\Sigma}$ is isometric. Taking the closure of this map, it has a unitary extension $\mathcal{F}_{\Sigma A}: \mathcal{H}_{A} \rightarrow \mathcal{H}_{\Sigma}$ with a unitary inverse $\mathcal{F}_{A \Sigma}: \mathcal{H}_{\Sigma} \rightarrow \mathcal{H}_{A}$. The operator $\mathcal{F}_{\Sigma^{\prime} \Sigma}^{A}:=\mathcal{F}_{\Sigma^{\prime} A} \mathcal{F}_{A \Sigma}: \mathcal{H}_{\Sigma} \rightarrow \mathcal{H}_{\Sigma^{\prime}}$ is then the unique unitary extension of the isometric bijection $\mathcal{F}_{\Sigma^{\prime} \Sigma}^{A}=\mathcal{F}_{\Sigma^{\prime} A} \mathcal{F}_{A \Sigma}: \mathcal{C}_{\Sigma} \rightarrow \mathcal{C}_{\Sigma^{\prime}}$.

\section{A Auxiliary Results}

In this appendix, we prove some of the technical lemmas used in the rest of the paper. The first lemma deals with the inequalities controlling the geometry of the complexified mass shell.

Lemma A.1 (Geometric Properties of $\left.\mathcal{M}_{\mathbb{C}}\right)$. For $p=\left(p^{0}, \mathbf{p}\right) \in \mathcal{M}_{\mathbb{C}}$, one has the inequalities

$$
\begin{aligned}
& \left|\operatorname{Im} p^{0}\right| \leqslant|\operatorname{Im} \mathbf{p}|, \\
& |\operatorname{Im} \mathbf{p}| \leqslant|\operatorname{Im} p| \leqslant \sqrt{2}|\operatorname{Im} \mathbf{p}|, \\
& m \leqslant|p| \leqslant \sqrt{3}(m \vee|\mathbf{p}|), \\
& m \vee|\mathbf{p}| \leqslant C_{16}\left(\frac{m}{12} \vee\left|p^{0}\right|\right) e^{\epsilon|\operatorname{Im} \mathbf{p}|} \quad \text { for } \epsilon>0,
\end{aligned}
$$

with a constant $C_{16}=C_{16}(\epsilon m)>0$. 
Proof. For the first claim (200), we calculate with the notation $\mathbf{p}^{2}=\left(p^{1}\right)^{2}+\left(p^{2}\right)^{2}+\left(p^{3}\right)^{2}$ :

$$
\begin{aligned}
& 2\left|\operatorname{Im} p^{0}\right|^{2}=\left|p^{0}\right|^{2}-\operatorname{Re}\left(\left(p^{0}\right)^{2}\right)=\left|\mathbf{p}^{2}+m^{2}\right|-\operatorname{Re}\left(\mathbf{p}^{2}+m^{2}\right) \\
& \leqslant\left|\mathbf{p}^{2}\right|+m^{2}-\operatorname{Re}\left(\mathbf{p}^{2}\right)-m^{2}=\left|\mathbf{p}^{2}\right|-\operatorname{Re}\left(\mathbf{p}^{2}\right) \leqslant|\mathbf{p}|^{2}-\operatorname{Re}\left(\mathbf{p}^{2}\right)=2|\operatorname{Im} \mathbf{p}|^{2} .
\end{aligned}
$$

This also yields claim (201):

$$
|\operatorname{Im} \mathbf{p}|^{2} \leqslant|\operatorname{Im} p|^{2}=\left|\operatorname{Im} p^{0}\right|^{2}+|\operatorname{Im} \mathbf{p}|^{2} \leqslant 2|\operatorname{Im} \mathbf{p}|^{2}
$$

The inequality on the right in Claim (202) follows directly from

$$
|p|^{2}=\left|p^{0}\right|^{2}+|\mathbf{p}|^{2}=\left|\mathbf{p}^{2}+m^{2}\right|+|\mathbf{p}|^{2} \leqslant 2|\mathbf{p}|^{2}+m^{2} \leqslant 3\left(|\mathbf{p}|^{2} \vee m^{2}\right) .
$$

The bound $m \leqslant|p|$ is a consequence of $m^{2}=\left|p^{2}\right| \leqslant|p|^{2}$. To finally prove (203), we observe that $\mathbf{p}^{2}+m^{2}=\left(p^{0}\right)^{2}$ implies

$$
|\mathbf{p}|^{2}-2|\operatorname{Im} \mathbf{p}|^{2}+m^{2}=\operatorname{Re}\left(\mathbf{p}^{2}\right)+m^{2}=\operatorname{Re}\left(\left(p^{0}\right)^{2}\right) \leqslant\left|p^{0}\right|^{2},
$$

and hence, $|\mathbf{p}|^{2}+m^{2} \leqslant\left|p^{0}\right|^{2}+2|\operatorname{Im} \mathbf{p}|^{2}$. We get $m^{2} \vee|\mathbf{p}|^{2} \leqslant|\mathbf{p}|^{2}+m^{2} \leqslant 3\left(\left|p^{0}\right|^{2} \vee|\operatorname{Im} \mathbf{p}|^{2}\right)$ and thus

$$
m \vee|\mathbf{p}| \leqslant \sqrt{3}\left(\left|p^{0}\right| \vee|\operatorname{Im} \mathbf{p}|\right) \leqslant C_{16}\left(\frac{m}{12} \vee\left|p^{0}\right|\right)(1+\epsilon|\operatorname{Im} \mathbf{p}|) \leqslant C_{16}\left(\frac{m}{12} \vee\left|p^{0}\right|\right) e^{\epsilon|\operatorname{Im} \mathbf{p}|}
$$

with a constant $C_{16}=C_{16}(\epsilon m)>0$.

Proof of Lemma 3.2. Heuristically, the idea is to choose $k(t)=\left(k^{0}(t), \mathbf{k}(t)\right)$ by a Euler substitution as a rational function of $t$ such that $\mathbf{k}^{\prime}(0)$ is small whenever $p^{0}$ is close to 0 . More precisely, we proceed as follows. We abbreviate $\delta:=1 / 12, \epsilon:=1 / 3$, and $\gamma=1 / 6$. The only facts that we need about these positive constants are the following:

$$
\begin{array}{r}
H:=2\left(\sqrt{1-\delta^{2}}-\epsilon-\delta\right)>0, \\
\gamma \geqslant \frac{\epsilon(\epsilon+2 \delta)}{H}, \\
\epsilon-2 \delta-\gamma \geqslant 0 .
\end{array}
$$

Given $p=\left(p^{0}, \mathbf{p}\right) \in \mathcal{M}_{\mathbb{C}}$ with

$$
\left|p^{0}\right| \leqslant \delta m
$$

we observe

$$
|\mathbf{p}| \geqslant m \sqrt{1-\delta^{2}}>0
$$

from

$$
|\mathbf{p}|^{2} \geqslant\left|\mathbf{p}^{2}\right|=\left|\left(p^{0}\right)^{2}-m^{2}\right| \geqslant m^{2}-\left|p^{0}\right|^{2} \geqslant m^{2}\left(1-\delta^{2}\right) .
$$

We set $\mathbf{q}=\left(q^{1}, q^{2}, q^{3}\right):=\mathbf{p}^{*} /|\mathbf{p}|$, where $\mathbf{p}^{*}$ denotes the complex conjugate of $\mathbf{p}$, and take $r \in \mathbb{C}$ with $r^{2}=\mathbf{q}^{2}$. It fulfills $|r| \leqslant 1$ because of $|r|^{2}=\left|\mathbf{q}^{2}\right| \leqslant|\mathbf{q}|^{2}=1$. We set for $t \in \mathbb{C}$ :

$$
h(t):=2\left(\mathbf{p q}-\epsilon m r t-p^{0} r\right)=2\left(|\mathbf{p}|-\epsilon m r t-p^{0} r\right),
$$


where we have abbreviated pq $:=\sum_{j=1}^{3} p^{j} q^{j}$. Using (213), $|r| \leqslant 1,(212)$, and (209), we get the following for $t \in \bar{\Delta}$.

$$
|h(t)| \geqslant 2\left(|\mathbf{p}|-\epsilon m-\left|p^{0}\right|\right) \geqslant m H>0
$$

In particular,

$$
g: \bar{\Delta} \rightarrow \mathbb{C}, \quad g(t):=\frac{(\epsilon m t)^{2}+2 p^{0} \epsilon m t}{h(t)}
$$

is well-defined and extends to a holomorphic function in a neighborhood of $\bar{\Delta}$. Using (210), it fulfills the following bound for $t \in \bar{\Delta}$.

$$
|g(t)| \leqslant m \frac{\epsilon(\epsilon+2 \delta)}{H} \leqslant \gamma m
$$

We introduce $k(t)=\left(k^{0}(t), \mathbf{k}(t)\right)$ for $t \in \bar{\Delta}$ by

$$
\begin{aligned}
k^{0}(t) & :=p^{0}+\epsilon m t+g(t) r \\
\mathbf{k}(t) & :=\mathbf{p}+g(t) \mathbf{q}
\end{aligned}
$$

Note that $k$ also extends to a holomorphic function in a neighborhood of $\bar{\Delta}$. Expanding the squares and using $r^{2}=\mathbf{q}^{2}$ and $\left(p^{0}\right)^{2}-\mathbf{p}^{2}=m^{2}$ we observe that $k(t) \in \mathcal{M}_{\mathbb{C}}$ as

$$
k^{0}(t)^{2}-\mathbf{k}(t)^{2}=\left(p^{0}\right)^{2}-\mathbf{p}^{2}+g(t)^{2} r^{2}-g(t)^{2} \mathbf{q}^{2}+(\epsilon m t)^{2}+2 p^{0} \epsilon m t-g(t) h(t)=m^{2} .
$$

The claim $k(0)=p$ follows from $g(0)=0$. To obtain claim (100), we estimate for $t \in \bar{\Delta}$ :

$$
|\mathbf{k}(t)-\mathbf{p}|=|g(t) \mathbf{q}|=|g(t)| \leqslant \gamma m .
$$

Finally, claim (101) for $t \in \mathbb{C}$ with $|t|=1$ is obtained from (212), (218), $|r| \leqslant 1$, and (211) as follows.

$$
\left|k^{0}(t)\right| \geqslant|\epsilon m t|-\left|p^{0}\right|-|g(t) r| \geqslant \epsilon m-\delta m-\gamma m \geqslant \delta m
$$

Proof of Lemma 2.11. It is obvious that $T_{\Sigma}^{-y}$ maps $\mathcal{C}_{\Sigma}$ to $\mathcal{C}_{\Sigma-y}, T_{A}^{-y}$ maps $\mathcal{C}_{A}$ to $\mathcal{C}_{A(\cdot+y)}$, and $L_{\Sigma}^{(S, \Lambda)} \operatorname{maps} \mathcal{C}_{\Sigma}$ to $\mathcal{C}_{\Lambda \Sigma}$

To see that $T_{\mathcal{M}}^{-y}$ maps $\mathcal{C}_{\mathcal{M}}$ to $\mathcal{C}_{\mathcal{M}}$, we consider $\psi \in \mathcal{C}_{\mathcal{M}}$, its holomorphic extension $\Psi$ : $\mathcal{M}_{\mathbb{C}} \rightarrow \mathbb{C}^{4}$ and $\alpha>0$ with $\|\psi\|_{\mathcal{M}, \alpha, n}<\infty$ for all $n \in \mathbb{N}$. Using inequality (201), we conclude for any $n \in \mathbb{N}$

$$
\left\|T_{\mathcal{M}}^{-y} \psi\right\|_{\mathcal{M}, \alpha+\sqrt{2}|y|, n}=\sup _{p \in \mathcal{M}_{\mathbb{C}}}|p|^{n-1} e^{-(\alpha+\sqrt{2}|y|)|\operatorname{Im} \mathbf{p}|} e^{y \operatorname{Im} p}|\Psi(p)| \leqslant\|\psi\|_{\mathcal{M}, \alpha, n}<\infty
$$

This proves $T_{\mathcal{M}}^{-y} \psi \in \mathcal{C}_{\mathcal{M}}$.

To show that $L_{A}^{(S, \Lambda)}$ maps $\mathcal{C}_{A}$ to $\mathcal{C}_{\Lambda A\left(\Lambda^{-1} .\right)}$, we take any $\psi \in \mathcal{C}_{A}$ and a compact set $K \subset \mathbb{R}^{4}$ with $\operatorname{supp} \psi \subseteq K+$ Causal. Because of $\Lambda$ Causal $=$ Causal, we infer $\operatorname{supp}\left(L_{A}^{(S, \Lambda)} \psi\right) \subseteq$ $\Lambda K+$ Causal, and $\Lambda K$ is compact. Furthermore, $\psi^{\prime}:=L_{A}^{(S, \Lambda)} \psi$ is smooth and solves the 
Dirac equation subject to the transformed external potential. To see this, using the notation $x^{\prime}=\Lambda x, \partial_{\mu}^{\prime}=\partial / \partial x^{\prime \mu}$, and $\partial_{\nu}=\partial / \partial x^{\nu}$, we note

$$
m \psi^{\prime}\left(x^{\prime}\right)=S m \psi(x)=S \gamma^{\nu}\left(i \partial_{\nu}-A_{\nu}\left(\Lambda^{-1} x^{\prime}\right)\right) \psi(x)
$$

Formula (42) and the identity $\delta^{\mu}{ }_{\nu}=\Lambda_{\sigma}{ }^{\mu} \Lambda^{\sigma}{ }_{\nu}$ implies $S \gamma^{\nu}=\Lambda_{\mu}{ }^{\nu} \gamma^{\mu} S$, and therefore

$$
\begin{aligned}
(225) & =\Lambda_{\mu}{ }^{\nu} \gamma^{\mu} S\left(i \partial_{\nu}-A_{\nu}\left(\Lambda^{-1} x^{\prime}\right)\right) \psi(x) \\
& =\gamma^{\mu}\left(i \partial_{\mu}^{\prime}-\Lambda_{\mu}{ }^{\nu} A_{\nu}\left(\Lambda^{-1} x^{\prime}\right)\right) \psi^{\prime}\left(x^{\prime}\right),
\end{aligned}
$$

where we have use $\partial_{\mu}^{\prime}=\Lambda_{\mu}{ }^{\nu} \partial_{\nu}$. This shows $\psi^{\prime} \in \mathcal{C}_{\Lambda A\left(\Lambda^{-1}\right)}$.

Finally, to see that $L_{\mathcal{M}}^{(S, \Lambda)}$ maps $\mathcal{C}_{\mathcal{M}}$ to $\mathcal{C}_{\mathcal{M}}$, we take $\phi \in \mathcal{C}_{\mathcal{M}}$, its holomorphic extension $\Phi: \mathcal{M}_{\mathbb{C}} \rightarrow \mathbb{C}^{4}$, any vector $p^{\prime}=\left(p^{\prime 0}, \mathbf{p}^{\prime}\right) \in \mathcal{M}_{\mathbb{C}}, \alpha>0$ with $\|\psi\|_{\mathcal{M}, \alpha, n}<\infty$ for all $n \in \mathbb{N}$, and set $\alpha^{\prime}=\sqrt{2}\left\|\Lambda^{-1}\right\| \alpha$ and $p=\left(p^{0}, \mathbf{p}\right)=\Lambda^{-1} p^{\prime} \in \mathcal{M}_{\mathbb{C}}$. We get the following with inequality (201): $\alpha^{\prime}\left|\operatorname{Im} \mathbf{p}^{\prime}\right| \geqslant \alpha^{\prime}\left|\operatorname{Im} p^{\prime}\right| / \sqrt{2} \geqslant \alpha^{\prime}|| \Lambda^{-1} \|^{-1}|\operatorname{Im} p| / \sqrt{2} \geqslant \alpha|\operatorname{Im} \mathbf{p}|$, and hence, for all $n \in \mathbb{N}$ :

$$
\left|p^{\prime}\right|^{n-1} e^{-\alpha^{\prime}\left|\operatorname{Im} \mathbf{p}^{\prime}\right|}\left|S \Phi\left(\Lambda^{-1} p^{\prime}\right)\right| \leqslant\|S\|\|\Lambda\|^{n-1}|p|^{n-1} e^{-\alpha|\operatorname{Im} p|}|\Phi(p)| \leqslant\|S\|\|\Lambda\|^{n-1}\|\phi\|_{\mathcal{M}, \alpha, n},
$$

using Definition (32). This proves $\left\|L_{\mathcal{M}}^{(S, \Lambda)} \phi\right\|_{\mathcal{M}, \alpha^{\prime}, n}<\infty$ and therefore $L_{\mathcal{M}}^{(S, \Lambda)} \phi \in \mathcal{C}_{\mathcal{M}}$. It is obvious that the six maps (50)- (55) are invertible with inverses $T_{\Sigma}^{y}, T_{A}^{y}, T_{\mathcal{M}}^{y}, L_{\Sigma}^{\left(S^{-1}, \Lambda^{-1}\right)}$, $L_{A}^{\left(S^{-1}, \Lambda^{-1}\right)}$, and $L_{\mathcal{M}}^{\left(S^{-1}, \Lambda^{-1}\right)}$, respectively. Furthermore, they are isometric. This is obvious in the case of the three translation maps (50)- (52). We consider now Lorentz transformations (53)-(55). For $\phi, \psi \in \mathcal{C}_{\Sigma}, \phi^{\prime}=L_{\Sigma}^{\left(S^{-1}, \Lambda^{-1}\right)} \phi$, and $\psi^{\prime}=L_{\Sigma}^{\left(S^{-1}, \Lambda^{-1}\right)} \psi$ we get by (10), (41), and the invariance relation (48):

$$
\begin{aligned}
\left\langle\phi^{\prime}, \psi^{\prime}\right\rangle & =\int_{\Lambda \Sigma} \overline{\phi^{\prime}\left(x^{\prime}\right)} i_{\gamma}\left(d^{4} x^{\prime}\right) \psi^{\prime}\left(x^{\prime}\right)=\int_{\Lambda \Sigma} \overline{S \phi\left(\Lambda^{-1} x^{\prime}\right)} i_{\gamma}\left(d^{4} x^{\prime}\right) S \psi\left(\Lambda^{-1} x^{\prime}\right) \\
& =\int_{\Lambda \Sigma} \overline{\phi\left(\Lambda^{-1} x^{\prime}\right)} \gamma^{0} S^{*} \gamma^{0} i_{\gamma}\left(d^{4} x^{\prime}\right) S \psi\left(\Lambda^{-1} x^{\prime}\right)=\int_{\Lambda \Sigma} \overline{\phi\left(\Lambda^{-1} x^{\prime}\right)} S^{-1} i_{\gamma}\left(d^{4} x^{\prime}\right) S \psi\left(\Lambda^{-1} x^{\prime}\right) \\
& =\int_{\Sigma} \overline{\phi(x)} i_{\gamma}\left(d^{4} x\right) \psi(x)=\langle\phi, \psi\rangle
\end{aligned}
$$

Using (15), the same calculation is valid for $\phi, \psi \in \mathcal{C}_{A}, \phi^{\prime}=L_{A}^{\left(S^{-1}, \Lambda^{-1}\right)} \phi$, and $\psi^{\prime}=L_{A}^{\left(S^{-1}, \Lambda^{-1}\right)} \psi$.

For $\phi, \psi \in \mathcal{C}_{\mathcal{M}}, \phi^{\prime}=L_{\mathcal{M}}^{\left(S^{-1}, \Lambda^{-1}\right)} \phi$, and $\psi^{\prime}=L_{\mathcal{M}}^{\left(S^{-1}, \Lambda^{-1}\right)} \psi$, the fact $m_{\Lambda} \mathcal{M}=\mathcal{M}$, equations (30), (41), and the invariance relation (49) yield

$$
\begin{aligned}
& \left\langle\phi^{\prime}, \psi^{\prime}\right\rangle=\int_{\mathcal{M}} \overline{S \phi\left(\Lambda^{-1} p^{\prime}\right)} S \psi\left(\Lambda^{-1} p^{\prime}\right) \frac{i_{p^{\prime}}\left(d^{4} p^{\prime}\right)}{m}=\int_{\mathcal{M}} \overline{\phi\left(\Lambda^{-1} p^{\prime}\right)} \psi\left(\Lambda^{-1} p^{\prime}\right) \frac{i_{p^{\prime}}\left(d^{4} p^{\prime}\right)}{m} \\
& =\int_{\mathcal{M}} \overline{\phi(p)} \psi(p) \frac{i_{p}\left(d^{4} p\right)}{m}=\langle\phi, \psi\rangle .
\end{aligned}
$$

Since the six maps (50)-(55) are isometric bijections, it follows that they extend to unitary maps on the respective Hilbert spaces. 
Proof of Lemma 2.13. First, we show that for a given $\lambda \in \mathcal{C}_{c}^{\infty}\left(\mathbb{R}^{4}, \mathbb{R}\right)$ the multiplication operator $\Gamma_{\lambda}$ maps $\mathcal{C}_{A}$ to $\mathcal{C}_{A+\partial \lambda}$. To show this, we take a $\psi \in \mathcal{C}_{A}$ and define $\psi^{\prime}(x):=\Gamma_{\lambda} \psi(x)=$ $e^{-i \lambda(x)} \psi(x)$ for $x \in \mathbb{R}^{4}$. Clearly, $\operatorname{supp} \psi^{\prime}=\operatorname{supp} \psi$ and $\psi^{\prime} \in \mathcal{C}^{\infty}\left(\mathbb{R}^{4}, \mathbb{C}^{4}\right)$. The wave function $\psi^{\prime}$ fulfills the Dirac equation subject to the transformed potential $A^{\prime}:=A+\partial \lambda$ :

$$
\begin{aligned}
\left(i \not \partial-A^{\prime}(x)\right) \psi^{\prime}(x) & =\left(i \not \partial-A^{\prime}(x)\right) e^{-i \lambda(x)} \psi(x) \\
& =e^{-i \lambda(x)}(i \not \partial-A(x)) \psi(x) \\
& =e^{-i \lambda(x)} m \psi(x)=m \psi^{\prime}(x) .
\end{aligned}
$$

It is obvious that the map (57) is invertible and isometric. Therefore, it extends uniquely to a unitary map $\Gamma_{\lambda}: \mathcal{H}_{A} \rightarrow \mathcal{H}_{A+\partial \lambda}$.

Proof of Theorem 2.15. Equations (64) and (65) are obvious. To prove (66), let $\psi \in \mathcal{C}_{\Sigma}$, $\psi^{\prime}=L_{\Sigma}^{(S, \Lambda)} \psi$, and $p^{\prime}=\Lambda p \in \mathcal{M}$. Using the invariance relation (48) and the consequence $\not p^{\prime}=S \not p S^{-1}$ of (41) and (42), we obtain

$$
\begin{aligned}
{\left[\mathcal{F}_{\mathcal{M}, \Lambda \Sigma} L_{\Sigma}^{(S, \Lambda)} \psi\right]\left(p^{\prime}\right) } & =\frac{\not p^{\prime}+m}{2 m}(2 \pi)^{-3 / 2} \int_{\Lambda \Sigma} e^{i p^{\prime} x^{\prime}} i_{\gamma}\left(d^{4} x^{\prime}\right) \psi^{\prime}\left(x^{\prime}\right) \\
& =S \frac{\not p+m}{2 m}(2 \pi)^{-3 / 2} \int_{\Lambda \Sigma} e^{i p^{\prime} x^{\prime}} S^{-1} i_{\gamma}\left(d^{4} x^{\prime}\right) S \psi\left(\Lambda^{-1} x^{\prime}\right) \\
& =S \frac{\not p+m}{2 m}(2 \pi)^{-3 / 2} \int_{\Sigma} e^{i p x} i_{\gamma}\left(d^{4} x\right) \psi(x) \\
& =\left[L_{\mathcal{M}}^{(S, \Lambda)} \mathcal{F}_{\mathcal{M} \Sigma} \psi\right]\left(p^{\prime}\right) .
\end{aligned}
$$

Finally, equation (67) is an immediate consequence of the invariance relation (49).

The next auxiliary lemma deals with multiplication operators in the Sobolev space $\mathcal{H}_{\Sigma, n}$. Let $\Sigma$ be a Cauchy surface and $K$ an open and relatively compact subset of $\mathbb{R}^{3}$. Given $n \in \mathbb{N}_{0}$ we endow $\mathcal{C}_{c}^{n}\left(K, \mathbb{C}^{4 \times 4}\right)$ with the norm

$$
\|Z\|_{K, n, \infty}:=\sum_{\substack{\beta \in \mathbb{N}_{0}^{3} \\|\beta| \leqslant n}} \sup _{\mathbf{x} \in K}\left|D^{\beta} Z\left(t_{\Sigma}(\mathbf{x}), \mathbf{x}\right)\right|, \quad \text { where } \quad D^{\beta}=D_{1}^{\beta_{1}} D_{2}^{\beta_{2}} D_{3}^{\beta_{3}} .
$$

Lemma A.2. There is a well-defined bounded linear map

$$
\left(\mathcal{C}_{c}^{n}\left(K, \mathbb{C}^{4 \times 4}\right),\|\cdot\|_{K, n, \infty}\right) \rightarrow\left(\mathcal{B}\left(\mathcal{H}_{\Sigma, n},\|\cdot\|_{\Sigma, n}\right),\|\cdot\|_{\mathcal{H}_{\Sigma, n} \rightarrow \mathcal{H}_{\Sigma, n}}\right), \quad Z \mapsto(\psi \mapsto Z \psi)
$$

Proof. For any $j=0,1,2,3$ and using (77) and (78) we compute

$$
\begin{aligned}
& i \partial_{j} Z \psi=\sum_{k=1}^{3} \alpha_{j k}^{\Sigma} D_{k}(Z \psi)+\beta_{j}^{\Sigma} Z \psi \\
& =\sum_{k=1}^{3} \alpha_{j k}^{\Sigma} Z \partial_{k} \psi+\sum_{k=1}^{3} \alpha_{j k}^{\Sigma} \frac{\partial t_{\Sigma}}{\partial x^{k}} Z \partial_{0} \psi+\left[\sum_{k=1}^{3} \alpha_{j k}^{\Sigma}\left(D_{k} Z\right)+\beta_{j}^{\Sigma} Z\right] \psi=: \sum_{\substack{\alpha \in \mathbb{N}_{0}^{4} \\
|\alpha| \leqslant 1}} M_{\beta, \alpha} \partial^{\alpha} \psi
\end{aligned}
$$


with $\beta=\left(\delta_{i j}\right)_{i=0,1,2,3}$. Iterating this formula for a general multi-index $\beta$, with $Z$ replaced by $M_{\beta, \alpha}$ and with $\psi$ replaced by $\partial^{\alpha} \psi$ in the induction step, yields

$$
i^{|\beta|} \partial^{\beta} Z \psi=\sum_{\substack{\alpha \in \mathbb{N}_{0}^{4} \\|\alpha| \leqslant n}} M_{\beta, \alpha} \partial^{\alpha} \psi, \quad\left\|\partial^{\beta} Z \psi\right\| \leqslant \sum_{\substack{\alpha \in \mathbb{N}_{0}^{4} \\|\alpha| \leqslant n}}\left\|M_{\beta, \alpha}\right\|_{\infty}\left\|\partial^{\alpha} \psi\right\|,
$$

for $\beta \in \mathbb{N}_{0}^{4},|\beta| \leqslant n$, with some $\mathbb{C}^{4 \times 4}$-valued continuous functions $M_{\beta, \alpha}$ compactly supported in $K$ and depending linearly on $D^{\gamma} Z,|\gamma| \leqslant n$. Taking the square and summing over $|\beta| \leqslant n$ yields the claim.

\section{References}

[BGP07] C. Bär and N. Ginoux and F. Pfäffle Wave Equations on Lorentzian Manifolds and Quantization. European Mathematical Society, 2007.

[BS59] N.N. Bogolyubov and D. V. Shirkov. Introduction to the Theory of Quantized Fields. Interscience Publishers, 1959.

[DDMS10] D.-A. Deckert, D. Dürr, F. Merkl, and M. Schottenloher. Time-evolution of the external field problem in quantum electrodynamics. Journal of Mathematical Physics, 51(12):122301-122328, 2010.

[DG13] J. Dereziński and C. Gérard. Mathematics of Quantization and Quantum Fields. Cambridge University Press, 2013.

[Dim82] J. Dimock. Dirac quantum fields on a manifold. Trans. Amer. Math. Soc., 269:133-147,1982.

[DP07] D. Dürr and P. Pickl. On Adiabatic Pair Creation. Communications in Mathemtical Physics, 282(1):161-198, 2007.

[Dun09] G. V. Dunne. New Strong-Field QED Effects at ELI: Nonperturbative Vacuum Pair Production. European Physical Journal, (D55):327-340, 2009.

[Dys06] F. J. Dyson. Lectures on Advanced Quantum Mechanics Second Edition (1951). arXiv:quant-ph/0608140v1, 2006.

[ELI] http://www.extreme-light-infrastructure.eu/.

[Fin06] F. Finster. The Principle of the Fermionic Projector. American Mathematical Society, 2006.

[FKT12] F. Finster and J. Kleinert and J.-H. Treue. An Introduction to the Fermionic Projector and Causal Fermion Systems Summer School Regensburg. To be published, 2012.

[FS79] H. Fierz and G. Scharf. Particle interpretation for external field problems in QED. Helvetica Physica Acta. Physica Theoretica, 52(4):437-453 (1980), 1979. 
[GHLS13] P. Gravejat, C. Hainzl, M. Lewin, and E. Séré. Construction of the PauliVillars-Regulated Dirac Vacuum in Electromagnetic Fields. Archive for Rational Mechanics and Analysis, 208(2):603-665, 2013.

[Joh82] F. John Partial differential equations. Springer-Verlag, 1982.

[LM96] E. Langmann and J. Mickelsson. Scattering matrix in external field problems. Journal of Mathematical Physics, 37(8):3933-3953, 1996.

[Mic98] J. Mickelsson. Vacuum polarization and the geometric phase: gauge invariance. Journal of Mathematical Physics, 39(2):831-837, 1998.

[RS81] M. Reed and B. Simon. Methods of Modern Mathematical Physics, Vol. 1: Functional Analysis. Academic Press, rev. and enl. edition, 1981.

[Rin09] H. Ringström. The Cauchy problem in general relativity. European Mathematical Society, 2009.

[Rui77a] S. N. M. Ruijsenaars. Charged particles in external fields. I. Classical theory. Journal of Mathematical Physics, 18(4):720-737, 1977.

[Rui77b] S. N. M. Ruijsenaars. Charged particles in external fields. II. The quantized Dirac and Klein-Gordon theories. Communications in Mathematical Physics, 52(3):267-294, 1977.

[Sch48] J. Schwinger. Quantum Electrodynamics. I. A Covariant Formulation. Physical Review, 74:1439, 1948.

[Sch61] S. Schweber. An introduction to relativistic quantum field theory. Dover, 1961.

[SS65] D. Shale and W. F. Stinespring. Spinor representations of infinite orthogonal groups. Journal of Mathematics and Mechanics, 14:315-322, 1965.

[Tay11] M. E. Taylor, Partial differential equations III Springer, 2011.

[Tha92] Thaller, Bernd. The Dirac Equation. Berlin; New York: Springer-Verlag, 1992.

[Tom46] S. Tomonaga. On a relativistically invariant formulation of the quantum theory of wave fields. Progress of Theoretical Physics, 1:27-42, 1946.

[Wal84] R. Wald. General Relativity. University of Chicago Press, 1984. 